2020-08

\title{
Incompressible SPH simulation of flow past horizontal cylinder between plane wall and free surface
}

\author{
Moballa, B
}

http://hdl.handle.net/10026.1/17657

\begin{abstract}
10.1016/j.jfluidstructs.2020.103091
Journal of Fluids and Structures

Elsevier BV
\end{abstract}

All content in PEARL is protected by copyright law. Author manuscripts are made available in accordance with publisher policies. Please cite only the published version using the details provided on the item record or document. In the absence of an open licence (e.g. Creative Commons), permissions for further reuse of content should be sought from the publisher or author. 


\title{
Incompressible SPH simulation of flow past horizontal cylinder between plane wall and free surface
}

\author{
Burniadi Moballa ${ }^{\mathrm{a}}$, Ming-Jyh Chern ${ }^{\mathrm{a}, *}$, AGL Borthwick ${ }^{\mathrm{b}}$ \\ ${ }^{a}$ Department of Mechanical Engineering, National Taiwan University of Science and Technology, \\ Taipei 10607, Taiwan \\ ${ }^{b}$ School of Engineering, The University of Edinburgh, Edinburgh EH9 3JL, UK
}

\begin{abstract}
Flow past a circular cylinder located between free-surface and wall boundaries is modelled numerically using non-uniform particle size incompressible smoothed particle hydrodynamics (SPH). Several enhancements including Rhie and Chow interpolation, colour function-based free surface tracking, and modified particle shifting are introduced to increase the accuracy and efficiency of the method. A parameter study at constant Reynolds number, Re $=150$, examines the influence of Froude number $(\mathrm{Fr}=0.2-0.6)$, submergence ratio $(H / D=0.5-1.0$, where $H$ is the distance between the apex of the cylinder and undisturbed free surface, and $D$ is the cylinder diameter), and bottom gap ratio $(G / D=1.0-5.0$, where $G$ is the distance between the base of the cylinder and the bed) on the ambient free-surface flow pattern and hydrodynamic force on the cylinder. It is found that the vortex shedding pattern depends on all three parameters. As the Froude number increases for fixed submergence ratio and bottom gap ratio, vortex shedding is eventually suppressed. As the submergence and bottom gap ratios increase, the threshold of vortex shedding suppression shifts to higher values of Froude number. The mean drag coefficient depends on the submergence ratio and bottom gap ratio but is independent of Froude number. Meanwhile, the lift coefficient depends on
\end{abstract}

\footnotetext{
${ }^{*}$ Corresponding author

Email address: mjchern@mail.ntust.edu.tw (Ming-Jyh Chern)
} 
submergence ratio and Froude number but is independent of bottom gap ratio. Spectral analysis of force and free-surface elevation time signals shows that the free-surface deformation and lift force are closely related during the vortex shedding regime.

Keywords: incompressible smoothed particle hydrodynamics (ISPH), circular cylinder, free surface, vortex shedding suppression, inlet-outlet boundaries

\section{Introduction}

Flow past a circular cylinder is one of the most closely studied benchmark problems in fluid dynamics due to its relevance to many practical applications such as the design and analysis of submerged pipelines and offshore structures. The basic configuration of flow past a circular cylinder in an infinite medium has been extensively investigated and its fluid dynamics is well understood $[1,2]$. A second configuration that has attracted considerable research interest is the effect of cylinder proximity to a plane wall in otherwise infinite fluid [3, 4]. A third configuration is that of a cylinder located in the vicinity of the free surface; this has been studied experimentally by Sheridan et al. [5] and numerically by Reichl et al. [6], amongst others. The fourth configuration, where the cylinder is placed between a free surface and a plane wall, has however received relatively little attention to date.

Previous studies on the effect of each type of boundary on flow past a cylinder provide useful insight into the flow physics when both types are simultaneously present. Investigations into flow past a circular cylinder near a wall have suggested that the gap between the wall and the cylinder is a particularly important flow control parameter. Bearman and Zdravkovich [7] classified different flow regimes based on the ratio of the gap to the cylinder diameter $(G / D)$. At $G / D=0$, the cylinder impedes the upstream flow and causes the flow to separate from the wall. At small gap 
ratios below the critical value, vortex shedding is suppressed. For gap ratios larger than critical, regular periodic shedding is established. Regular vortex shedding does not occur at very small gap ratios $(0.0<G / D \leq 0.125 D)$ although periodicity is detected in the flow velocity within the outer shear layer [3]. This periodicity can be attributed to the vortex shed from the upper part of the cylinder. At larger gap ratios, $0.25 D \leq G / D \leq 0.375$, the lower wake pairs with the separated wall boundary layer [3]. As a result of this pairing, the wake flows emanating from the lower and upper parts of the cylinder are deflected away from the wall [8]. The pairing also prevents shedding of a vortex into the lower part of the wake. Onset of vortex shedding occurs at $0.5 D \leq G / D \leq 0.75$, with asymmetry observed between the shed vortices from the lower and upper parts of the cylinder $[8,9]$. The shed vortex from the upper part of the cylinder advects nearly parallel to the wall while the shed vortex from the lower part of the cylinder advects away from the wall and eventually crosses over the trajectory of the upper shed vortex. The final relative vertical positions of both vortices are opposite to those in the infinite fluid case $[8,10]$. He et al. [10] found that a secondary vortex also forms, as a consequence of the roll-up of the separated wall boundary layer. At $G / D=1.0$, regular vortex shedding is observed. The lower shed vortex advects vertically to about the same elevation as the upper shed vortex. There is no upstream separation. However, the wall boundary layer separates periodically because of its interaction with vortex shedding downstream of the cylinder [10]. Separation leads to the formation of a secondary vortex which elongates [8], forming a vortex sheet near the wall because of interaction between the secondary vortex and the lower shed vortex. At $G / D=2$, the secondary vortex still forms but it no longer interacts directly with the lower shed vortex. At larger values of gap ratio, no secondary vortex develops. Price et al. [3] found that the Strouhal number, $\mathrm{S}=f^{\prime} D / U$, where $f^{\prime}$ is the vortex shedding frequency and $U$ is the amplitude of the incident flow velocity, increases as the gap ratio decreases provided the Reynolds 
number $\operatorname{Re}<2000$ where $\operatorname{Re}=U D / \nu$ and $\nu$ is the kinematic viscosity coefficient. For $\operatorname{Re}>2000$ , the Strouhal number appears to be insensitive to gap ratio. Previous laboratory studies at Re above the threshold value of 2000 also show that the Strouhal number remains constant as the gap ratio decreases until a critical value of $G / D$ is reached $[7,11,12]$. The mean drag coefficient is not affected by the gap ratio until the position of the cylinder interferes with the wall boundary layer $[12,13]$. Provided the gap distance is less than or equal to the wall boundary layer thickness, the mean drag coefficient decreases as the gap decreases further. The mean lift coefficient decreases with increasing gap ratio up to $G / D<1.5$. When $G / D \geq 1.5$, the mean lift coefficient is approximately zero [12]. All the aforementioned studies consider flow past a cylinder in fluid of infinite extent in the horizontal plane, and so do not consider the effect of gravitational acceleration. For a cylinder in fluid of finite extent and experiencing gravity, the mean lift and drag coefficients both decrease considerably as the gap increases. The effect of fluid depth becomes insignificant when the ratio of fluid depth to cylinder diameter is larger than 4.5 [14]. The root-mean-square lift coefficient, which indicates the strength of vortex shedding, is also affected by the gap ratio. The root-mean-square lift coefficient remains approximately constant for gap ratios below a critical value, and increases sharply when the gap ratio increases above the critical value up to $(G / D \approx 1)$. However, there is hardly any change, when the gap is increased further [12].

Proximity of the cylinder to a free surface in the presence of gravitational acceleration gives rise to quite different flow behaviour. Laboratory tests by Sheridan et al. [5] showed that the ratio between submergence depth and cylinder diameter $(H / D)$ plays an important role. At zero submergence ratio, a shear layer forms on the lower side of the cylinder, similar to that on the upper side of the cylinder in near-wall flow. At small submergence ratio, a jet-like flow structure forms at the top of the cylinder, is directed downward at an angle, and mixes with the shear layer 
emanating from the bottom of the cylinder. As the submergence ratio increases, the mixing point moves progressively downstream. At an intermediate submergence ratio $(H / D=0.75)$, the jet-like structure is attached to the free surface. The angle of the jet is also affected by the Froude number $(\mathrm{Fr}=U / \sqrt{g D})$; the larger the value of Froude number, the more the jet is pulled towards the base of the cylinder. Moreover, the angle of the jet also undergoes periodic oscillation. A numerical study at low Reynolds number $(R e=180)$ undertaken by Reichl et al. [6] confirmed these results. In particular, they found that the oscillation observed in previous laboratory tests [5] also occurred, this time at a much lower Reynolds number. Reichl et al.'s numerical results also demonstrated that the near wake and free-surface fluid motion were affected by the submergence ratio and Froude number. For a small but constant value of submergence ratio, increasing Froude number heightens the severity of free-surface deformation and even leads to local wave breaking. As the free surface deforms, it produces vorticity whose strength increases with Froude number. At a certain threshold Froude number, the strength of the free-surface vorticity is sufficient to suppress vortex shedding in the near wake. As cylinder submergence increases, the threshold of vortex shedding suppression occurs at a higher Froude number. At constant Froude number, the Strouhal number increases with increasing submergence ratio until $H / D \approx 1$; for $H / D>1$, the Strouhal number decreases slightly with increasing submergence ratio. For constant submergence ratio, S decreases with increasing Fr, provided $H / D<1$; however, S increases slightly with increasing Fr, for $H / D \geq 1$. Changes to Fr do not affect $\mathrm{S}$, for $H / D \geq 3[6]$.

The present study aims to examine the effect of the presence of both a solid wall and a liquid free surface on the hydrodynamics of flow past a circular cylinder using incompressible SPH (which is capable of simulating non-linear free-surface flows and wave-breaking processes, and can provide stable, accurate pressure prediction near boundaries $[21,22,23,24])$. In practice, it is usually dif- 
ficult to estimate the properties of the wave generated by the interaction of the free-surface flow with the cylinder, which include the wave height and length. This is where a Lagrangian method, such as incompressible SPH whose free-surface resolving capability does not heavily depend on grid size, offers an advantage. Furthermore, the incompressible SPH method enforces incompressibility theoretically, therefore yields more accurate pressure prediction and avoids the density-error problem in a non-uniform particle setting. Herein, a divergence-free formulation [25] with particle shifting is chosen instead of the constant-density formulation [26]. A number of enhancements are employed, including an inlet-outlet boundary algorithm, non-uniform particle size, and modified free-surface tracking and particle shifting method suitable for non-uniform particles. An adaptation of Rhie and Chow [27] interpolation is incorporated in the SPH method to remedy the checkerboard problem. To validate the model, flow past circular cylinder close to a free surface is simulated. A parameter study is conducted to investigate the effect of submergence ratio, gap ratio, and Froude number, at constant Reynolds number, on vortex shedding from the cylinder, the hydrodynamic force experienced by the cylinder, and motions of the free surface.

Application of the SPH method to flow past a circular cylinder has usually taken place in tandem with the development of an open boundary condition algorithm for SPH. In practice, flow past a circular cylinder placed in an infinite domain has often been employed as a benchmark case in the development of SPH algorithms $[15,16,17]$. A parameter study focusing on the drag coefficient on a circular cylinder for Reynolds number ranging from 1 to 500, using SPH, can be found in [18]. And Bouscasse et al. [19] studied flow past a circular cylinder placed beneath a free surface using the SPH method for the same value of Reynolds number as [6]. The ability of SPH to simulate violent wave breaking allows wider ranges of Froude number and submergence to be considered, while maintaining the same Reynolds number as in [6]. Bouscasse et al. found that vortex shedding reap- 
peared at higher Froude numbers beyond those considered in [6]. They also observed the transition of the free-surface pattern behind the cylinder from flat or regular non-breaking wave conditions to a spilling breaker and finally to a plunging breaker. This work was extended by Colagrossi et al. [20] who found that satisfactory agreement with other numerical methods could be achieved using a particle shifting strategy.

The paper is structured as follows: Section 2 describes the numerical algorithm of the incompressible SPH method utilized herein; Section 3 presents the model setup, results of validation tests and parameter study, and includes flow patterns, hydrodynamic forces, and free-surface elevation dynamics; and Section 4 contains concluding remarks.

\section{Governing Equations and Numerical Scheme}

\subsection{Governing Equations}

In non-dimensional form, the governing equations of incompressible flow may be written as

$$
\nabla \cdot \mathbf{u}=0
$$

and

$$
\frac{\mathrm{D} \mathbf{u}}{\mathrm{D} t}=-\nabla p+\frac{1}{\mathrm{Re}} \nabla^{2} \mathbf{u}+\mathbf{g}
$$

where $\mathbf{u}$ is the flow velocity vector, $\nabla$ is the gradient in the $(x, z)$ plane, $t$ is time, $p$ is pressure, and the vector of gravitational acceleration in the $(x, z)$ plane is $\mathbf{g}=0 \mathbf{i}-\frac{1}{\operatorname{Fr}^{2}} \mathbf{j}$. The Reynolds number and Froude number in Eq. (2) are defined based on the cylinder diameter $D$ as $\operatorname{Re}=\frac{U D}{\nu}$ and $\operatorname{Fr}=\frac{U}{\sqrt{g D}}$, respectively, where $\nu$ is the coefficient of fluid kinematic viscosity, and $U$ is a characteristic flow speed. 


\subsection{SPH Discretizations of the Governing Equations}

The present study employs the corrected SPH gradient discretization proposed by Chen et al. [28] which provides better accuracy, as evidenced by the comparison study by Oger et al. [29]. The Laplacian is discretized using a method proposed by Schwaiger [30]. Applying these SPH discretizations to the right-hand side of the Navier-Stokes momentum equation yields

$$
\frac{\mathrm{D} \mathbf{u}_{i}}{\mathrm{D} t}=-\sum_{j}\left(p_{j}-p_{i}\right)\left(\nabla_{i} W_{i j} \cdot \mathbf{G}_{i}\right) V_{j}+\frac{2}{\operatorname{Re}} L_{i} \sum_{j}\left(\mathbf{u}_{j}-\mathbf{u}_{i}\right)\left(\frac{\mathbf{x}_{i j}}{\left|\mathbf{x}_{i j}\right|^{2}}-\sum_{j} \nabla_{i} W_{i j} V_{j}\right) \cdot \nabla_{i} W_{i j} V_{j}+\mathbf{g}
$$

where $i$ and $j$ are indices representing a particle and its neighbour, $\mathbf{x}_{i j}=\mathbf{x}_{j}-\mathbf{x}_{i}$ is the relative position of a neighbour to a particle, $\nabla_{i} W_{i j}$ is the kernel gradient following differentiation with respect to particle $i$, and $V_{j}$ is the non-dimensional particle volume. The correction matrix for the first gradient [28], $\mathbf{G}_{i}$, is defined as

$$
\mathbf{G}_{i}=\left[\sum_{j} \mathbf{x}_{i j} \otimes \nabla_{i} W_{i j} V_{j}\right]^{-1}
$$

The correction of the Laplacian, $L_{i}$, is defined as the trace of $\mathbf{G}_{i}$ [30],

$$
L_{i}=\operatorname{Tr}\left(\mathbf{G}_{i}\right)
$$

The quintic Wendland function is employed as the kernel function throughout this study.

\subsection{Boundary Conditions}

The wall boundary is modelled using boundary and dummy particles (as suggested by Lee et al. [31], and earlier by Shao and Lo [26]). A number of particles are distributed at equidistant spacing along the boundary line. For each boundary particle, several dummy particles are placed along the boundary's outward normal direction. The number of dummy particles is determined 
such that full kernel support can be maintained. Fig. 1 illustrates the configuration. Information on the connection between a dummy particle and its associated boundary particle is stored, and later employed when applying the boundary condition. A detailed explanation of the methods for enforcing essential boundary conditions is given in [31].

At the outlet, the standard procedure in computational fluid dynamics is to enforce zero velocity and pressure gradients in the normal direction. To achieve this, a buffer zone of length equal to the diameter of the smoothing area is established just beyond the outlet. Any particle that moves beyond the outlet line into the buffer zone then becomes an outlet particle. The velocity of an outlet particle is determined by temporarily dragging the particle horizontally to the outlet line and applying the SPH interpolation formula over a semi-circular support region in the inner flow domain (see Fig. 1), similar to the method proposed by Kazemi et al. [32]. The pressure of an outlet particle is determined by solving the pressure-Poisson equation or enforcing the free-surface boundary condition if the particle lies at the free surface.

In order to set the inlet boundary condition, the particle melting technique is adopted [33]. Initially, a number of inlet boundary particles and their corresponding dummy particles are distributed along the inlet boundary. As an inlet boundary particle enters the domain, it changes into a fluid particle and the dummy particle that follows immediately behind becomes a new inlet boundary particle (see Fig. 1). When solving the pressure-Poisson equation, each inlet boundary particle is treated in the same way as a wall boundary particle. Once the pressure of an inlet boundary particle has been evaluated, the result is copied to the corresponding dummy particles.

Lastiwka et al. [34] proposed a way of maintaining mass conservation that involved creating particles at the inlet and destroying particles that move beyond the outlet, and was implemented by allocating and deallocating memory dynamically. However, this method is not suitable for 
parallel implementation on graphical processing units (GPUs), and so the following approach is adopted herein. An outlet particle that moves beyond the outlet buffer zone is dragged back to the inlet and changed into an inlet dummy particle. The new inlet dummy particle is then associated with the inlet boundary particle that has the smallest number of dummy particles, and/or the smallest normal distance to the inlet boundary line (see Fig. 1). As a consequence, the number of the dummy particles associated with each inlet boundary particle is several times larger than that of dummy particles at the wall because the inlet dummy particles also act as a reserve to maintain mass conservation. This algorithm is similar to the recycling open boundary introduced by Fu and $\operatorname{Jin}[35]$.

\subsection{Free-Surface Particle Tracking}

Unlike the weakly compressible SPH method, the free-surface boundary condition is enforced explicitly in the incompressible SPH method. This explicit enforcement is based on free-surface particle tracking. Some proposals for free-surface particle tracking employ criteria based on the summation of the kernel function [36] or position divergence [31]. A more robust detection method has been proposed by Dilts [37] which uses neighbour particle detection. However, the method is computationally expensive as it requires $N^{2} \log (N)$ operations. Recently, Marrone et al. [38] developed a two-step free-surface tracking method which reduces the computational complexity. The first step screens particles located near the free surface using a criterion based on the minimum eigenvalue of the correction matrix of the kernel gradient $\left(\mathbf{G}_{i}\right)$. In the second step, neighbour scanning over a sector-like section to the normal direction of the free surface is carried out for

the foregoing near-free-surface particles. Although the method has been successfully applied to SPH simulation with uniformly sized particles, it is not suitable for non-uniform particles. The 
kernel is a function of smoothing length, and so the eigenvalue of the correction matrix is also a function of smoothing length. When non-uniform particle sizes are employed in a simulation, the smoothing length will vary according to particle size. Therefore, it is difficult to establish a single threshold value for distinguishing whether or not a particle is located near the surface. Moreover, the threshold value also depends on the type of kernel function adopted. In the present study, the first step of Marrone et al.'s method is modified for non-uniform particles. To avoid dependency on smoothing length and kernel function, a colour function is defined to determine whether a particle lies at the free surface,

$$
c_{i}= \begin{cases}0 & \text { if particle } i \text { is located at the free surface } \\ 1 & \text { otherwise }\end{cases}
$$

As a consequence of Eq. (6), the gradient of the colour function of a particle located near the free surface is non-zero. This is applied as a criterion in the first step of the free-surface tracking algorithm. The second step consists of scanning for the presence of any neighbouring particle(s) within a sector-like section in the normal direction to the free surface (similar to [38]). The steps of the algorithm are as follows:

1. Calculate the gradient of the colour function,

$$
\nabla c_{i}=\sum_{j}\left(c_{j}-c_{i}\right) \nabla_{i} W_{i j} \cdot \mathbf{G}_{i} V_{j} .
$$

2. If $\left|\nabla c_{i}\right|=0, c_{i}$ remains the same.

3. If $\left|\nabla c_{i}\right| \neq 0$, calculate the direction normal to the free surface and perform a neighbour scan [38]. If one or more particle(s) is detected inside the scan region, set $c_{i}=1$ and vice versa. 
When $\left|\nabla c_{i}\right| \neq 0$, the unit normal vector to the free surface is calculated as

$$
\mathbf{n}=-\frac{\nabla c_{i}}{\left|\nabla c_{i}\right|}
$$

\subsection{Timestepping}

The present study combines the time integration method of the projection SPH method [25] and the implicit incremental projection method for solving the pressure-velocity coupling. The resulting numerical procedure over a single time step is described below:

1. Move the particle to an intermediate position,

$$
\mathbf{x}_{i}^{*}=\mathbf{x}_{i}^{n}+\Delta t \mathbf{u}_{i}^{n}
$$

2. Predict the velocity,

$$
\mathbf{u}_{i}^{*}=\mathbf{u}_{i}^{n}+\Delta t\left(-\nabla p_{i}^{n}+\frac{1}{\operatorname{Re}} \nabla^{2} \mathbf{u}_{i}^{*}+\mathbf{g}\right)
$$

3. Obtain the pressure correction by solving the pressure-Poisson equation,

$$
\nabla^{2} \delta p_{i}=\frac{\nabla \cdot \mathbf{u}_{i}^{*}}{\Delta t}
$$

4. Correct the velocity and the pressure,

$$
\begin{gathered}
\mathbf{u}_{i}^{n+1}=\mathbf{u}_{i}^{*}-\Delta t \nabla \delta p_{i}, \\
p_{i}^{n+1}=p_{i}^{n}+\delta p_{i} .
\end{gathered}
$$

5. Move the particle to the new position,

$$
\mathbf{x}_{i}^{n+1}=\mathbf{x}_{i}^{n}+\Delta t \frac{\mathbf{u}_{i}^{n+1}+\mathbf{u}_{i}^{n}}{2}
$$




\subsection{Rhie and Chow Interpolation for ISPH Method}

The particle-based discretization adopted by the SPH method defines both the velocity vector and pressure scalar at the same location, similar to a collocated grid-based method. The so-called checkerboard problem which afflicts collocated grid-based methods, also occurs in the SPH method (The checkerboard problem is a spatial oscillation in pressure which appears as zigzag patterns in the pressure contours.). In the collocated grid-based method, the checkerboard problem can be remedied by applying the Rhie and Chow [27] interpolation method. Generally, the formula of Rhie and Chow interpolation can be written as

$$
\tilde{\mathbf{u}}=\overline{\mathbf{u}}_{f}-\Delta t\left(\nabla_{n} p_{f}-\left.\overline{\nabla_{n} p}\right|_{f}\right)
$$

where $\tilde{\mathbf{u}}$ is the interpolated velocity at the interface between cells, $\overline{\mathbf{u}}_{f}$ is the distance-weighted average velocity at the cell interface, $\nabla_{n} p_{f}$ is the normal pressure gradient at the cell interface, and $\left.\overline{\nabla_{n} p}\right|_{f}$ is the distance-weighted average pressure gradient at the interface. $\overline{\mathbf{u}}_{f}$ and $\left.\overline{\nabla_{n} p}\right|_{f}$ are averaged between the cell of interest and the neighbouring cell. If the interface is located at the midpoint between cell centres, Eq. (15) can be simplified into

$$
\tilde{\mathbf{u}}=\frac{\mathbf{u}_{i}+\mathbf{u}_{j}}{2}-\Delta t\left(\nabla_{n} p_{f}-\frac{\nabla_{n f} p_{i}+\nabla_{n f} p_{j}}{2}\right)
$$

where $i$ and $j$ denote the present cell and its neighbour, respectively, and subscript $n f$ stands for the normal direction to the interface. Eq. (16) can be applied in the SPH method by taking the particles to be cells and assuming that the interface between adjacent particles is located at the midpoint of the inter-particle distance. The pressure gradient at the interface of particle $i$ and $j$ can be calculated as

$$
\nabla_{n} p_{f}=\frac{p_{j}-p_{i}}{\left|\Delta \mathbf{x}_{i j}\right|} \mathbf{n}_{i j}
$$


where $\mathbf{n}_{\mathbf{i j}}$ is the unit normal vector at the particle interface obtained from the relative position vector by

$$
\mathbf{n}_{i j}=\frac{\mathbf{x}_{i j}}{\left|\mathbf{x}_{i j}\right|}=n_{x, i j} \mathbf{i}+n_{y, i j} \mathbf{j}=\frac{x_{j}-x_{i}}{\left|\mathbf{x}_{i j}\right|} \mathbf{i}+\frac{y_{j}-y_{i}}{\left|\mathbf{x}_{i j}\right|} \mathbf{j}
$$

The pressure gradient of particle $i$ to the normal direction of the interface with neighbouring particle $j$ can be obtained from

$$
\nabla_{n f} p_{i}=\frac{\partial p_{i}}{\partial n_{i j}} \mathbf{n}_{i j}
$$

where

$$
\frac{\partial p_{i}}{\partial n_{i j}}=\nabla p_{i} \cdot \mathbf{n}_{i j}=n_{x, i j} \frac{\partial p_{i}}{\partial x}+n_{y, i j} \frac{\partial p_{i}}{\partial y}
$$

The pressure gradient of particle $j$ to the normal direction of the interface with particle $i$ can be calculated similarly. Substituting Eqs. (17)-(19) to Eq. (16) yields

$$
\tilde{\mathbf{u}}_{j}=\frac{\mathbf{u}_{i}+\mathbf{u}_{j}}{2}-\Delta t\left(\frac{p_{j}-p_{i}}{\left|\Delta \mathbf{x}_{i j}\right|}-\frac{\frac{\partial p_{i}}{\partial n_{i j}}+\frac{\partial p_{j}}{\partial n_{i j}}}{2}\right) \mathbf{n}_{i j}
$$

When the interface velocity is used to calculate the velocity divergence, the support length becomes effectively half of the original support length owing to the definition of the interface at the midpoint of the inter-particle distance. Therefore, the formula of the SPH velocity divergence becomes

$$
\nabla \cdot \mathbf{u}_{i}=\sum_{j}\left(\tilde{\mathbf{u}}_{j}-\mathbf{u}_{i}\right) \cdot\left(\nabla_{i} W_{i j}\left(\frac{h}{2}, \frac{\Delta \mathbf{x}_{i j}}{2}\right) \mathbf{G}_{i}\left(\frac{h}{2}, \frac{\Delta \mathbf{x}_{i j}}{2}\right)\right) V_{j} .
$$

Substituting Eq. (21) into Eq. (22) yields

$\nabla \cdot \mathbf{u}_{i}=\sum_{j}\left[\frac{\mathbf{u}_{i}+\mathbf{u}_{j}}{2}-\Delta t\left(\frac{p_{j}-p_{i}}{\left|\Delta \mathbf{x}_{i j}\right|} \mathbf{n}_{i j}-\frac{\frac{\partial p_{i}}{\partial n_{i j}}+\frac{\partial p_{j}}{\partial n_{i j}}}{2} \mathbf{n}_{i j}\right)-\mathbf{u}_{i}\right] \cdot\left[\nabla_{i} W_{i j}\left(\frac{h}{2}, \frac{\Delta \mathbf{x}_{i j}}{2}\right) \mathbf{G}_{i}\left(\frac{h}{2}, \frac{\Delta \mathbf{x}_{i j}}{2}\right)\right] V_{j}$

Recognizing that $\nabla_{i} W_{i j}\left(\frac{h}{2}, \frac{\Delta \mathbf{x}_{i j}}{2}\right)=2 \nabla_{i} W_{i j}\left(h, \Delta \mathbf{x}_{i j}\right)$ and $\mathbf{G}_{i}\left(\frac{h}{2}, \frac{\Delta \mathbf{x}_{i j}}{2}\right)=\mathbf{G}_{i}\left(h, \Delta \mathbf{x}_{i j}\right)$, Eq. 
can be simplified and rearranged into

$$
\nabla \cdot \mathbf{u}_{i}=\sum_{j}\left(\tilde{\mathbf{u}}_{j}-\mathbf{u}_{i}\right) \cdot\left(\nabla_{i} W_{i j} \mathbf{G}_{i}\right) V_{j}
$$

where $\tilde{\mathbf{u}}_{j}$ is the interpolated velocity redefined as

$$
\tilde{\mathbf{u}}_{j}=\mathbf{u}_{j}-\Delta t\left[2 \frac{p_{j}-p_{i}}{\left|\Delta \mathbf{x}_{i j}\right|}-\left(\frac{\partial p_{i}}{\partial n_{i j}}+\frac{\partial p_{j}}{\partial n_{i j}}\right)\right] \mathbf{n}_{i j} .
$$

Therefore, each component of the interpolated velocity can be calculated as

$$
\begin{aligned}
& \tilde{u}_{j}=u_{j}-\Delta t\left[2 \frac{p_{j}-p_{i}}{\left|\mathbf{x}_{i j}\right|}-\left(\frac{\partial p_{i}}{\partial n_{i j}}+\frac{\partial p_{j}}{\partial n_{i j}}\right)\right] n_{x, i j}, \text { and } \\
& \tilde{v}_{j}=v_{j}-\Delta t\left[2 \frac{p_{j}-p_{i}}{\left|\mathbf{x}_{i j}\right|}-\left(\frac{\partial p_{i}}{\partial n_{i j}}+\frac{\partial p_{j}}{\partial n_{i j}}\right)\right] n_{y, i j} .
\end{aligned}
$$

Eq. (24) is later used in the pressure-Poisson equation, Eq. (11).

\subsection{Wave Absorption}

We now turn to the location of the cylinder in the numerical tank with respect to the upstream and downstream boundaries. Due to the deformability of the liquid free surface and the presence of an obstacle, it is expected that reflected waves may develop upstream and/or downstream of the cylinder. A numerical treatment is required to prevent wave reflection at an open boundary causing interference with the interior solution. This task is accomplished by deploying a numerical wave absorption layer immediately after the inlet and before the outlet; the damping model is similar to that of Mayer et al. [39]. At the inlet, the wave absorption layer is applied solely to the velocity. The absorption formula is denoted as

$$
\mathbf{u}=\mathbf{u}(1-\gamma)+\gamma \mathbf{u}_{\text {inlet }}
$$

where $\mathbf{u}_{\text {inlet }}$ is the prescribed inlet velocity at the same elevation as $\mathbf{u}$ and $\gamma$ is the relaxation parameter [40] calculated by,

$$
\gamma=1-\frac{\exp x_{r}^{\mu}-1}{\exp 1-1}, x_{r}=\frac{x-x_{\text {start }}}{x_{\text {start }}-x_{\text {end }}}
$$


where $\mu=3.5, x$ is the coordinate of the particle, $x_{\text {start }}$ is the start of the absorption layer, and $x_{e n d}$ is the end of the absorption layer. Consequently, any disturbance to the free-surface level upstream of the cylinder is transmitted to the inlet and the free-surface level at the inlet is adjusted accordingly by adding or removing the inlet particle layer [41]. In practice, the adjustment is carried out when the disturbance enters the inlet wave absorption layer. If the free-surface level increase is equal or larger than the initial particle spacing, a further particle layer is added, and vice versa. The velocities of the particles in the new layer are calculated using the same damping model. Meanwhile, the damping model is solely applied to the free-surface level at the outlet wave absorption layer in order to enforce the constant free-surface level condition. Therefore, the elevations of free-surface particles inside the outlet wave absorption layer are adjusted according to the damping model,

$$
\zeta=\zeta(1-\gamma)+\gamma \zeta_{\text {outlet }}
$$

\subsection{Particle Refinement and De-refinement}

It is beneficial to use a non-uniform particle size distribution in order to reduce the computational load because of the long domain required. This is achieved by applying zone-based dynamic refinement and de-refinement similar to [42] with slight modification. In essence, when a particle enters a zone which contains smaller-sized particles, the entry particle is split into three child particles similar to the triangular arrangement in $[42,43]$. The mass of each child particle after refinement is one-third that of the parent particle. Fig. 2 illustrates the configuration of child particles during the refinement process. The child particles are distributed along a circle which is centred at the position of the parent particle. The diameter of the circle is given by,

$$
d_{a r}=\frac{\left|\Delta \mathbf{x}_{0, b r}\right|}{\sqrt{3}}
$$


where $\Delta \mathbf{x}_{0}$ is the initial spacing of the parent particle, and the subscripts ar and $b r$ stand for "after refinement" and "before refinement", respectively. Smoothing lengths of the child particles after refinement are also calculated in a similar fashion using Eq. (30) where $\left|\Delta \mathbf{x}_{0, b r}\right|$ is replaced with the smoothing length of the parent particle. In the azimuthal direction, child particles are distributed at an equidistant spacing of $120^{\circ}$. The first child particle is placed at an angle of $60^{\circ}$ to the nearest neighbouring particle (see Fig. 2) to avoid particle clustering. Velocities and pressures of the particles after refinement are determined using SPH interpolation with the normalized kernel, giving

$$
\begin{aligned}
\mathbf{u}_{i, a r} & =\sum_{j} \mathbf{u}_{j} \frac{W_{i j} V_{j}}{\sum_{j} W_{i j} V_{j}}, \text { and } \\
p_{i, a r} & =\sum_{j} p_{j} \frac{W_{i j} V_{j}}{\sum_{j} W_{i j} V_{j}} .
\end{aligned}
$$

The de-refinement process combines a particle with two of its nearest same-size neighbours. The position of the new particle after de-refinement is at the centroid of the parent particles. The mass of the child particle after de-refinement is the sum of the mass of the parent particles, while the smoothing length is $\sqrt{3}$ times the smoothing length of a parent particle. Field variables of a child particle in the de-refinement process are obtained using the same method as in the refinement process.

Many works on particle refinement in the weakly compressible SPH method initialize the velocity of a child particle by enforcing momentum and energy conservation between parent and child particles during splitting and coalescing, besides minimizing the density error, i.e. [15, 42, 44, 45]. The present study uses a slightly different approach by employing an interpolation technique. Despite the fact that such an approach is uncommon in SPH, this interpolation method has been widely adopted to initialize child cells in adaptive mesh refinement. An adaptation of this tech- 
nique in weakly compressible SPH can be found in a study by Chiron et al. [46] who discovered that the mass variation incurred by interpolation method is only about $0.2 \%$. Earlier application of an SPH interpolation formula for initializing properties of a child particle can be found in [47]. While density-error minimization is important in a weakly-compressible setting where density is allowed to vary, it is rather irrelevant in divergence-free incompressible SPH where density is held constant. Discrete conservation of momentum in an incompressible setting can be maintained by enforcing the divergence-free velocity field [48]. This is further elaborated in 3.2 Validation and Particle Independence.

\subsection{Modified Particle Shifting}

The incompressible SPH method can suffer from tensile instability which causes particle clustering that leads the algorithm to diverge. $\mathrm{Hu}$ and Adams [49] found that this problem is related to the term on the right-hand side of the pressure-Poisson equation. If a divergence-free condition is solely enforced in the SPH method, then the constant density condition is violated. As a result, several modifications have been proposed for application to the right-hand side of the pressurePoisson equation, such as varying the particle density $[49,50]$. However, the pressure predicted by this method is less accurate than that obtained from the pressure-Poisson equation with velocity divergence on the right-hand side. A combination of both velocity divergence and variation of particle density is proposed later to overcome this problem $[51,52]$. Comparison of the performance of these three formulations can be found in [53]. Xu et al. [54] introduced an alternative approach which consists of a re-distribution strategy based on shifting the particle position and subsequently adjusting the field variables using Taylor series. Particle shifting prevents particle clustering and maintains a uniform particle distribution. However, application of this method is limited to inter- 
nal flow and dam-break like flow problems because of the truncated kernel support near the free surface. To overcome this limitation, a new re-meshing algorithm based on Fick's diffusion equation has been proposed by Lind et al. [55] and enhanced by Skillen et al. [56].

A modification to the particle shifting algorithm is proposed herein aimed at handling freesurface flows involving open boundaries and non-uniform particle sizes. Work in this area has also been undertaken by Vacondio et al. [15]. The steps of the original algorithm which consist of shifting particle position followed by correction of the field variables remain the same but the method used to calculate the shift distance is modified, taking into account both the non-uniform particle size and the liquid free-surface. To account for the non-uniformity of particle size, volume-weighted averaging and summation are used to calculate the average neighbour distance and shift vector, respectively. The modification is designed so that in the limit of uniform particle size, the formula converges to the original method in [54]. Near the free surface where the support area of a particle is truncated, the shifting algorithm tends to produce a shift vector directed to the outward normaldirection of the free surface which eventually causes the particle motions to blow up. To solve this problem, layers of ghost particles are placed along the outward normal of the free surface from each free-surface particle. The technique resembles the space potential particle method [57]. The distance of a ghost particle is the same as the initial vertical spacing of the fluid particles and the number of layers is the same as the number of layers of dummy particles at the wall boundary. A ghost particle is considered to be an element of the neighbour set solely during particle shifting. The following special treatment is applied to a free-surface particle, similar to the approach taken in [58]. If the normal component of the shift vector is directed outward, then solely the tangential direction is used in the calculation of the shift distance. The shift coefficient of a free-surface particle is $0.25-0.5$ of the shift coefficient of an inner particle. The detailed steps of the modified shifting 
algorithm are as follows:

1. Calculate the average distance to the neighbouring particles using particle-volume weighted averaging,

$$
r_{i, a v g}=\frac{\sum_{j}\left|\mathbf{x}_{i j}\right| V_{j}}{\sum_{j} V_{j}}
$$

2. Calculate the shift direction vector,

$$
\mathbf{R}_{i}=\sum_{j} \frac{r_{i, a v g}^{2}}{\left|\mathbf{x}_{i j}\right|^{2}} \frac{\mathbf{x}_{i j}}{\left|\mathbf{x}_{i j}\right|} \frac{V_{j}}{V_{i}}
$$

3. Determine the shift distance vector as,

$$
\delta \mathbf{r}_{i}= \begin{cases}C_{f s} U_{\infty} \Delta t \mathbf{R}_{t, i} & \text { if particle } i \text { lies at the free surface, } \\ C U_{\infty} \Delta t \mathbf{R}_{i} & \text { otherwise }\end{cases}
$$

where $C=0.01-0.1$ is the shift coefficient, $C_{f s}=0.25 C-0.5 C$ is the shift coefficient of the free-surface particle, $U_{\infty}$ is the reference velocity, and subscript $t$ denotes the tangential component of the shift distance vector.

4. Shift the position of the particle,

$$
\mathbf{x}_{i}^{\prime}=\mathbf{x}_{i}+\delta \mathbf{r}_{i}
$$

and correct the field variables,

$$
\begin{aligned}
& \mathbf{u}_{i}^{\prime}=\mathbf{u}_{i}+\nabla \mathbf{u}_{i} \cdot \delta \mathbf{r}_{i}, \\
& p_{i}^{\prime}=p_{i}+\nabla p_{i} \cdot \delta \mathbf{r}_{i} .
\end{aligned}
$$

While the general steps of the present modified shifting algorithm is similar to those of [15], there are notable differences. First, Vacondio et al.'s method employs ordinary averaging to calculate the average distance whereas the present scheme uses particle volume-weighted averaging that accounts 
for particle size differences. Second, the shift vector summation in [15] is weighted by the ratio of the neighbouring particle mass to the total mass of neighbouring particles, whereas the present method employs the volume ratio of the neighbouring particle to the current particle of interest.

\subsection{Parallel Implementation}

The present numerical scheme is implemented on a GPU (graphics processing unit) using the CUDA programming environment. Parallelization is carried out by assigning one or more threads to each particle. All real-valued variables are defined using double-precision floating-point arithmetic. Fig. 3 presents the flow chart covering a single time-step of the algorithm. Each process located on the GPU consists of one or more CUDA kernels. Generally, the steps are similar to those in [59], except for some modifications. The code is compiled using gcc and nvcc compilers, and the resulting program is run under a Linux operating system. The present study employs two GPUs: NVIDIA Tesla C2075 and NVIDIA Tesla K20C. Both implementations use the fixed-radius nearestneighbour search algorithm to construct the interaction list. The first step consists of creating a grid of coarse cells whose widths are equal to the smoothing length of the largest particle followed by grouping the particles into the cells according to their positions. Cell membership data are stored using a format similar to Compressed Row Storage (CRS), whereas a cell-linked list is employed in [59]. Each cell is treated as the row of a matrix, and corresponding particles located in the cell are treated as non-zero entries in CRS format. Therefore, only row-pointer and column-index arrays are required to store the data. The row-pointer array marks the location of the particle indexes of each cell in the column-index array which stores the indexes of consecutive particles that belong to each cell. Constructions of the row-pointer and column-index arrays are carried out in the GPU using the parallel prefix-sum algorithm [60]. 
Although the resulting code could achieve 6-7 times speedup compared to the OpenMP parallelized CPU code, the performance is nevertheless bounded by latency because of the mesh-free nature of the SPH method. The parallel execution model of a CUDA program requires parallel memory access. However, parallel memory access can only be undertaken at certain successive locations in the memory. If the requested data value is located outside those consecutive addresses, the access is serialized. This memory coalescing constraint cannot always be satisfied because the particles are located randomly, although uniformly distributed. This condition becomes worse over time. Initially, data concerning two neighbouring particles are located near each other in the memory. The distance between two neighbouring particles can grow larger than the smoothing length because of the movements of both particles, at which point they no longer remain neighbours. However, their respective data are still located near each other in the memory. Moreover, each particle can gain a new neighbour for which data are located far from those of the other neighbours. Therefore, the number of data requests increases and, consequently, the execution time lengthens. To prevent this, after the nearest-neighbour search algorithm is performed, the particles are re-indexed according to their position in the cell. A new index is assigned to each particle such that particles in the same cell have consecutive indices. Following the re-indexing step, the data are moved to a new location in the array according to the new index. This step is called particle re-ordering in [59]. The re-indexing process is implemented also using the parallel prefix-sum algorithm. After the particle is re-indexed, the neighbour list data of each particle is constructed from the grid. The same CRS storage is employed where the particle is regarded as a matrix row, and the column-index array is filled with the indexes of the neighbour particles. Only neighbours located inside the smoothing circle are enlisted in the array to save computational resources. This array is also constructed on the GPU using the same parallel prefix-sum algorithm. Finally, the neighbour list of each particle 
is sorted in ascending order to maintain the memory-access pattern.

For a CUDA kernel that performs gradient calculation, or other workloads, which involve summation over neighbouring particles, a two-dimensional thread layout is employed. In the present GPU, four threads are assigned to each particle, provided the threads are members of the same warp; the loop work over neighbouring particles is divided among those threads. Therefore, the thread block consists of $64 \times 4$ threads. An array of $64 \times 4$ shared variables is defined to store the temporary summation result from each thread. The final result of the calculation is obtained using a reduction operation among the assigned threads. This method is illustrated in Fig. 4.

In the present numerical method, the predicted velocity $\mathbf{u}^{*}$ is obtained implicitly by solving the discrete parabolic equation (Eq. (10)). Therefore, with the addition of the discrete PPE, the algorithm solves a linear system three times, which necessitates that construction of the coefficient matrices is also performed in the GPU. Coefficient matrices are stored in CRS format, and so, this task is accomplished again using the parallel prefix-sum algorithm. First, a particle is flagged as 1 if it belongs to the coefficient matrix and 0 if it does not. By summing the flags, the total number of rows and diagonal entries is obtained. Next, the number of off-diagonal entries for each row is determined; and incrementing the number of off-diagonal entries by 1 yields the total number of non-zero entries in the row. The number of non-zero entries of each row is stored in a separate array. The row-pointer array is constructed by applying parallel prefix-sum to the number of nonzero entries. Diagonal and off-diagonal entries of the coefficient matrix are filled using two different CUDA kernels. A further CUDA kernel is used to sum the dummy particle contributions at the boundary. The BiCGStab method is implemented by performing each operation in separate CUDA kernels. Dot-product and matrix-vector multiplications are performed using functions supplied by the optimized CUBLAS library, while the SAXPY operations are handled using an in-house 
developed CUDA kernel. Error calculation and error bound checks are performed in the CPU, due to the fact that they are single arithmetic operations and determine the termination of the linear solver. Refinement and de-refinement operations are performed in the CPU. Both operations are sequential by nature, and the number of particles involved is small compared to the total number

of particles. As a consequence, at the end of each time step, particle data necessary to perform refinement and de-refinement are transferred from GPU to CPU. After the operations, new states of the particles involved in those operations are transferred back to the GPU. For the same reasons, the mass conservation algorithm is also implemented in the CPU using a similar strategy.

\section{Results and Discussion}

\subsection{Model Setup}

Fig. 5 depicts the flow configuration. A velocity profile according to Blasius' solution is imposed in the inlet. At the outlet, a Neumann boundary condition is applied to the velocity. At the bottom wall, a non-slip boundary condition is prescribed. At the free surface, constant pressure is applied. To obtain a unique solution, the free-surface elevation at the outlet is kept constant, whereas the free-surface elevation at the inlet is allowed to change. The cylinder is placed $25 D$ from the inlet and the outlet is located $30 D$ from the cylinder. Numerical wave absorption zones are placed immediately after the inlet and before the outlet to damp any wave radiated from the cylinder. The greyscaled rectangles in Fig. 5 represent the refinement zones utilized in the present study. Three different particle sizes are employed. The innermost zone has particle spacing of $\Delta x / D=0.018$ for the parameter study. This spacing has been determined according to a particle independence study, described in the next subsection. Particle spacing in the outer zones is calculated using Eq. $(20)$. 


\subsection{Validation and Particle Independence}

First, we simulate flow past a circular cylinder in an infinite medium at $\operatorname{Re}=34$ and 40 . At these Reynolds numbers, the benchmark case admits a steady-state solution where two symmetric vortices form behind the cylinder. Fig. 6 shows the horizontal-velocity profiles in the vertical direction at $x=2$, along with previous experimental results [61,62]. Fig. 7 compares the pressure coefficient along the surface of the cylinder at $R e=40$ predicted by the present scheme with experimental data obtained by Grove et al. [63]. The agreement between the numerical predictions and previous experimental data in Figs. 6 and 7 confirms the satisfactory performance of the present pressure-solver. Discrepancies observed in both figures are attributed to the inability of the present two-dimensional simulation to model three-dimensional effects in the experiments.

Flow past a circular cylinder close to a free surface at $\operatorname{Re}=180, \mathrm{Fr}=0.3$, and $H / D=1.0$ has also been simulated to validate the present numerical scheme. Table 1 lists the mean hydrodynamic force predicted for different particle spacing against the corresponding results from alternative numerical predictions by [64] who used a finite volume solver with a body fitted grid. In general, the present numerical predictions are in good agreement with Reichl's computations. Judging from the relative difference which is less than $3 \%$ in this case, an initial particle spacing of $\Delta x / D=0.018$ is adequate for the subsequent parameter study. Fig. 8 compares free-surface profiles at the peak and trough of the lift force time series predicted by the present numerical scheme with the results in [6]. However, this is not a precise comparison because the lift-force time-series experienced some limit-cycle modulation and we did not have information on the exact sampling time of the free-surface data in [6]. Moreover, Reichl et al. employed a two-fluid model with active second-fluid (air) and relaxed density ratio in contrast to the present single-fluid model which implies passive air. Nevertheless, the agreement between both set of results validates the present free-surface detection 
algorithm against a well-established numerical alternative.

Fig. 9 shows different coloured free-surface and near free-surface particles superimposed on the pressure contours in the vicinity of particle size transition boundaries. No particle gaps occur near the particle size transition boundaries. Pressure contours are smooth in the transition regions, confirming the satisfactory performance of the free surface detection method in a non-uniform particle setting. Fig. 10 demonstrates the ability of the method to track the highly-deformed freesurface and the air pocket created during wave breaking. Moreover, the velocity profiles in the transition regions before and after the cylinder are also smooth, as seen in Fig. 11. The smoothness of pressure contours in Fig. 9 is an indication that any errors generated by the particle refinement and de-refinement processes do not have any significant effect. Moreover, the velocity profiles in the transition regions before and after the cylinder are also smooth, as seen in Fig. 11. This smoothness can be attributed to the correction step of the incremental pressure projection method employed in the present study. In an incompressible SPH setting, any error generated by splitting or combining particles manifests itself as velocity divergence or a mass residual. The velocity divergence is then minimized by the pressure and velocity correction step (steps 3 and 4 in 2.5 Timestepping). The particle distributions and smoothness of pressure contours and velocity profiles in the vicinity of the refinement boundaries confirm that the modified particle-shifting algorithm functioned properly. Incorrect particle shifting close to the free surface would generate numerical error from the inability of the smaller particles to match their larger size neighbours due to the shorter smoothing length. Fig. 12 illustrates the performance of the present shifting algorithm in the limit of uniform particle size by comparing particle distributions in the vicinity of the cylinder with and without shifting.

The checkerboard problem can be alleviated using SPH interpolation carried out during the post-processing phase of an SPH simulation. Moreover, in SPH benchmark cases where gravity is 
the main driving force of the flow, and the hydrostatic component dominates the pressure field, pressure oscillation caused by checkerboarding does not considerably affect the results. However, a more accurate pressure estimate is required in the present work which examines the interaction between hydrodynamic forces around the cylinder and the free surface dynamics. Fig. 13 compares the pressure field with and without Rhie and Chow interpolation. A checkerboard pattern can be seen around the cylinder in the left-hand figure, obtained without Rhie and Chow interpolation. Smooth pressure contours are observed around the cylinder in the right-hand figure, obtained using Rhie and Chow interpolation. This demonstrates that the checkerboard effect is filtered out by the interpolation herein.

\subsection{Parameter Study}

A parameter study is now carried out to investigate the effect of the free surface and bottom wall boundaries on the hydrodynamics of flow past a circular cylinder, at relatively low Reynolds number. Here the submergence ratio $(H / D)$, the bottom gap ratio $(G / D)$, and the Froude number are varied while the Reynolds number is kept constant. The values of the parameters are chosen such that $\mathrm{Re}=$ $150 ; \mathrm{Fr}=0.2,0.3,0.4,0.5$, and $0.6 ; H / D=0.5,0.75$, and 1.0 ; and $G / D=1.0,2.0,3.0$, and 5.0. It should be noted that the submergence $(H)$ is defined as the vertical distance from the apex of the cylinder to the still water level and the bottom gap $(G)$ is defined as the vertical distance from the base of the cylinder to the wall boundary.

\subsubsection{Flow Patterns}

Fig. 14 shows the instantaneous free-surface profiles and underlying vorticity distributions at $t=200$ at increasing values of Froude number, when $G / D=1.0$ and $H / D=0.5$. At $\mathrm{Fr}=$ 0.2 , asymmetric vortex shedding occurs behind the cylinder where the upper shed vortex evolves 
downstream to become considerably larger than the lower shed vortex. Due to confinement by the free surface and the bottom boundary, both the upper and lower shed vortices follow the same path as they advect downstream. The upper shed vortex develops a circular shape with a slightly flattened top. Meanwhile, the lower shed vortex elongates and deforms into a rounded triangular shape, and gradually dissipates as it advects downstream. The vortex shedding process also induces periodic separation of the bed boundary layer. As it moves downstream, the upper vortex gradually mixes with the separated bed boundary layer which eventually re-attaches to the bottom boundary. Initially, two counter-rotating vortices are shed, the upper one of negative (i.e. clockwise) vorticity. In this case, the flow pattern is classified as $-\mathrm{S}(2 \mathrm{~S})$ using the notation proposed in [65]. At Fr $=0.2$, there is only minor deformation of the free surface, with no substantial production of free-surface vorticity. At $\mathrm{Fr}=0.3$, the free surface starts to generate a considerable amount of vorticity within a zone up to $5 D$ downstream of the cylinder. When the Froude number increases to 0.4, the freesurface deformation produces a vortex that advects downwards and coalesces with the lower shed vortex. Therefore, the lower vortex is able to travel further downstream before becoming dissipated. At higher Fr $=0.5-0.6$, the strength of the free-surface vorticity is sufficient to stabilize the near wake and entirely suppress vortex shedding. In the vortex shedding suppression regime, denoted here as $\mathrm{N}$, the attached vortex pair is asymmetric and the upper vortex is larger than the lower vortex. The degree of asymmetry is inversely proportional to the strength of the free-surface vortex. As the free-surface vortex grows larger, the vortex attached to the upper part of the cylinder shrinks and, consequently, the degree of asymmetry decreases.

The flow pattern at larger submergence is similar to $H / D=0.5$ described above. Nevertheless, the stabilizing effect of the free-surface vorticity weakens and the degree of asymmetry between the upper and lower shed vortices becomes more pronounced as the submergence increases. At 
$H / D=0.75$, although the flow pattern is similar to that of $H / D=0.5$ for $\mathrm{Fr}=0.2-0.4$, the effect of the weakening of free-surface vorticity appears in the vortex shedding suppression region for $\mathrm{Fr}=0.5-0.6$ where the upper attached vortex becomes considerably longer compared to $H / D=0.5$. Fig. 15 depicts the corresponding free-surface profiles and vorticity distributions for submergence ratio of $H / D=1$. It can be observed that the flow pattern depicted in Fig. 15 is similar to that in Fig. 14 for Fr $=0.2-0.4$ with the strength of the upper shed vortex growing with Froude number. Meanwhile, the lower shed vortex dissipates faster at higher values of submergence ratio. In Fig. 15d, the upper attached vortex is extremely long and becomes unstable due to the further weakening of the effect of free-surface vorticity; here the instability resembles coalesced negative vortices, and so the flow pattern is classed as -C.

The flow pattern for $G / D=2$ evolves in the same manner as $G / D=1$ with increasing Froude number. Although asymmetric vortex shedding occurs at low Fr, vortex shedding becomes suppressed as the Froude number increases. Meanwhile, the asymmetry between upper and lower shed vortices reverses. Fig. 16 shows the flow vorticity pattern for $H / D=0.5$ and $G / D=2$. At all values of Froude number considered, the lower shed vortices are consistently stronger than the upper shed vortices along the length of the domain. At Fr $=0.2-0.3$, the upper shed vortex has sufficient strength to advect itself downstream while mixing with the bottom boundary layer, forming a $2 \mathrm{~S}$ pattern. At Fr $=0.4$, the degree of asymmetry has increased further. The upper shed vortex is smaller than that in the lower Froude number case, and eventually dissipates as it travels downstream, leaving behind solely the lower shed vortex. At Fr $=0.5$ the upper shed vortex no longer mixes with the bottom boundary layer and effectively dissipates while advecting downstream. The flow vorticity patterns at $\mathrm{Fr}=0.4-0.5$ are classed $+\mathrm{S}(2 \mathrm{~S})$ for a similar reason as the corresponding pattern for $G / D=1$. At $\operatorname{Fr}=0.5$, the near wake is stretched downstream and vortex shedding 
commences far from the cylinder. Complete suppression of vortex shedding happens at $\mathrm{Fr}=0.6$.

Fig. 17 shows that the evolved flow patterns at $H / D=0.75$ are quite similar to those at $H / D=0.5$. However, the degree of asymmetry between the upper shed vortex and lower shed vortex is lower compared to $H / D=0.5$, resulting in a $2 \mathrm{~S}$ pattern for $\mathrm{Fr}=0.2-0.5$. Accordingly, mixing between the upper shed vortex and bottom boundary layer can be observed up to $\mathrm{Fr}=0.5$. Incomplete vortex shedding suppression is apparent at $\mathrm{Fr}=0.6$. The attached vortices are elongated downstream with single vortex shedding starting to occur far downstream from the elongated lower attached vortex; here the flow pattern is $+\mathrm{S}$. When the submergence ratio increases to 1 , the strengths of the upper and lower shed vortices are relatively similar (see Fig. 18) and a $2 \mathrm{~S}$ pattern appears for Fr $=0.2-0.6$. Free-surface vorticity is no longer able to stabilize the near wake and, as a result, vortex shedding still takes place at the highest Froude number considered.

Fig. 19 shows a snapshot of the free-surface flow and vorticity contours for $H / D=0.5$ and $G / D=3$ at different Fr values ranging from 0.2 to 0.6 . In general, the patterns are similar to those obtained for the same submergence, but at $G / D=2$. However, the degree of asymmetry between upper and lower shed vortices is higher for $G / D=3$ than $G / D=2$. At $\mathrm{Fr}=0.2-0.3$, a $2 \mathrm{~S}$ pattern appears, while mixing occurs between fluid in the upper shed vortex and bottom boundary layer. The $2 \mathrm{~S}$ pattern changes into $+\mathrm{S}(2 \mathrm{~S})$ as Fr increases to $0.4-0.5$. As it advects, the upper shed vortex loses strength and dissipates about $5 D$ downstream of the cylinder; the far wake is composed of a single street of anticlockwise, elliptically shaped vortices. Similar to the results for Fr $=0.6$ at $H / D=0.75$ and $G / D=2$, incomplete suppression of vortex shedding appears to occur at $\mathrm{Fr}=0.6$. The lower attached vortex is not completely stabilized, and the resulting vorticity contour comprises coalesced vortices (similar to the pattern for $G / D=1, H / D=1$, and $\mathrm{Fr}=0.5$ ); in this case, the flow pattern is classed $+\mathrm{C}$. 
Similar to the results for $G / D=2$, asymmetry between the upper and lower shed vortices decreases with increasing submergence at constant Froude number for a gap ratio $G / D=3$; this is caused by the increasingly large strength of the upper shed vortex. Consequently, it can be seen from Fig. 20 that the upper and lower shed vortices have relatively similar strength at lower Froude numbers $(\mathrm{Fr}=0.2-0.4)$ for $H / D=0.75$, leading to development of a $2 \mathrm{~S}$ vortex shedding pattern. Substantial asymmetry begins to arise between upper and lower shed vortices at $\mathrm{Fr}=0.5$; this leads to the $+\mathrm{S}(2 \mathrm{~S})$ pattern becoming established at $\mathrm{Fr}=0.6$ instead of vortex shedding suppression. When the submergence ratio increases to 1 , the single-vortex street also disappears and a $2 \mathrm{~S}$ pattern appears (at all Fr considered here).

Fig. 21 shows the results obtained for $H / D=0.5$ and $G / D=5$. For $F r=0.2-0.4$, the flow pattern is classed as $+\mathrm{S}(2 \mathrm{~S})$, noting the asymmetry between the upper and lower shed vortices. The upper shed vortex is considerably weaker compared to its lower counterpart, and disappears as it migrates downstream, mainly due to suppression by the free surface. This phenomenon becomes more pronounced, the higher the value of Fr. The lower shed vortices elongate to become ellipsoidal as they propagate downstream. With increasing Froude number, the vortex straining process grows in severity and the lower shed vortex becomes sufficiently long and thin that successive lower shed vortices start to coalesce downstream at $\mathrm{Fr}=0.5$. The resulting vorticity pattern is $+\mathrm{C}(2 \mathrm{~S})$. At $\mathrm{Fr}=0.6$, the near wake stretches further downstream and the upper attached vortex is stabilized by the free-surface vorticity. However, because the gap ratio is large and there is no direct interaction with the bottom boundary, we can still observe vortex shedding from the lower part of the cylinder which again develops into a single-vortex street corresponding to $+\mathrm{S}$ pattern.

For gap ratio $G / D>3$, asymmetry between upper and lower shed vortices decreases with increasing submergence ratio. Fig. 22 shows that a $2 \mathrm{~S}$ pattern occurs for $\mathrm{Fr}=0.2-0.4$ and a 
$+\mathrm{S}(2 \mathrm{~S})$ pattern occurs for $\mathrm{Fr}=0.5-0.6$ at $H / D=0.75$ and $G / D=5$ (which closely resembles the corresponding results for $H / D=0.75$ and $G / D=3$ ). Similar to $G / D=2-3$, a 2 S pattern for $G / D=5$ appears at all Fr considered, when the submergence ratio increases to 1 .

The deformed free surface also changes with increasing Fr at fixed submergence and gap ratios. Fig. 23 shows the free-surface wave profile and the underlying vorticity distribution for $H / D=0.5$ and $G / D=2$, corresponding to a typical wave breaking case. At a low Froude number of $\mathrm{Fr}=0.2$, small-amplitude regular waveforms develop behind the cylinder. The wave height increases with increasing Froude number (see Fig. 23b). At Fr $=0.4$, plunging wave breakers occur periodically according to the vortex shedding frequency; each plunging breaker produces free-surface vorticity causing a further vortex to form, which advects downward before the onset of the next plunging breaker. The free-surface vortex finally merges with the lower shed vortex, increasing the asymmetry between upper and lower shed vortices. At $\mathrm{Fr}=0.5$, the breaking wave comprises a periodic collapsing breaker with an associated vortex attached to the free surface. At Fr $=0.6$, a steady overturning wave is evident similar to a hydraulic jump. The front roller merges with the upper attached vortex and suppresses vortex shedding. The behaviour of the deforming free surface with increasing Froude number is similar to results presented by Reichl et al. [6].

The following discussion summarizes the above findings for vorticity in the flow past a cylinder located in the vicinity of the free surface and a flat bed at a relatively low Reynolds number. Upper and lower vortices shed from a horizontal cylinder become asymmetric when upper free surface and lower bed boundaries are present. At fixed gap and submergence ratios, the asymmetry becomes more pronounced as Fr increases until a threshold is reached above which vortex shedding is suppressed. This matches the description by Reichl et al. [6] for a cylinder in a semi-infinite, free-surface flow with no bed boundary. As also observed by Reichl et al., suppression of vortex 
shedding commences at higher Fr as the submergence ratio $H / D$ increases, at constant $G / D$. Increasing the bottom gap weakens the tendency to suppress vortex shedding and gives rise to an unstable attached vortex pair implying that vortex shedding suppression occurs at higher Fr as $G / D$ increases. This transformation of the vortex shedding behaviour with increasing $G / D, H / D$, and Fr suggests that there exists a Froude number which marks the onset of the transition leading to vortex shedding suppression and is proportional to the submergence and gap ratios. Fig. 24 presents a map, summarizing the flow patterns obtained over the parameter ranges considered.

\subsubsection{Hydrodynamic Force and Free-Surface Elevation}

Fig. 25 presents the mean drag coefficient as a function of Froude number for submergence ratios $H / D=0.5,0.75$ and 1 for four gap ratios $G / D=1,2,3$, and 5 . For any given pair of values of submergence ratio and gap ratio, the mean drag coefficient appears almost independent of Fr. For a given Froude number and gap ratio, the mean drag coefficient increases with decreasing submergence. Comparison between Figs. 25a-d indicates that the mean drag coefficient decreases with increasing gap ratio for a given submergence; this is in contrast with results by Lei et al. [12] and Zdravkovich [13] who found that the average drag coefficient is not affected by gap ratio when the cylinder is placed above the wall boundary layer in semi-infinite fluid in the horizontal plane. However, the present finding does match that of by Chiew [14] who considered flow past a cylinder in liquid of finite depth in the presence of gravity. Figs. 25a-d indicate that discrepancies in mean drag coefficient between $H / D=0.5,0.75$, and 1 become smaller as $G / D$ increases. The mean drag coefficient curves for the three different submergence ratios almost coincide for gap ratio of $G / D=5$. These findings imply that the effect of submergence on mean drag coefficient becomes less pronounced with increasing bottom gap ratio. 
Fig. 26 shows the mean lift coefficient as a function of Fr obtained for all submergence and gap ratios. The body force component results in a negative mean lift coefficient, and so the absolute value is displayed in Fig. 26 for convenience. Here the magnitude of mean lift coefficient increases with increasing Fr for a given submergence. Similar to the mean drag coefficient, the magnitude of the mean lift coefficient also increases with decreasing submergence. By comparing Figs. 26a-d, it is interesting to see that the mean lift coefficient is only slightly affected by increasing gap ratio. This finding, again, is similar to [14] but different to $[12,13]$ (who found that the mean lift coefficient is affected by the gap ratio). For constant Fr, the discrepancies in mean lift coefficient for the three submergence ratios only slightly change with increasing gap ratio; this suggests that the effect of submergence is independent of bottom gap ratio.

A better indicator for the strength of vortex shedding and the onset of its suppression is given by the amplitude of the fundamental frequency of lift force, as depicted in Fig. 27. At $G / D=1$, the amplitude of lift force decreases rapidly with increasing Fr for a given submergence ratio up to $\mathrm{Fr}=0.4$, in accordance with the weakening of vortex shedding. The amplitude of lift force at $G / D=1$ is plotted for $\mathrm{Fr}=0.2-0.4$ only, because the spectral analysis failed to extract any meaningful frequency from the time domain data at $\mathrm{Fr}=0.5$ and 0.6 , in keeping with vortex shedding suppression (see Figs. 14 and 15). Similar behaviour can also be seen for Fr $=0.5-0.6$ at $G / D=2, \operatorname{Fr}=0.5$ at $G / D=3$, and $\operatorname{Fr}=0.5$ at $G / D=5$, in each case where vortex shedding suppression also occurs. For $G / D=2$ and 3, the amplitude of lift force increases as Fr approaches 0.3 followed by a rapid decrease as Fr increases further, at a given submergence ratio. Commencement of the rapid decrease of amplitude shifts to $\mathrm{Fr}=0.4$ for $G / D=5$. The slope and relative position of the curves in the region of rapid decrease confirm the present supposition that the threshold Froude number which marks the onset of transition to vortex shedding suppression 
increases with increasing submergence and gap ratios.

The amplitude of lift force remains almost constant as Fr increases from 0.2 to 0.4 for $G / D=5$ which suggests that the strength of vortex shedding is not affected by the Froude number (Fig. 27). For $G / D=1-3$ and $\mathrm{Fr}=0.2-0.3$, the lift force amplitude increases as the submergence ratio increases from 0.5 to 0.75 and then slightly decreases as the submergence ratio increases to 1 , at constant Fr. Meanwhile, the amplitude of the lift force progressively increases with increasing submergence for $\operatorname{Fr} \geq 0.4$. This phenomenon disappears at $G / D=5$; the amplitude of lift force consistently increases with increasing submergence. However, the amplitudes of lift force for $H / D=$ 0.75 and 1 are very similar, suggesting that the effect of submergence only becomes noticeable when $H / D<0.75$.

Fig. 28 presents the predominant frequencies within the lift force and free-surface elevation time series (determined through spectral analysis) as functions of Froude number for fixed values of submergence and gap ratios. The frequency of the lift force appears to be almost independent of Froude number, within the range considered. This result is slightly different to [6] who found that the Strouhal number decreases slightly with increasing Froude number. For $G / D=1-2$, the lift force frequency increases with increasing submergence for constant Fr, in accordance with [6] for infinitely deep free-surface flow past a horizontal cylinder. The lift force frequency decreases with increasing bottom gap ratio, in keeping with results obtained in [3]. Discrepancies in lift force frequency for $H / D=0.5,0.75$, and 1 decrease with increasing bottom gap ratio. For $G / D=3$ and 5 , the lift force frequencies for difference submergence ratios almost coincide; this behaviour is quite similar to that of the mean drag coefficient. It is interesting to note that the dominant frequency in the free-surface elevation time history invariably coincides with the lift force frequency for all data points in Fig. 28 which implies that the lift force is closely related to the free-surface wave. 


\section{Conclusions}

An incompressible SPH model is developed, capable of simulating viscous free-surface flow involving inlet and outlet boundaries with particles of non-uniform size. Validation and particle independence tests show that the model predicts the velocity profiles, pressure coefficient, freesurface contours, and hydrodynamic force in agreement with previously published results obtained using experimental and alternative numerical methods. A parameter study has been carried out to investigate flow past a horizontal, circular cylinder placed between liquid free surface and solid wall boundaries at constant Reynolds number, $\mathrm{Re}=150$. The study reveals that the free-surface flow physics in the vicinity of the cylinder depends on Froude number (Fr, in the range $0.2-0.5)$, submergence ratio $(H / D$, in the range $0.5-1)$ and bottom gap ratio $(G / D$, in the range $1-5)$. At fixed values of submergence ratio and bottom gap ratio, the flow field alters from vortex shedding to a suppressed regime with increasing Froude number; this is also indicated by the amplitude of the lift coefficient which decreases as the Froude number rises. Transition to vortex shedding suppression occurs at higher Froude number when either the submergence ratio or bottom gap ratio increases. The free-surface deformation pattern alters from regular waves at low Froude number to a plunging wave breaker, then a collapsing breaker, and finally a steady overturning wave, as the Froude number increases. The mean drag coefficient decreases with increasing submergence and bottom gap ratios but is independent of Froude number. In contrast, the mean lift coefficient is affected by submergence ratio and Froude number, but is only slightly affected by the bottom gap ratio. The effect of submergence on the mean drag coefficient becomes less pronounced with increasing gap ratio, unlike the effect of submergence on the mean lift coefficient which is not affected by the gap ratio. Lift and free-surface elevation frequencies decrease with increasing submergence 
and gap ratios. Similar to the mean drag coefficient, the effect of submergence on the lift force and free-surface elevation frequencies becomes less pronounced with increasing gap ratio. Lift and freesurface elevation frequencies are consistently close to each other over the entire parameter range, which suggests close coupling between the free-surface wave deformation and the lift force on the cylinder.

\section{Acknowledgements}

The authors would like to express their gratitude to the Ministry of Science and Technology, Taiwan for financial support through grant 107-2221-E-011-075-MY3.

\section{References}

[1] Williamson, C.H.K.. Vortex dynamics in the cylinder wake. Ann Rev Fluid Mech 1996;28:477539.

[2] Zdravkovich, M.M.. Flow Around Circular Cylinders Vol 1: Fundamentals. New York: Oxford University Press; 1997.

[3] Price, S.J., Sumner, D., Smith, J.G., Leong, K., Païdoussis, M.P.. Flow visualization around a circular cylinder near to a plane wall. J Fluids Struct 2002;16(2):175-191.

[4] Sumer, B.M., Fredsøe, J.. Hydrodynamics around cylindrical structures. Singapore: World Scientific; 2006.

[5] Sheridan, J., Lin, J.C., Rockwell, D.. Flow past a cylinder close to a free surface. J Fluid Mech 1997;330:1-30. 
[6] Reichl, P., Hourigan, K., Thompson, M.. Flow past a cylinder close to a free surface. J Fluid Mech 2005;533:269-296.

[7] Bearman, P.W., Zdravkovich, M.M.. Flow around a circular cylinder near a plane boundary. J Fluid Mech 1978;89(1):33-47.

[8] Sarkar, S., Sarkar, S.. Large-Eddy Simulation of Wake and Boundary Layer Interactions Behind a Circular Cylinder. J Fluids Eng 2009;131(9):091201.

[9] Dipankar, A., Sengupta, T.. Flow past a circular cylinder in the vicinity of a plane wall. J Fluids Struct 2005;20(3):403-423.

[10] He, G.S., Wang, J.J., Pan, C., Feng, L.H., Gao, Q., Rinoshika, A.. Vortex dynamics for flow over a circular cylinder in proximity to a wall. J Fluid Mech 2017;812:698-720.

[11] Buresti, G., Lanciotti, A.. Mean and fluctuating forces on a circular cylinder in cross-flow near a plane surface. J Wind Eng Ind Aerodyn 1992;41(1-3):639-650.

[12] Lei, C., Cheng, L., Kavanagh, K.. Re-examination of the effect of a plane boundary on force and vortex shedding of a circular cylinder. J Wind Eng Ind Aerodyn 1999;80(3):263-286.

[13] Zdravkovich, M.M.. Forces on a circular cylinder near a plane wall. Appl Ocean Res $1985 ; 7(4): 197-201$.

[14] Chiew, Y.. Flow Around Horizontal Circular Cylinder in Shallow Flows. J Waterw Port, Coastal, Ocean Eng 1991;117(2):120-135.

[15] Vacondio, R., Rogers, B.D., Stansby, P.K., Mignosa, P., Feldman, J.. Variable resolution 
for SPH : A dynamic particle coalescing and splitting scheme. Comput Methods Appl Mech Eng 2013;256:132-148.

[16] Marrone, S., Colagrossi, a., Antuono, M., Colicchio, G., Graziani, G.. An accurate SPH modeling of viscous flows around bodies at low and moderate Reynolds numbers. J Comput Phys 2013;245:456-475.

[17] Sefid, M., Fatehi, R., Shamsoddini, R.. A Modified Smoothed Particle Hydrodynamics Scheme to Model the Stationary and Moving Boundary Problems for Newtonian Fluid Flows. J Fluids Eng 2016;137(3):031201.

[18] Gholami, M., Galindo-torres, S.A., Scheuermann, A., Williams, D.J.. Parametric study on smoothed particle hydrodynamics for accurate determination of drag coefficient for a circular cylinder. Water Sci Eng 2017;10(2):143-153.

[19] Bouscasse, B., Colagrossi, A., Marrone, S., Souto-Iglesias, A.. SPH modelling of viscous flow past a circular cylinder interacting with a free surface. Comput Fluids 2017;146:190-212.

[20] Colagrossi, A., Nikolov, G., Durante, D., Marrone, S., Souto-Iglesias, A.. Viscous flow past a cylinder close to a free surface: Benchmarks with steady, periodic and metastable responses, solved by meshfree and mesh-based schemes. Comput Fluids 2019;181:345-363.

[21] Shao, S., Ji, C., Graham, D.I., Reeve, D.E., James, P.W., Chadwick, A.J.. Simulation of wave overtopping by an incompressible SPH model. Coast Eng 2006;53(9):723-735.

[22] Ataie-Ashtiani, B., Shobeyri, G., Farhadi, L.. Modified incompressible SPH method for simulating free surface problems. Fluid Dyn Res 2008;40(9):637-661. 
[23] Gotoh, H., Khayyer, A., Ikari, H., Arikawa, T., Shimosako, K.. On enhancement of Incompressible SPH method for simulation of violent sloshing flows. Appl Ocean Res 2014;46:104-115.

[24] Liang, D., Jian, W., Shao, S., Chen, R., Yang, K.. Incompressible SPH simulation of solitary wave interaction with movable seawalls. J Fluids Struct 2017;69(September 2016):72-88.

[25] Cummins, S.J., Rudman, M.. An SPH Projection Method. J Comput Phys 1999;152(2):584607.

[26] Shao, S., Lo, E.Y.M.. Incompressible SPH method for simulating Newtonian and nonNewtonian flows with a free surface. Adv Water Resour 2003;26(7):787-800.

[27] Rhie, C.M., Chow, W.L.. Numerical study of the turbulent flow past an airfoil with trailing edge separation. AIAA J 2008;21(11):1525-1532.

[28] Chen, J.K., Beraun, J.E., Carney, T.C.. A corrective smoothed particle method for boundary value problems in heat conduction. Int J Numer Methods Eng 1999;46(2):231-252.

[29] Oger, G., Doring, M., Alessandrini, B., Ferrant, P.. An improved SPH method: Towards higher order convergence. J Comput Phys 2007;225(2):1472-1492.

[30] Schwaiger, H.F.. An implicit corrected SPH formulation for thermal diffusion with linear free surface boundary conditions. Int J Numer Methods Eng 2008;75(6):647-671.

[31] Lee, E.S., Moulinec, C., Xu, R., Violeau, D.. Comparisons of weakly compressible and truly incompressible algorithms for the SPH mesh free particle method. J Comput Phys 2008;227(18):8417-8436. 
[32] Kazemi, E., Nichols, A., Tait, S., Shao, S.. SPH modelling of depth-limited turbulent open channel flows over rough boundaries. Int J Numer Methods Fluids 2017;83(1):3-27.

[33] Tan, S.K., Cheng, N.S., Xie, Y., Shao, S.. Incompressible SPH simulation of open channel flow over smooth bed. J Hydro-Environment Res 2015;9(3):340-353.

[34] Lastiwka, M., Basa, M., Quinlan, N.J.. Permeable and non-reflecting boundary conditions in SPH. Int J Numer Methods Fluids 2009;61(7):709-724.

[35] Fu, L., Jin, Y.C.. A mesh-free method boundary condition technique in open channel flow simulation. J Hydraul Res 2013;51(2):174-185.

[36] Randles, P.W., Libersky, L.D.. Smoothed Particle Hydrodynamics: Some recent improvements and applications. Comput Methods Appl Mech Eng 1996;139(96):375-408.

[37] Dilts, G.A.. Moving least-squares particle hydrodynamics II: conservation and boundaries. Int J Numer Methods Eng 2000;48(10):1503-1524.

[38] Marrone, S., Colagrossi, A., Le Touzé, D., Graziani, G.. Fast free-surface detection and level-set function definition in SPH solvers. J Comput Phys 2010;229(10):3652-3663.

[39] Mayer, S., Garapon, A., Sørensen, L.S.. A fractional step method for unsteady freesurface flow with applications to non-linear wave dynamics. Int J Numer Methods Fluids 1998;28(2):293-315.

[40] Fuhrman, D.R., Madsen, P.A., Bingham, H.B.. Numerical simulation of lowest-order shortcrested wave instabilities. J Fluid Mech 2006;563:415-441. 
[41] Federico, I., Marrone, S., Colagrossi, A., Aristodemo, F., Antuono, M.. Simulating 2D open-channel flows through an SPH model. Eur J Mech B/Fluids 2012;34:35-46.

[42] Feldman, J., Bonet, J.. Dynamic refinement and boundary contact forces in SPH with applications in fluid flow problems. Int J Numer Methods Eng 2007;72:295-324.

[43] Spreng, F., Schnabel, D., Mueller, A., Eberhard, P.. A local adaptive discretization algorithm for Smoothed Particle Hydrodynamics. Comput Part Mech 2014;1(2):131-145.

[44] Vacondio, R., Rogers, B.D., Stansby, P.K., Mignosa, P.. Shallow water SPH for flooding with dynamic particle coalescing and splitting. Adv Water Resour 2013;58:10-23.

[45] Barcarolo, D.A., Le Touzé, D., Oger, G., De Vuyst, F.. Adaptive particle refinement and derefinement applied to the smoothed particle hydrodynamics method. J Comput Phys 2014;273:640-657.

[46] Chiron, L., Oger, G., de Leffe, M., Le Touzé, D.. Analysis and improvements of Adaptive Particle Refinement (APR) through CPU time, accuracy and robustness considerations. J Comput Phys 2018;354:552-575.

[47] Reyes López, Y., Roose, D., Recarey Morfa, C.. Dynamic particle refinement in SPH: Application to free surface flow and non-cohesive soil simulations. Comput Mech 2013;51(5):731-741.

[48] Martin, D.F., Colella, P., Graves, D.. A cell-centered adaptive projection method for the incompressible Navier-Stokes equations in three dimensions. J Comput Phys 2008;227(3):18631886. 
[49] Hu, X.Y., Adams, N.A.. An incompressible multi-phase SPH method. J Comput Phys 2007;227(1):264-278.

[50] Lo, E.Y.M., Shao, S.. Simulation of near-shore solitary wave mechanics by an incompressible SPH method. Appl Ocean Res 2002;24(5):275-286.

[51] Pozorski, J., Wawrenczuk, A.. SPH computation of incompressible viscous flows. J Theor Appl Mech 2002;40:917-937.

[52] Aly, A.M.. Modeling of multi-phase flows and natural convection in a square cavity using an incompressible smoothed particle hydrodynamics. Int J Numer Methods Heat Fluid Flow 2015;25(3):513-533.

[53] Gui, Q., Dong, P., Shao, S.. Numerical study of PPE source term errors in the incompressible SPH models. Int J Numer Methods Fluids 2015;77(6):358-379.

[54] Xu, R., Stansby, P., Laurence, D.. Accuracy and stability in incompressible SPH (ISPH) based on the projection method and a new approach. J Comput Phys 2009;228(18):6703-6725.

[55] Lind, S.J., Xu, R., Stansby, P.K., Rogers, B.. Incompressible smoothed particle hydrodynamics for free-surface flows: A generalised diffusion-based algorithm for stability and validations for impulsive flows and propagating waves. J Comput Phys 2012;231(4):1499-1523.

[56] Skillen, A., Lind, S., Stansby, P.K., Rogers, B.D.. Incompressible smoothed particle hydrodynamics (SPH) with reduced temporal noise and generalised Fickian smoothing applied to body-water slam and efficient wave-body interaction. Comput Methods Appl Mech Eng 2013;265:163-173. 
[57] Tsuruta, N., Khayyer, A., Gotoh, H.. Space potential particles to enhance the stability of projection-based particle methods. Int J Comut Fluid Dyn 2015;29(1):100-119.

[58] Sun, P.N., Colagrossi, A., Marrone, S., Zhang, A.M.. The $\delta$ plus-SPH model: Simple procedures for a further improvement of the SPH scheme. Comput Methods Appl Mech Eng $2017 ; 315: 25-49$.

[59] Chow, A.D., Rogers, B.D., Lind, S.J., Stansby, P.K.. Incompressible SPH (ISPH) with fast Poisson solver on a GPU. Comput Phys Commun 2018;226:81-103.

[60] Harris, M., Sengupta, S., Owens, J.D.. Parallel prefix sum(scan) with CUDA. In: Nguyen, H., editor. GPU Gems3; chap. 39. Addison Wesley; 2007, p. 851-876.

[61] Kovasznay, L.S.G.. Hot-wire investigation of the wake behind cylinders at low Reynolds numbers. Proc R Soc London Ser A Math Phys Sci 1949;198(1053):174-190.

[62] Nishioka, M., Sato, H.. Measurements of velocity distributions in the wake of a circular cylinder at low Reynolds numbers. J Fluid Mech 1974;65(1):97-112.

[63] Grove, A.S., Shair, F.H., Petersen, E.E., Acrivos, A.. An experimental investigation of the steady separated flow past a circular cylinder. J Fluid Mech 1964;19(1):60-80.

[64] Reichl, P.. Flow past a cylinder close to a free surface. Ph.D. thesis; Monash University; 2001.

[65] Williamson, C.H.K., Roshko, A.. Vortex formation in the wake of an oscillating cylinder. J Fluids Struct 1988;2(4):355-381. 


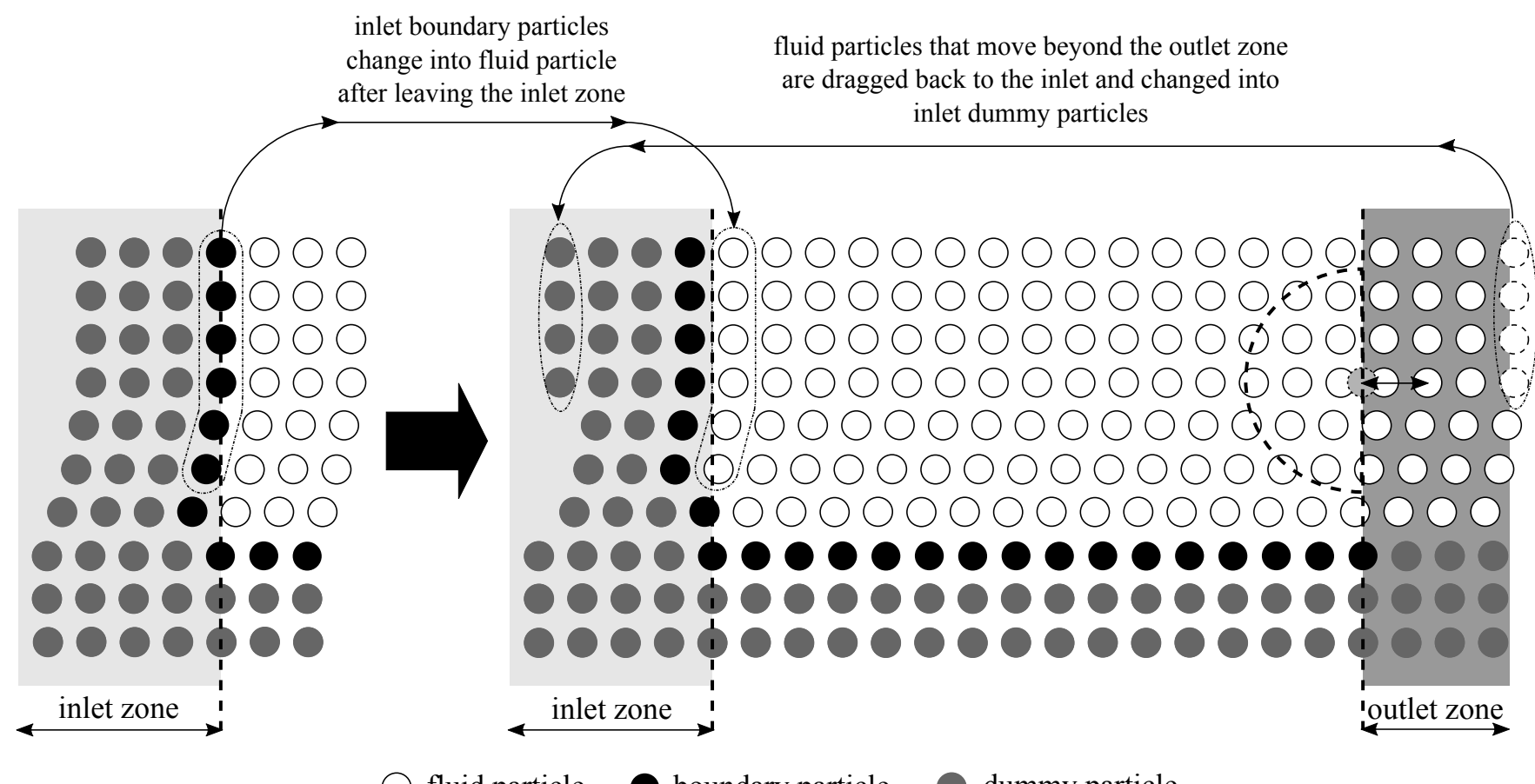

fluid particle boundary particle dummy particle

Fig. 1 Schematic diagram illustrating methods used for enforcing inlet and outlet boundary conditions and maintaining mass conservation.

Table 1 Mean hydrodynamic force on a horizontal cylinder in unidirectional free-surface flow with Re $=180$, $\mathrm{Fr}=0.3$, and $H / D=1.0$ for different particle spacing.

\begin{tabular}{c|c|c|} 
& $C_{L}$ (\% diff. $)$ & $C_{D}$ (\% diff.) \\
\hline Reichl [64] & -0.1242 & 1.54 \\
$\Delta x / D=0.03$ & $-0.1162(6.4 \%)$ & $1.49(3.4 \%)$ \\
$\Delta x / D=0.02$ & $-0.1238(0.3 \%)$ & $1.48(3.8 \%)$ \\
$\Delta x / D=0.018$ & $-0.1243(0.008 \%)$ & $1.50(2.8 \%)$ \\
$\Delta x / D=0.015$ & $-0.1277(2.8 \%)$ & $1.50(2.8 \%)$
\end{tabular}




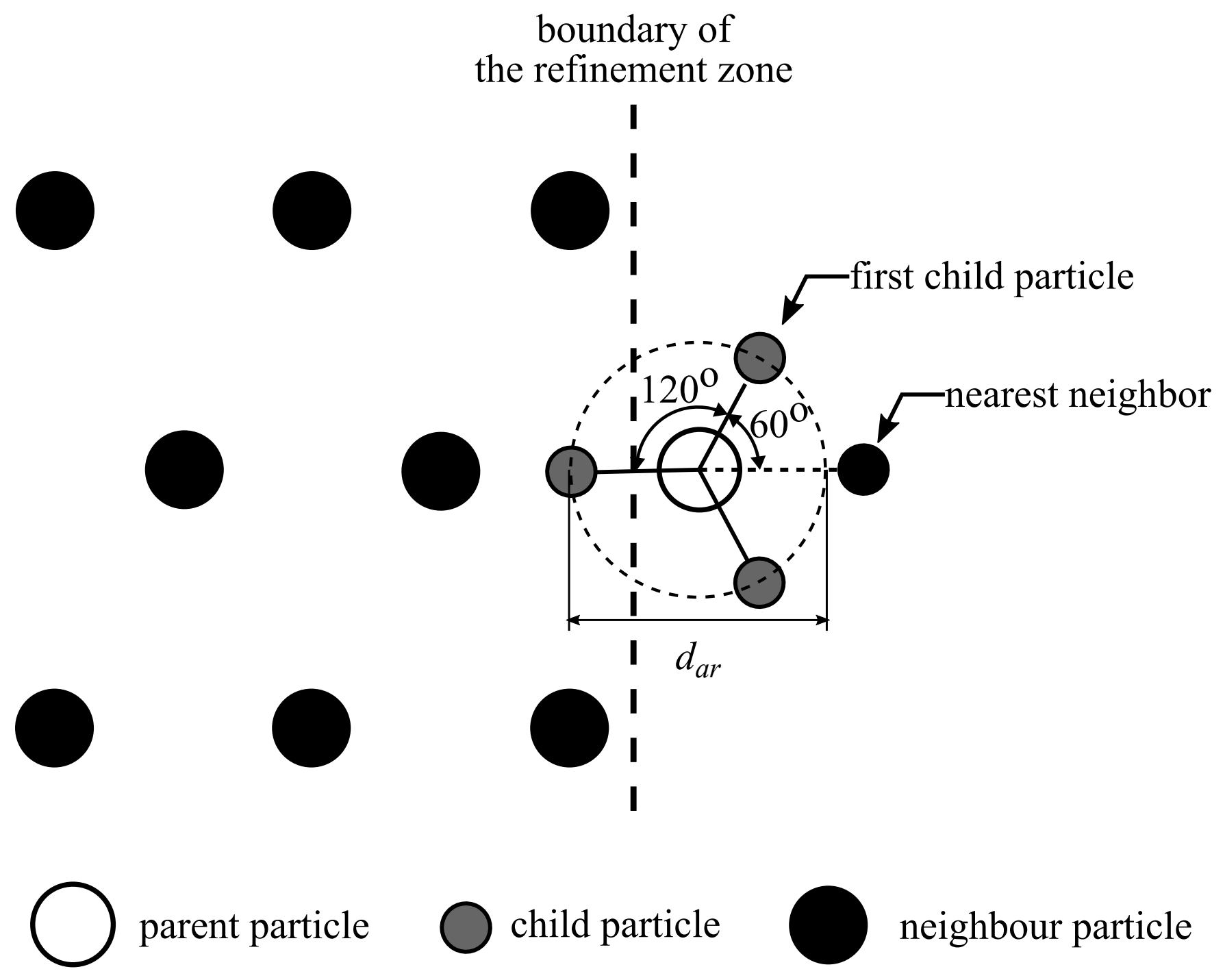

Fig. 2 Distribution and placement of child particles relative to the nearest neighbour during the refinement process. 


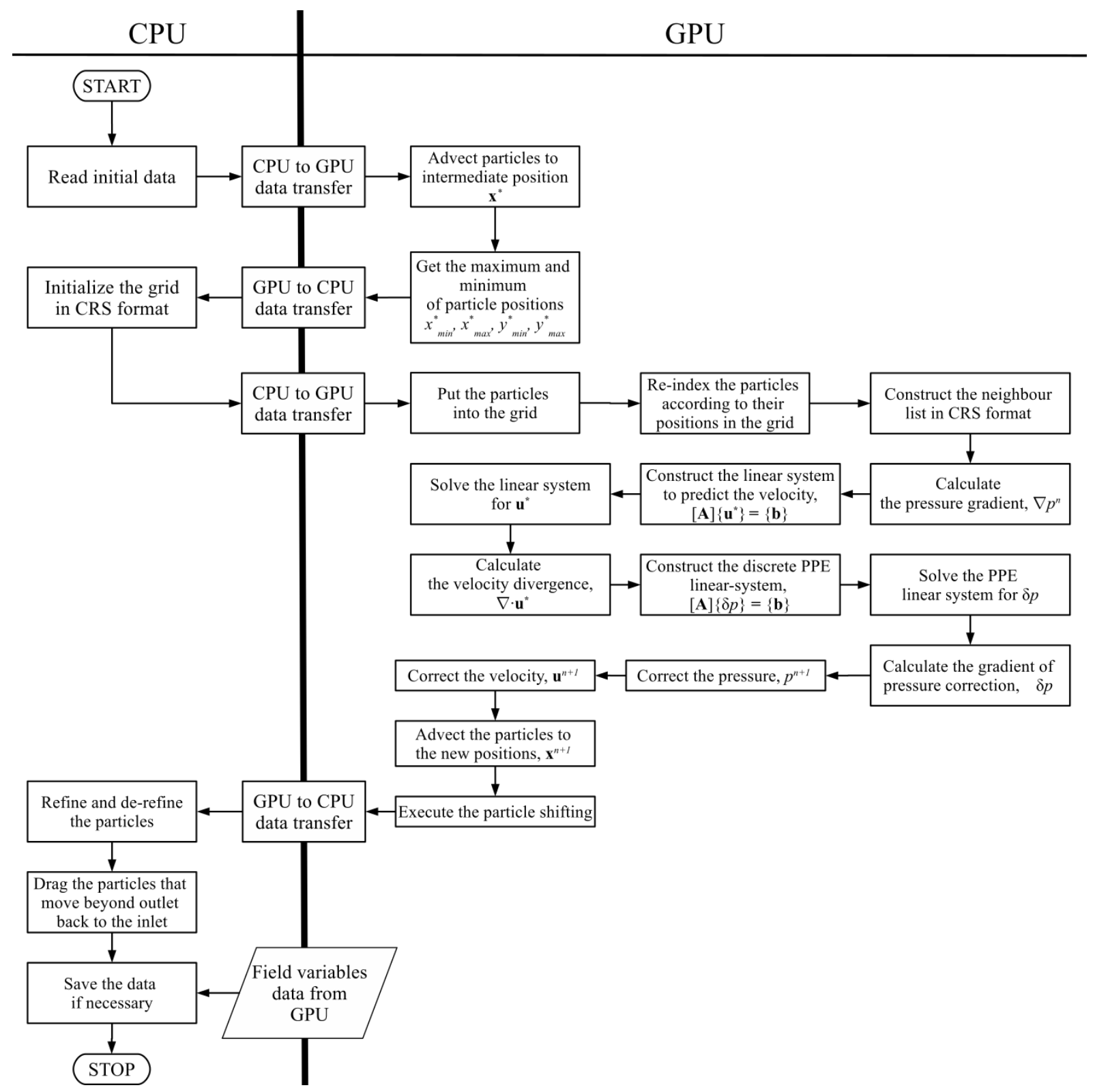

Fig. 3 Flow chart of the algorithm covering a single time step, showing the division of work and data transfers between the CPU and GPU. 

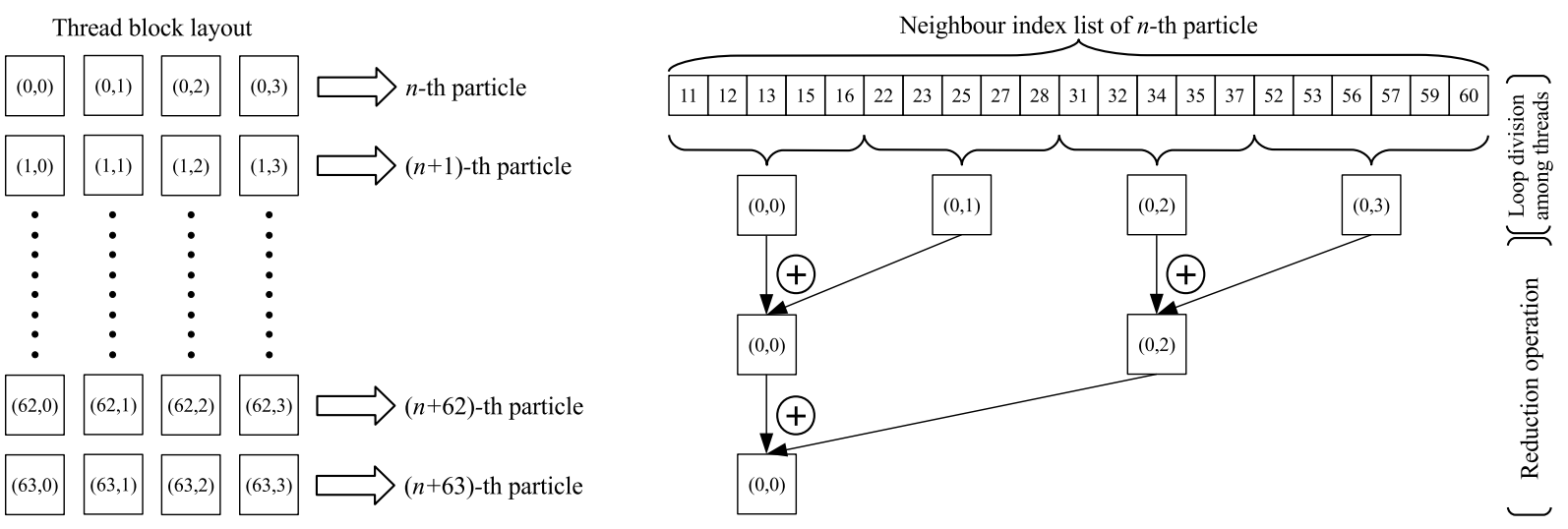

Fig. 4 Thread block layout and assignment (left), and loop division followed by reduction operation (right).

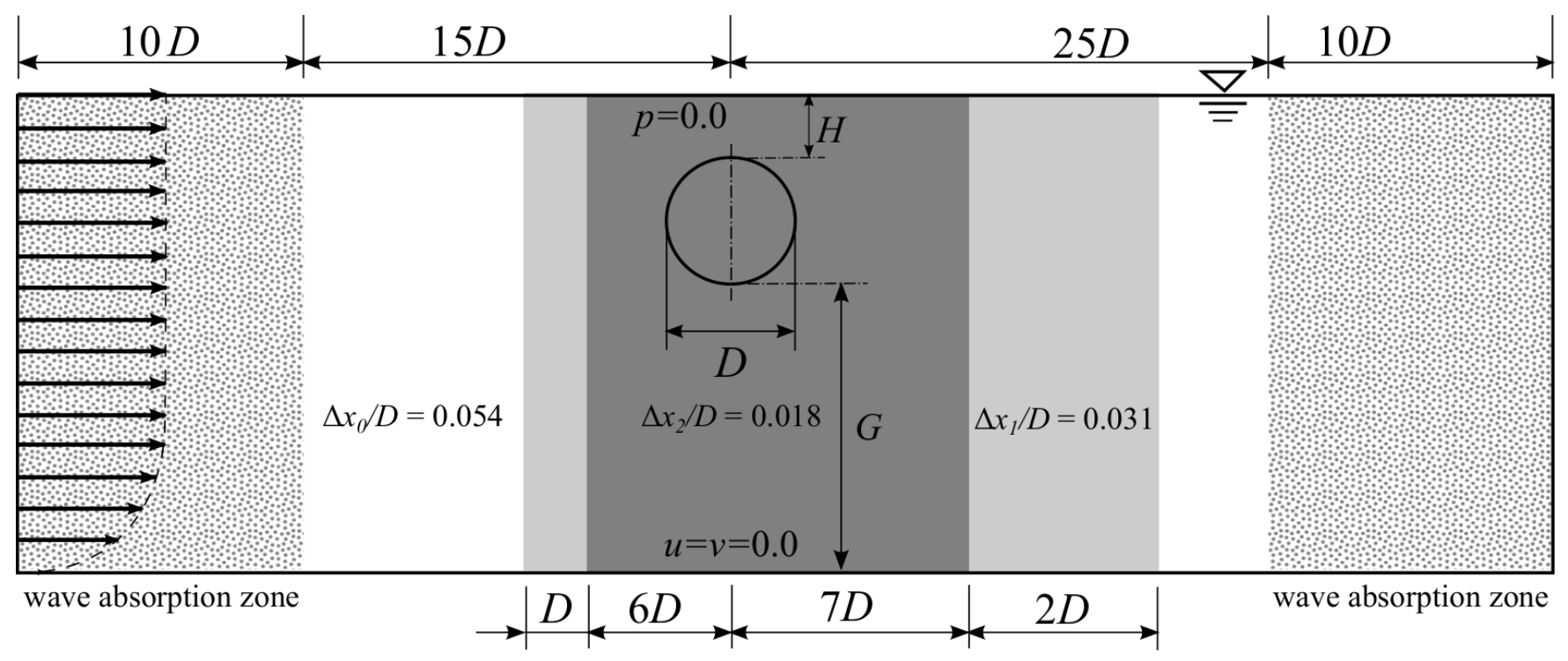

Fig. 5 Problem domain for horizontal circular cylinder with (upper) free surface and (lower) solid bed boundaries. Grey scaled rectangles represent refinement zones employed in the present study. 


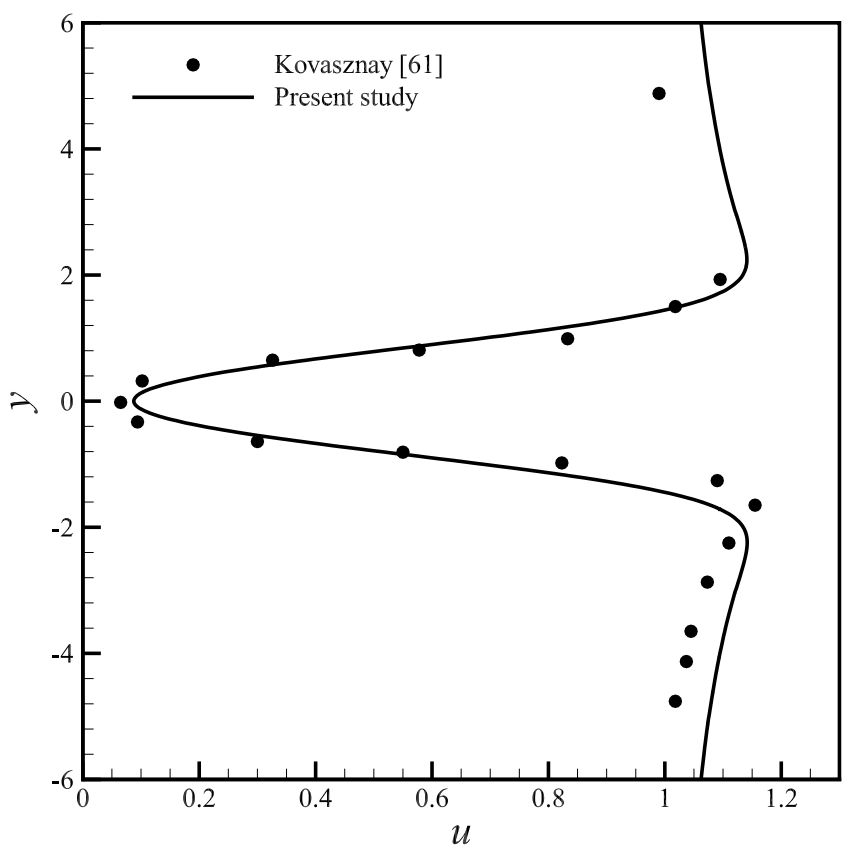

(a) $\mathrm{Re}=34$

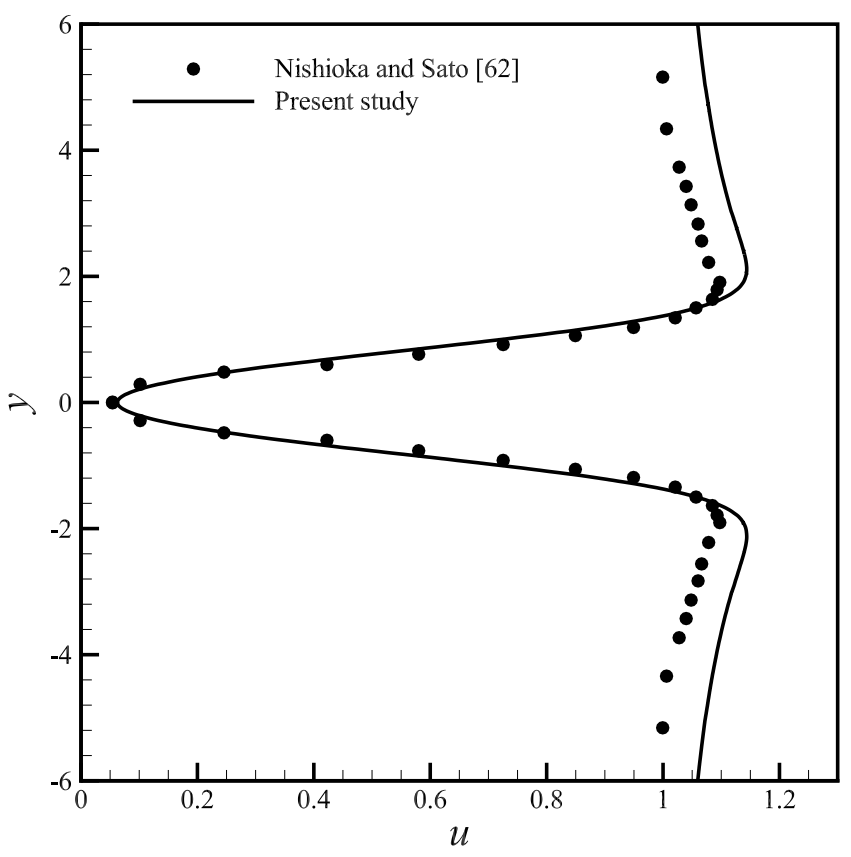

(b) $\mathrm{Re}=40$

Fig. 6 Horizontal velocity profiles at $R e=34$ (left) and 40 (right) for flow past a circular cylinder in infinite domain. 


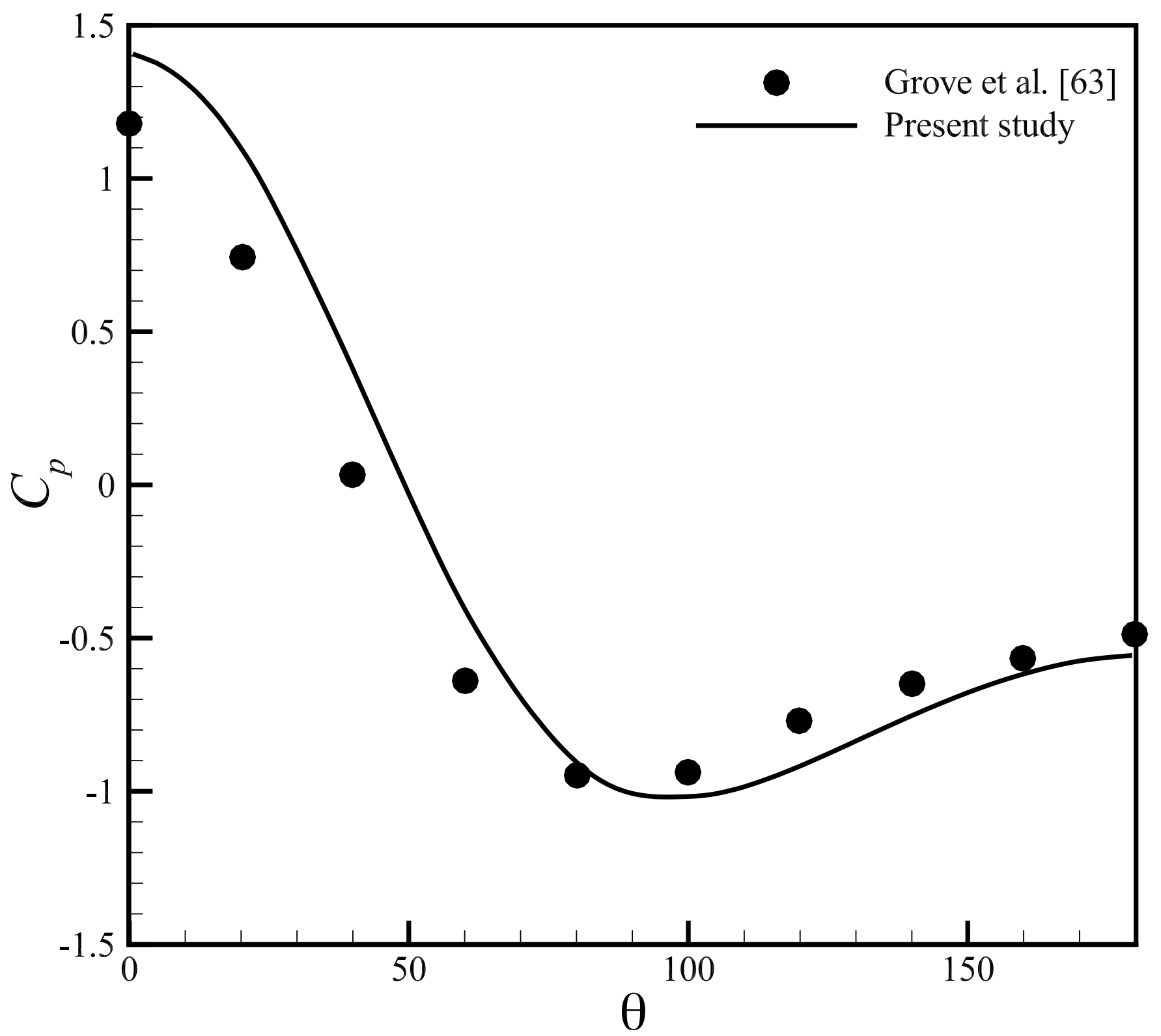

Fig. 7 Pressure coefficient along the surface of the cylinder for flow past a circular cylinder in infinite domain at $R e=40$. 


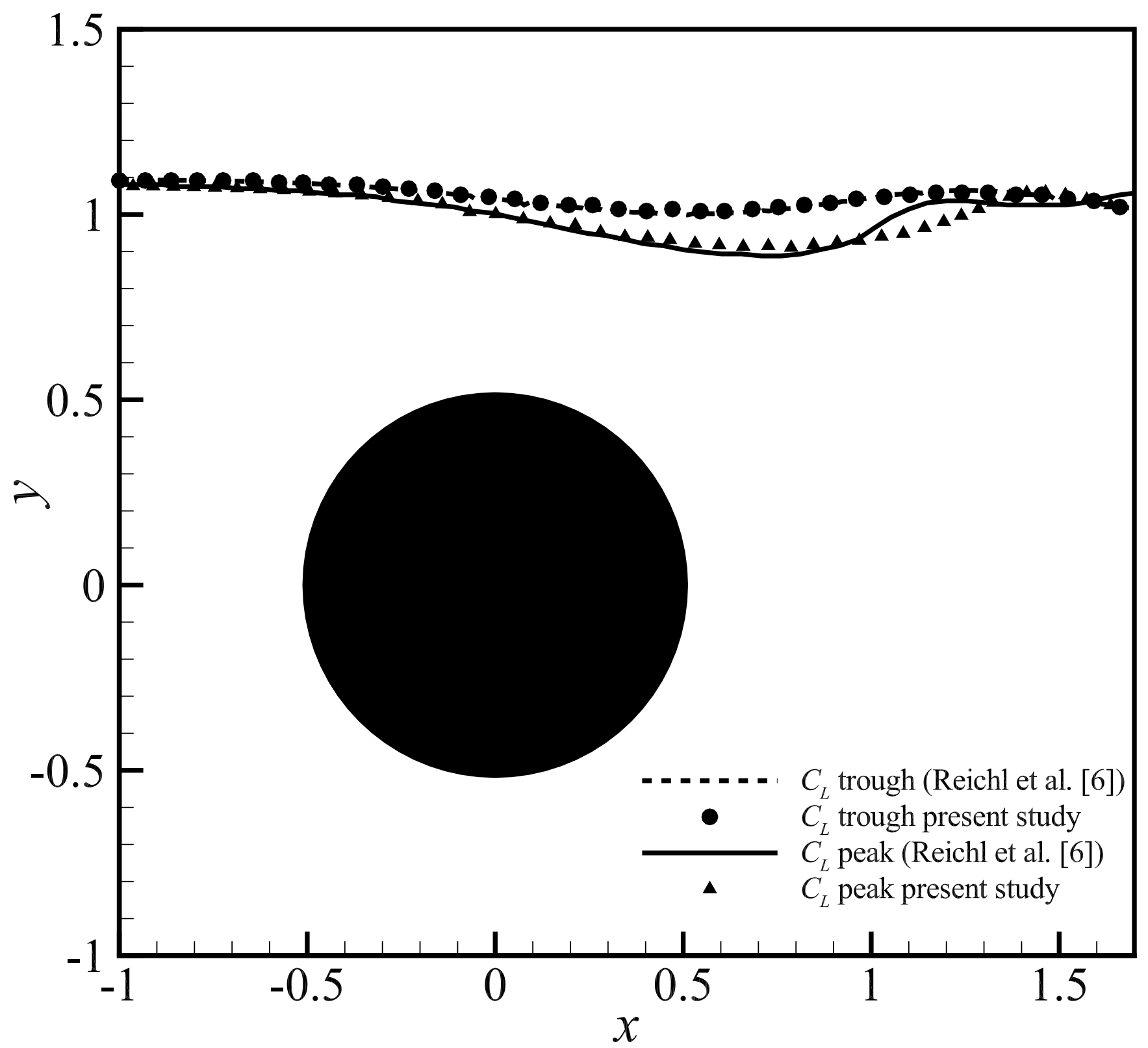

Fig. 8 Free-surface profiles near the cylinder during the peak and trough of the lift-force time-series for flow past a circular cylinder close to free surface at $\operatorname{Re}=180, \mathrm{Fr}=0.35$, and $H / D=0.55$. 

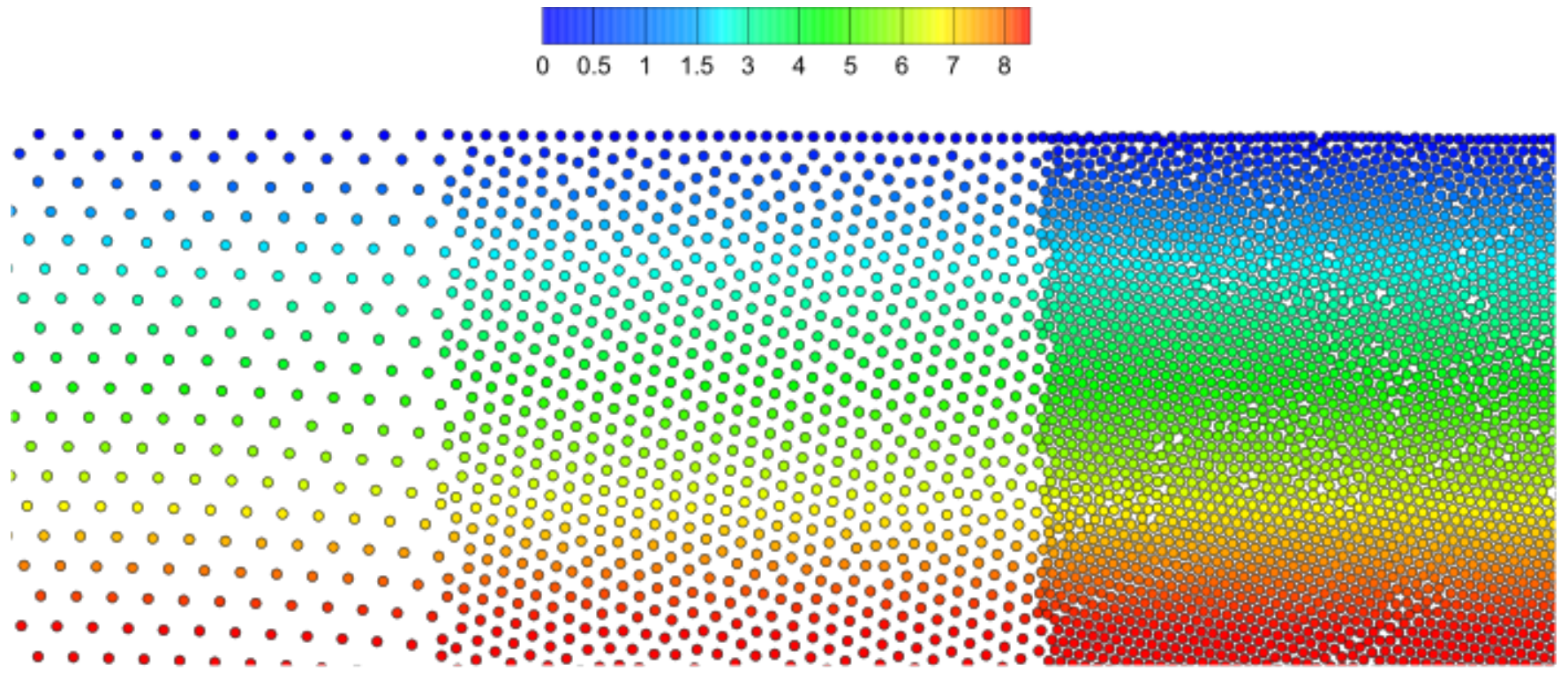

(a) Particle size transitions before the cylinder

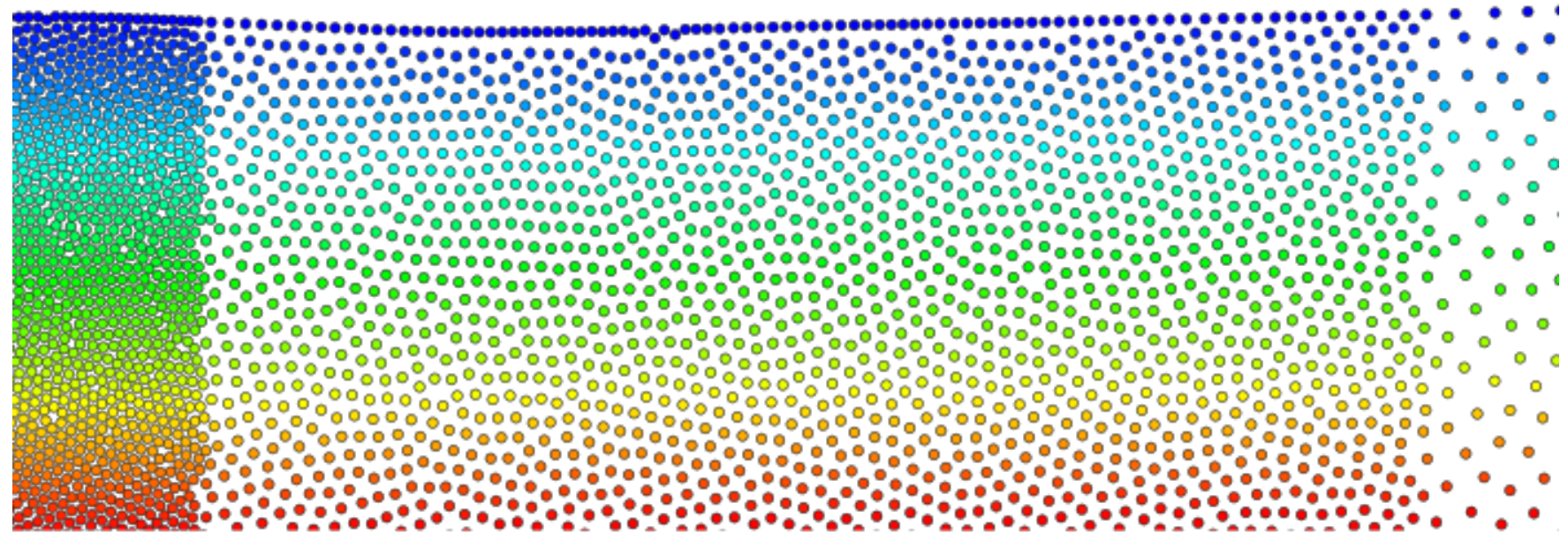

(b) Particle size transitions after the cylinder

Fig. 9 Instantaneous pressure in the vicinity of the size transition regions. 


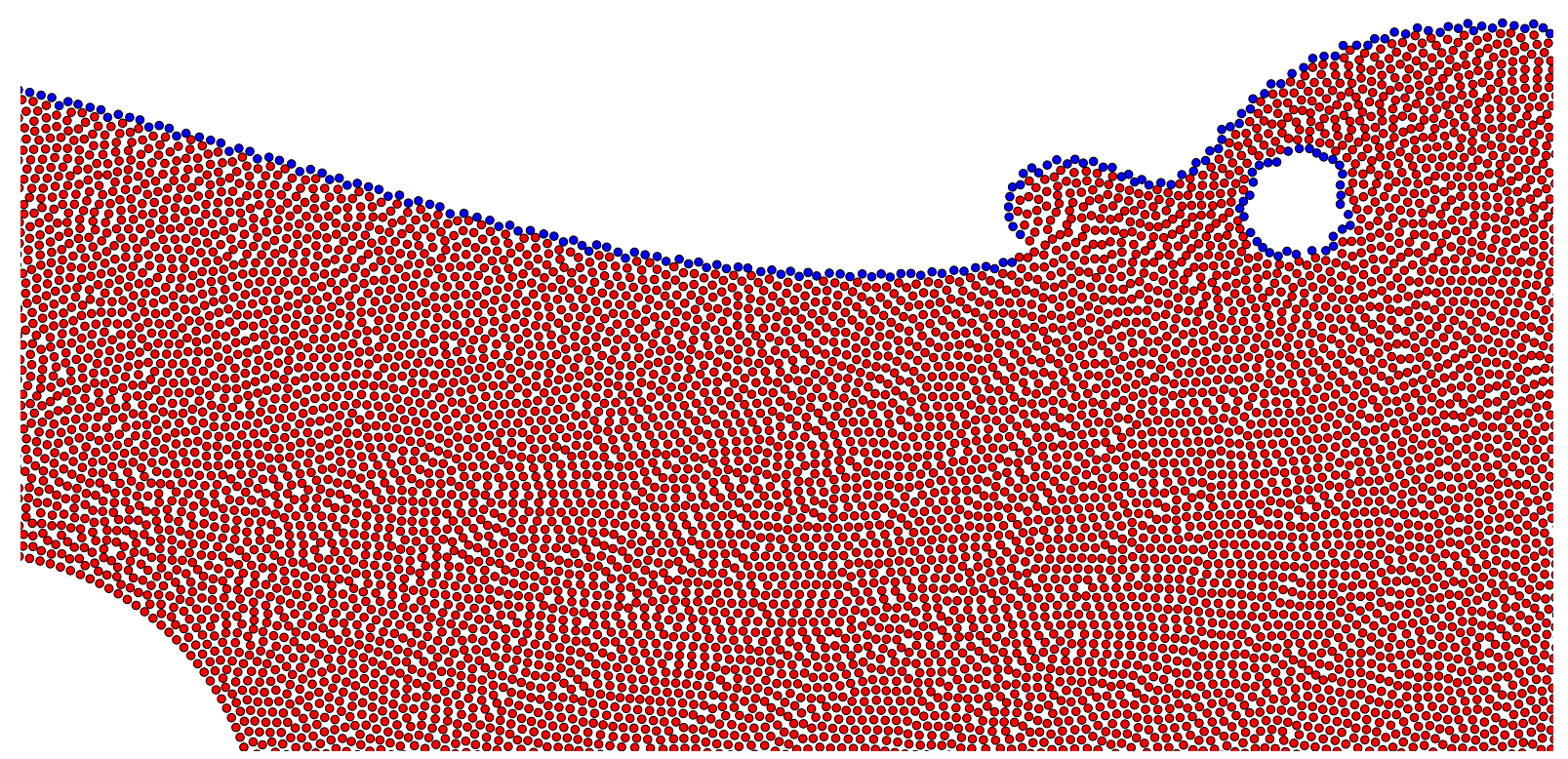

Fig. 10 Colour marked free surface $(\bullet)$ and near free surface $(\bigcirc)$ particles downstream of the cylinder in a wave breaking event. 

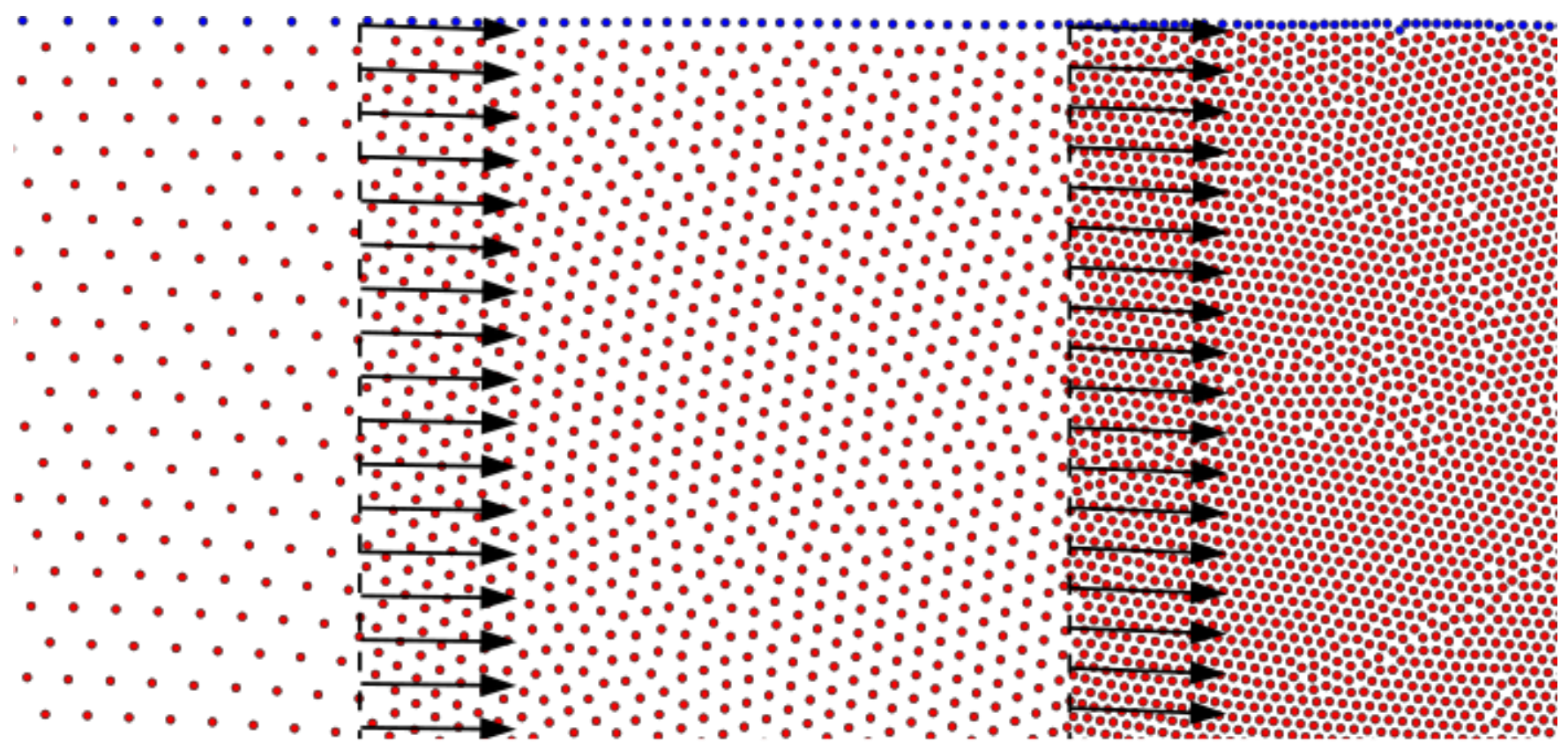

(a) Particle size transitions before the cylinder

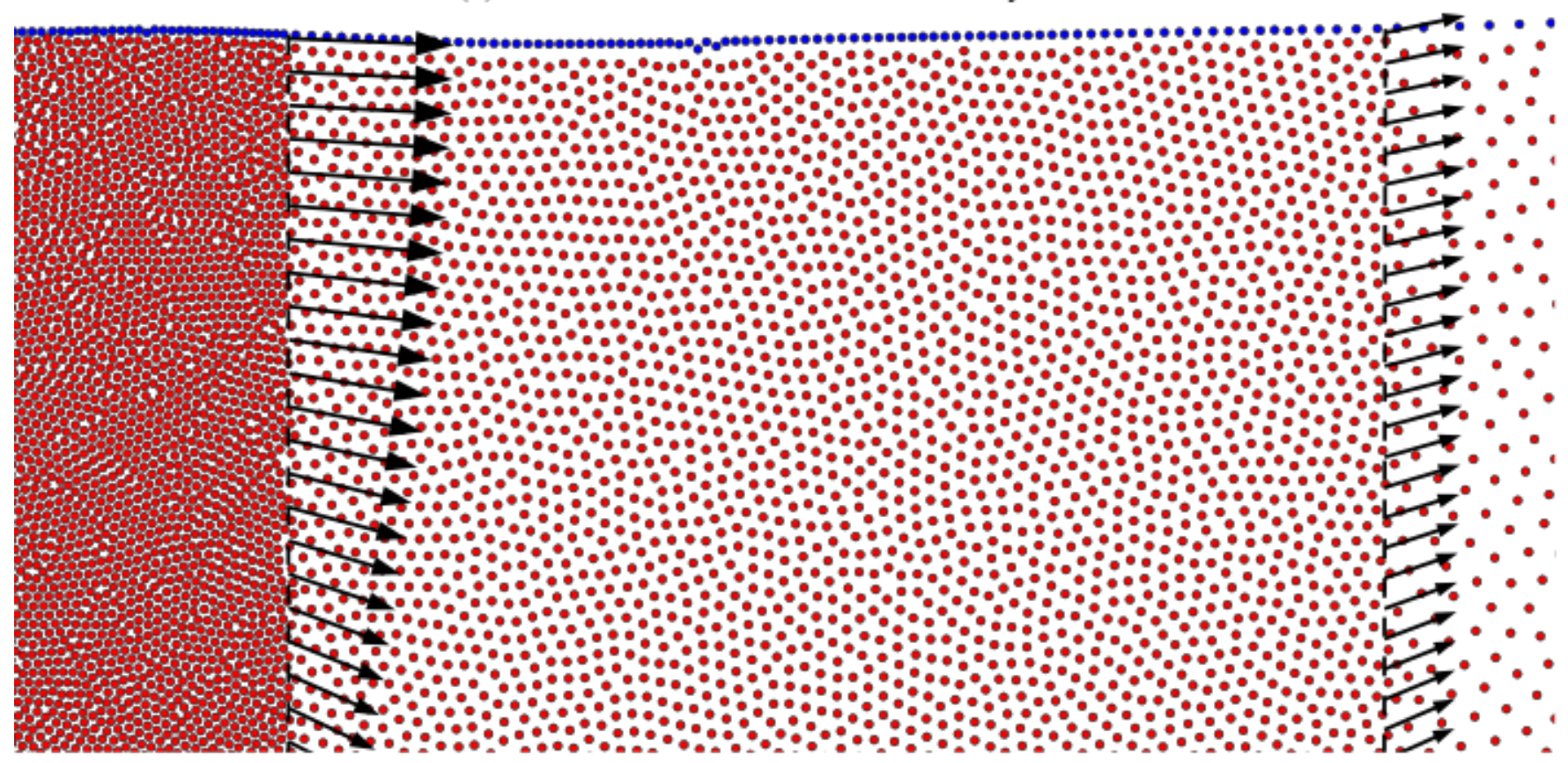

(b) Particle size transitions after the cylinder

Fig. 11 Instantaneous colour-function (Eq. (6)) distributions $(\bullet: 0$ and $\bigcirc: 1)$ in the vicinity of the size transition regions, with the velocity profiles superimposed at the particle size transition boundaries. 

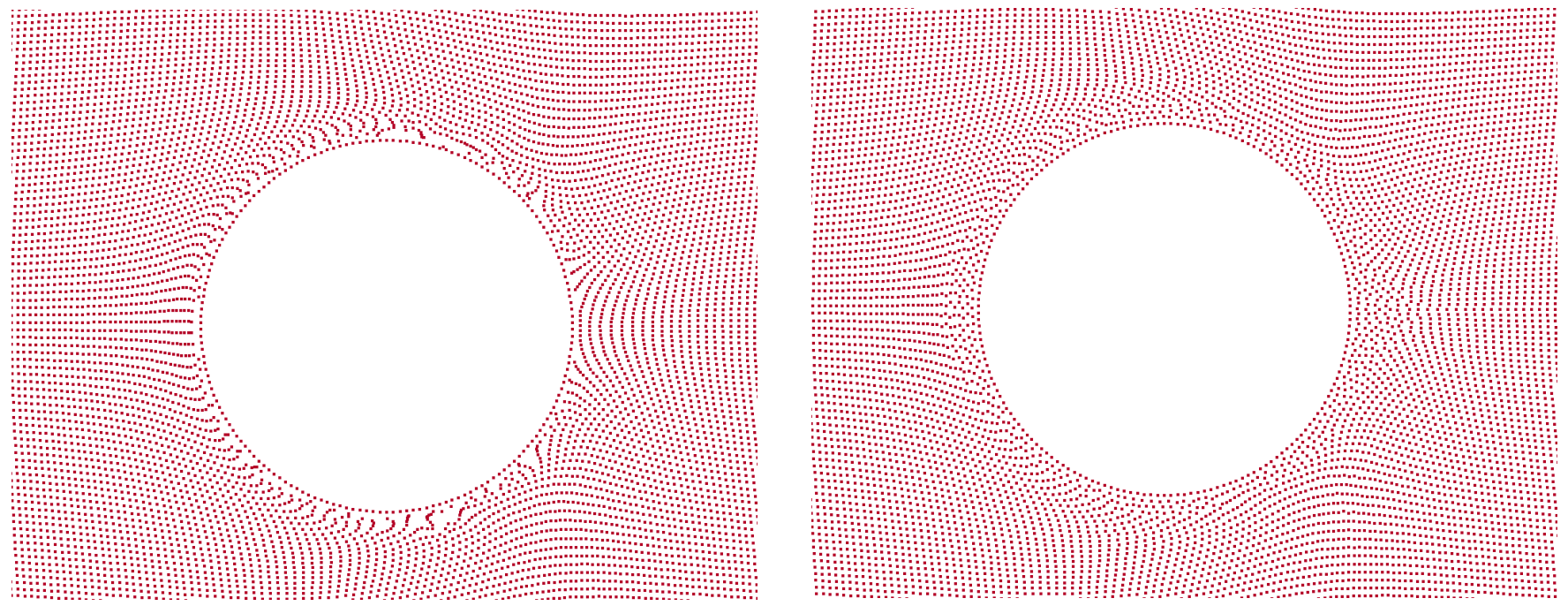

Fig. 12 Left: particle distribution around the cylinder without particle shifting, exhibiting tensile instability. Right: particle distribution around the cylinder with particle shifting.
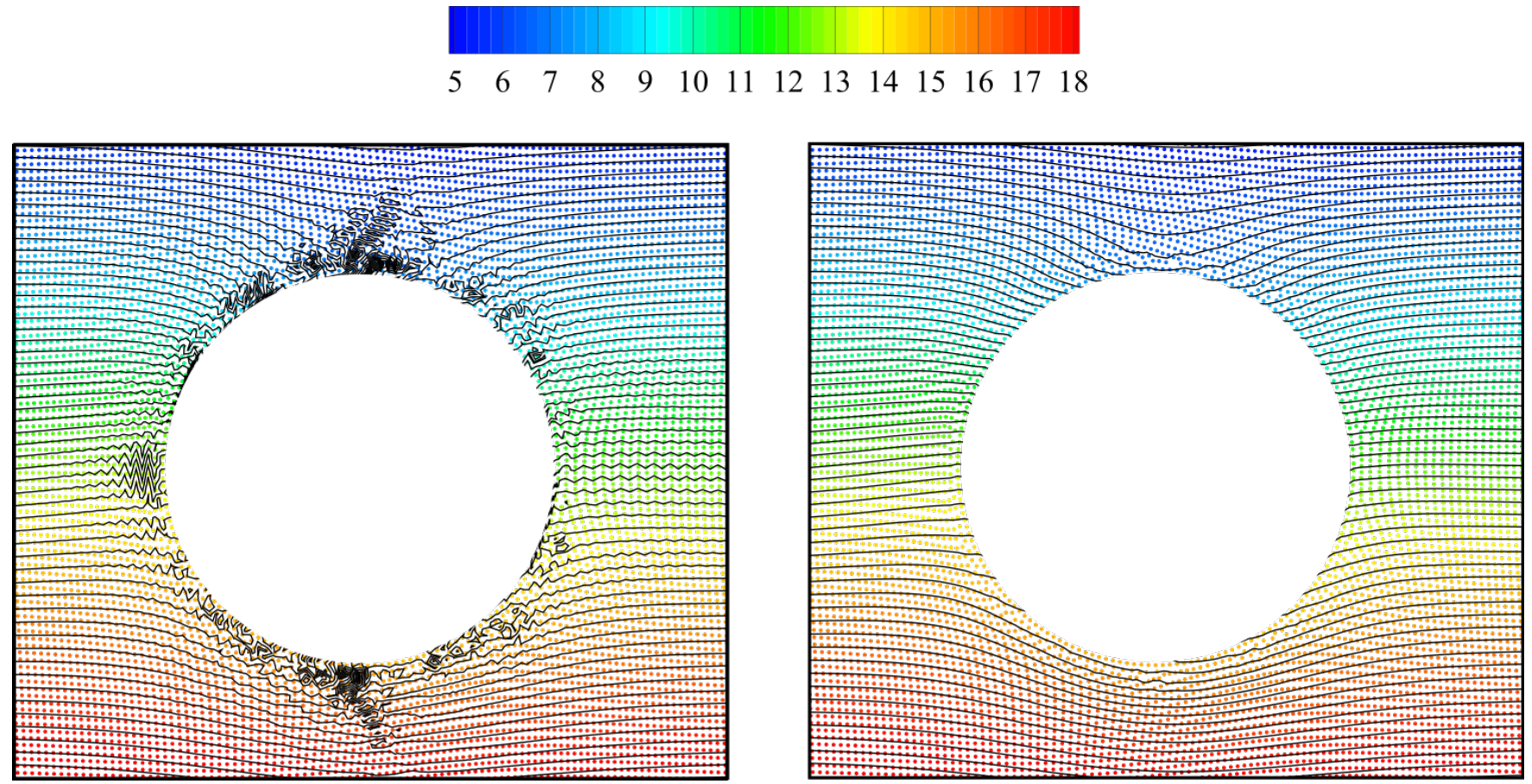

Fig. 13 Instantaneous pressure field: (Left) without Rhie and Chow interpolation, exhibiting checkerboard effect; and (Right) with Rhie and Chow interpolation. 


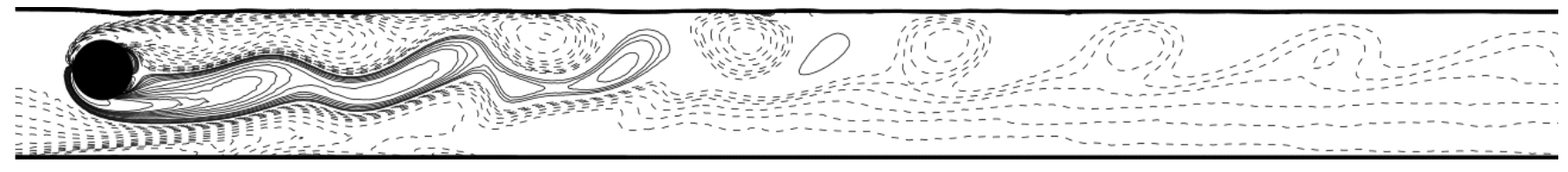

(a) $\mathrm{Fr}=0.2$

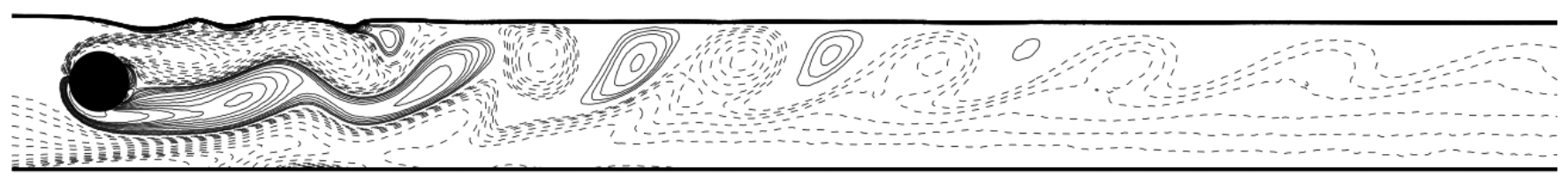

(b) $\mathrm{Fr}=0.3$

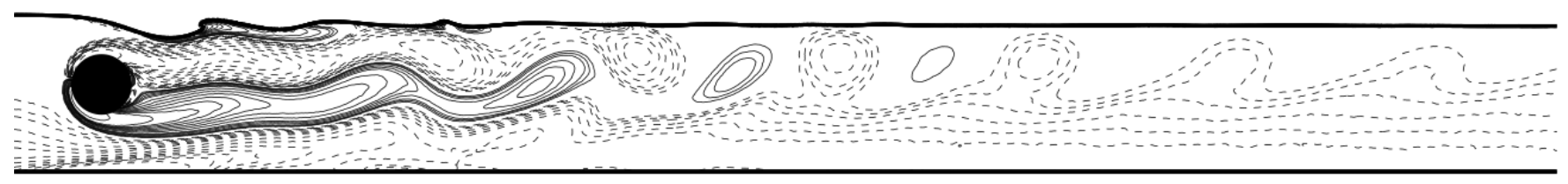

(c) $\mathrm{Fr}=0.4$

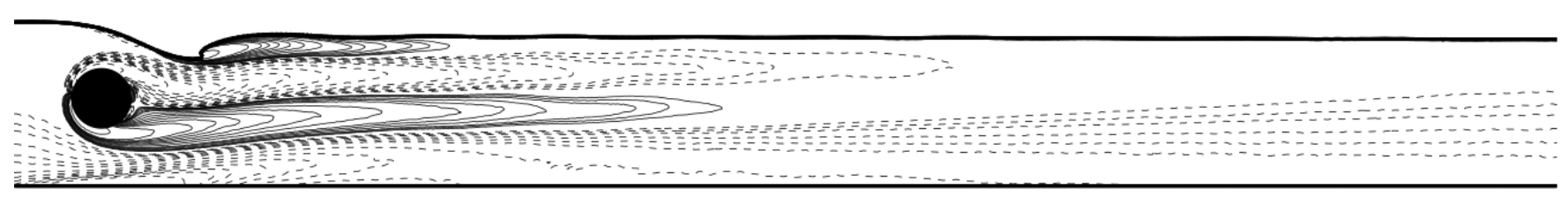

(d) $\mathrm{Fr}=0.5$

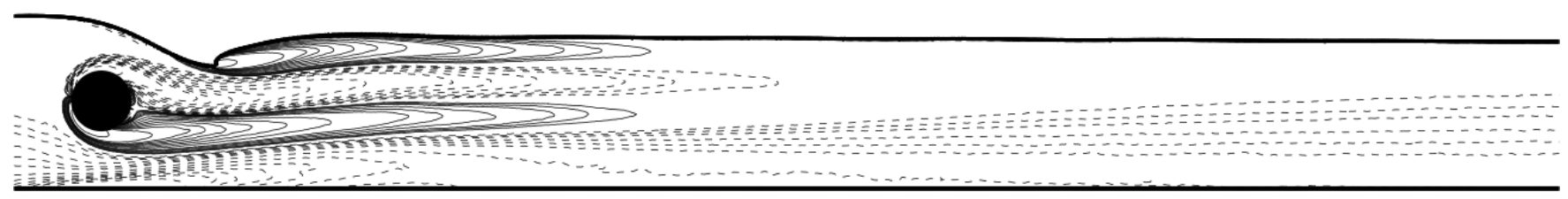

(e) $\mathrm{Fr}=0.6$

Fig. 14 Free-surface flow past a submerged horizontal cylinder: instantaneous free-surface profile and vorticity distribution for $G / D=1, H / D=0.5$, and $\mathrm{Fr}=0.2,0.3,0.4,0.5$, and 0.6 at $t=200$. Solid and dashed lines represent positive and negative vorticity, respectively. 


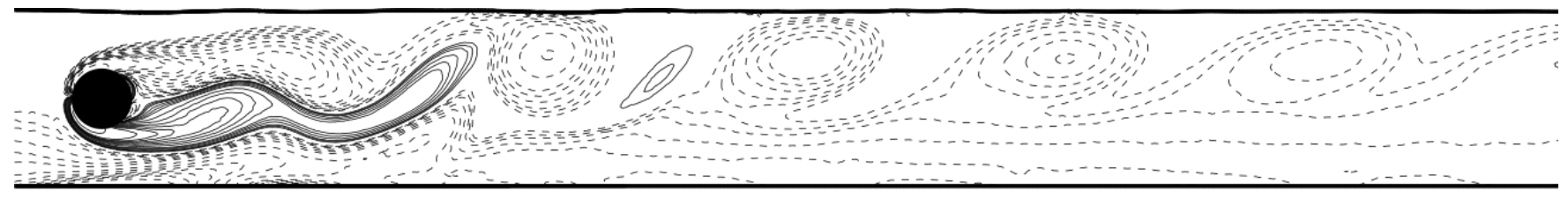

(a) $\mathrm{Fr}=0.2$

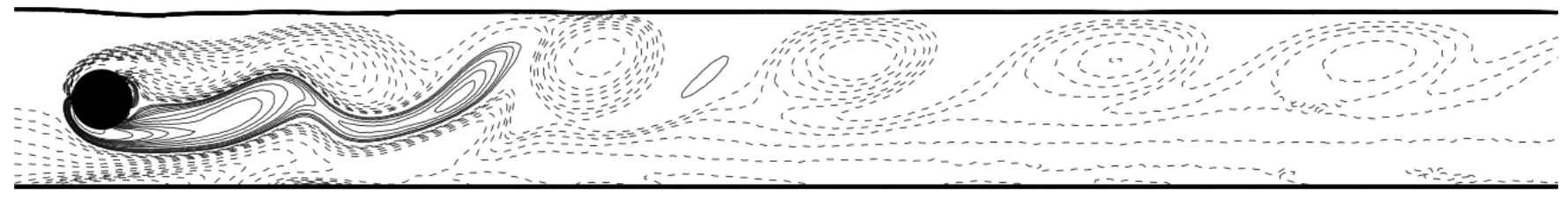

(b) $\mathrm{Fr}=0.3$

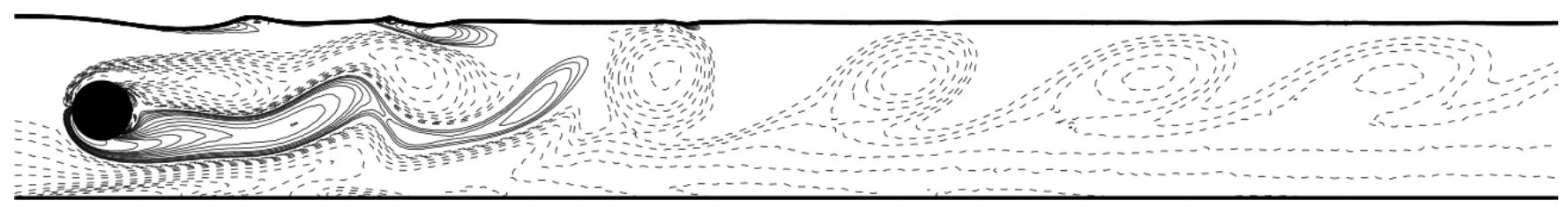

(c) $\mathrm{Fr}=0.4$

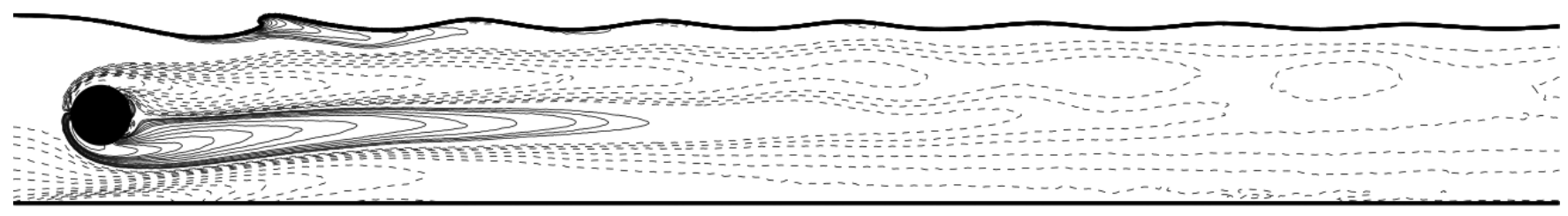

(d) $\mathrm{Fr}=0.5$

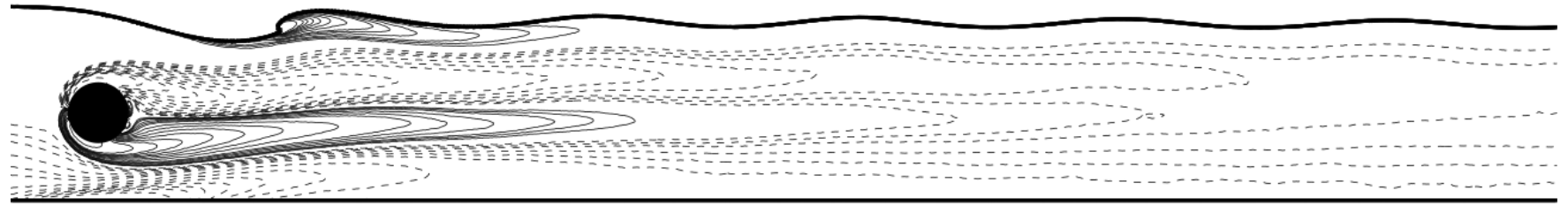

(e) $\operatorname{Fr}=0.6$

Fig. 15 Free-surface flow past a submerged horizontal cylinder: instantaneous free-surface profile and vorticity distribution for $G / D=1, H / D=1$, and $\mathrm{Fr}=0.2,0.3,0.4,0.5$, and 0.6 at $t=200$. Solid and dashed lines represent positive and negative vorticity, respectively. 


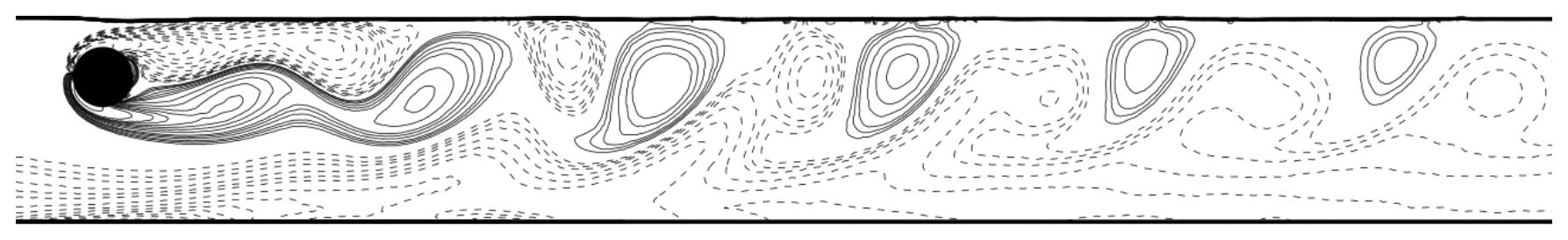

(a) $\mathrm{Fr}=0.2$

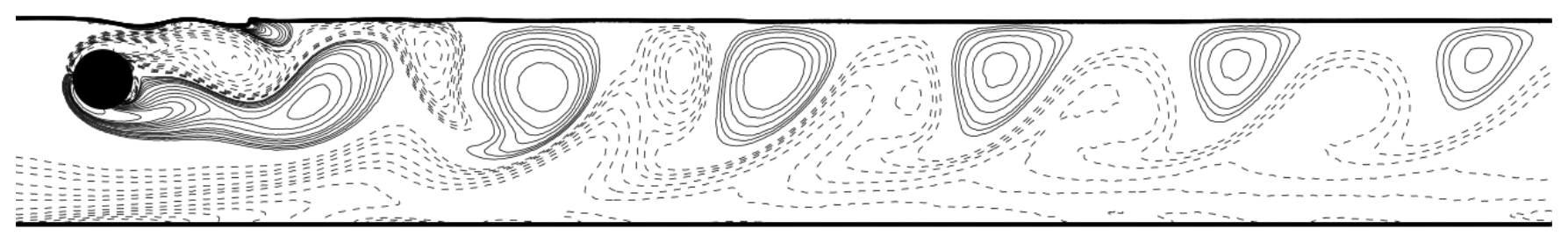

(b) $\mathrm{Fr}=0.3$

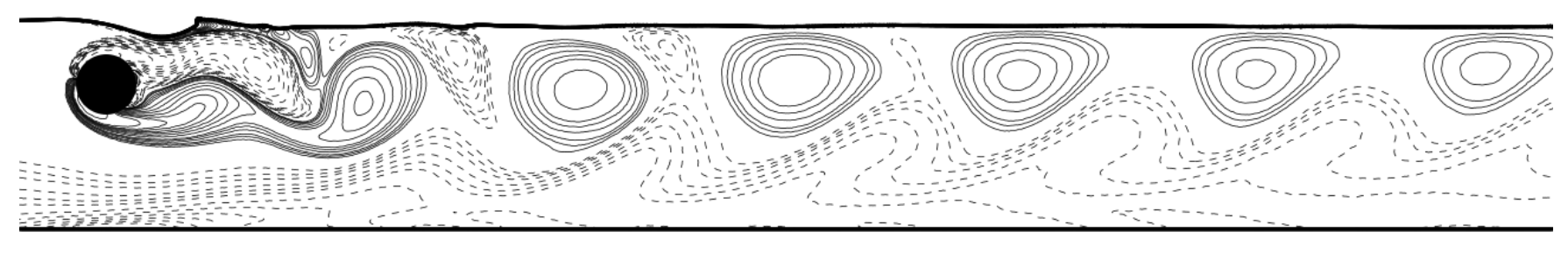

(c) $\mathrm{Fr}=0.4$

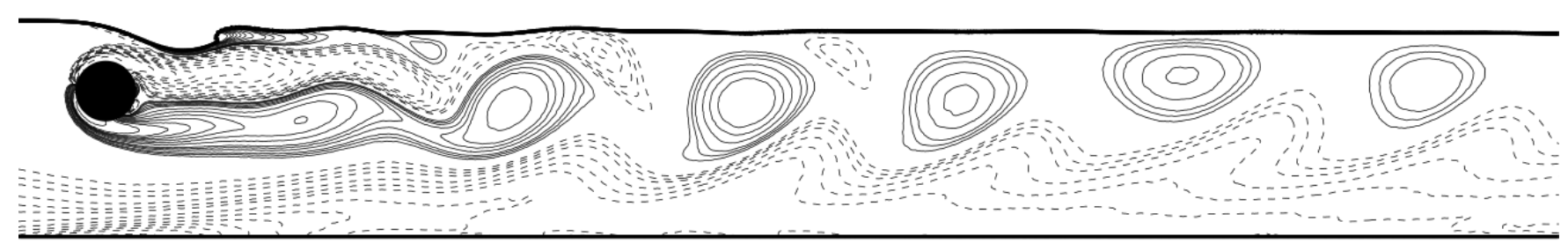

(d) $\mathrm{Fr}=0.5$

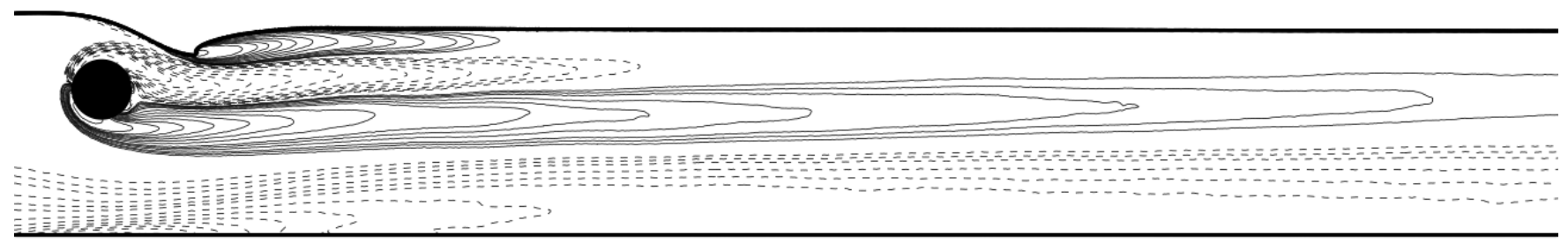

(e) $\mathrm{Fr}=0.6$

Fig. 16 Free-surface flow past a submerged horizontal cylinder: instantaneous free-surface profile and vorticity distribution for $G / D=2, H / D=0.5$, and $\mathrm{Fr}=0.2,0.3,0.4,0.5$, and 0.6 at $t=200$. Solid and dashed lines represent positive and negative vorticity, respectively. 


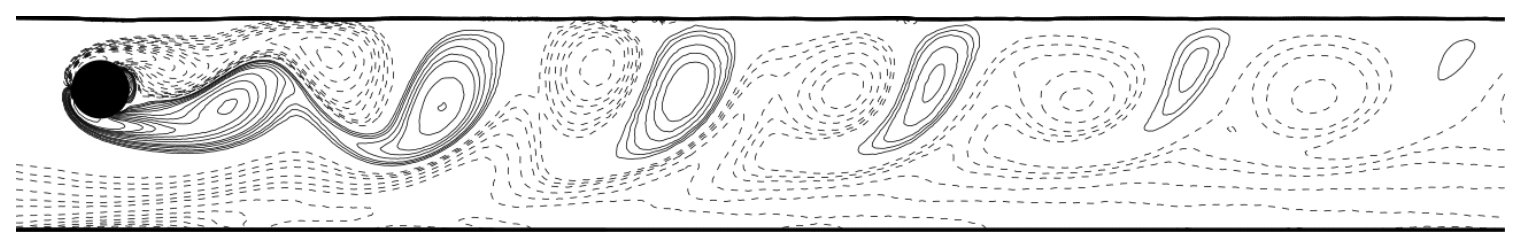

(a) $\mathrm{Fr}=0.2$

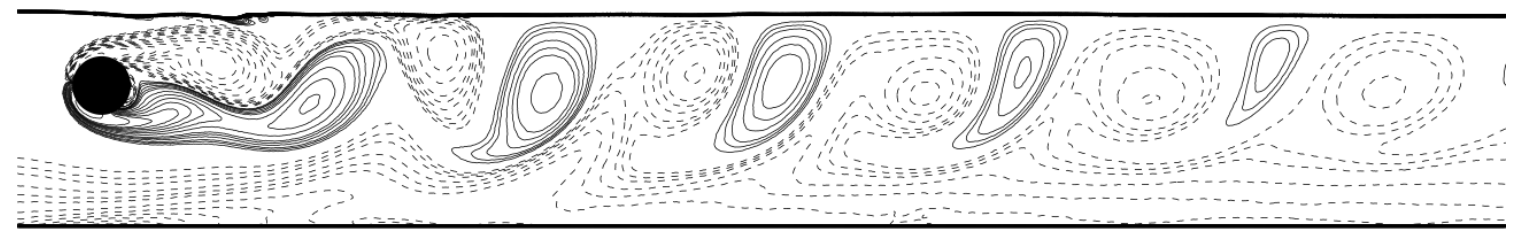

(b) $\mathrm{Fr}=0.3$

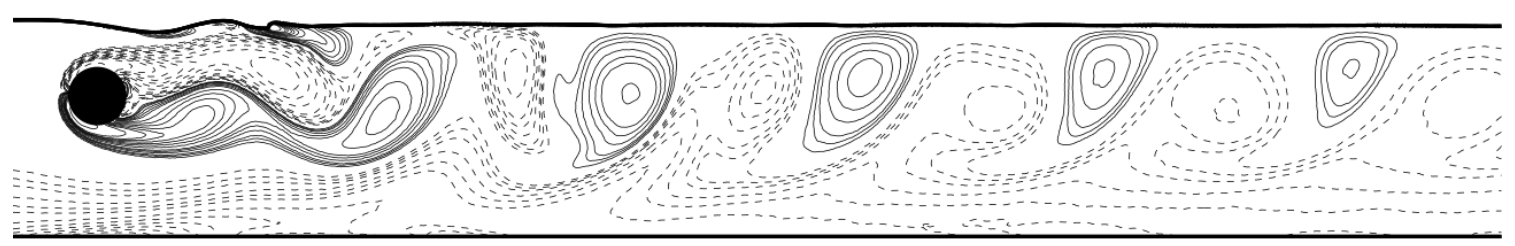

(c) $\mathrm{Fr}=0.4$

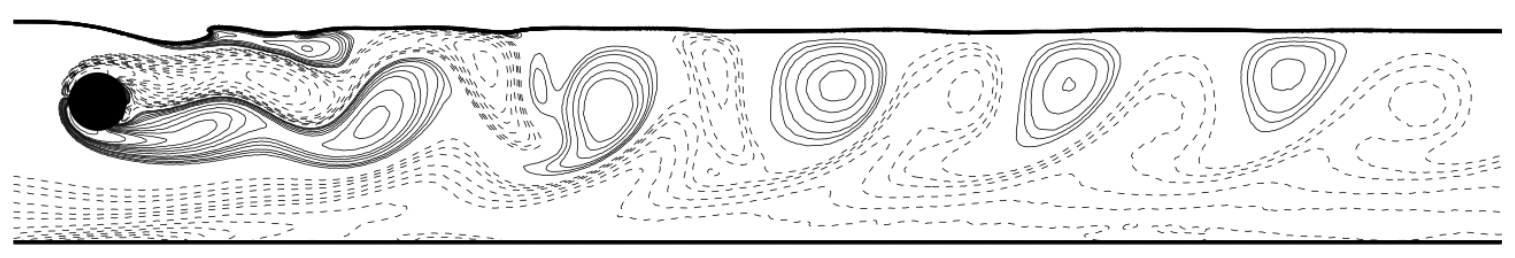

(d) $\mathrm{Fr}=0.5$

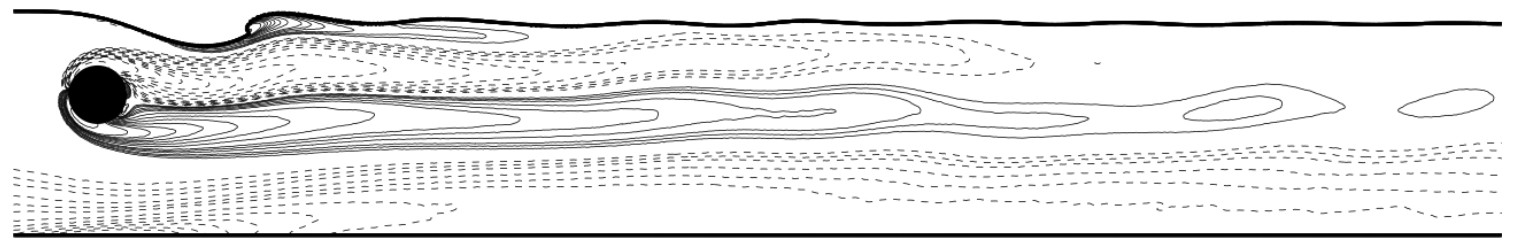

(e) $\mathrm{Fr}=0.6$

Fig. 17 Free-surface flow past a submerged horizontal cylinder: instantaneous free-surface profile and vorticity distribution for $G / D=2, H / D=0.75$, and $F r=0.2,0.3,0.4,0.5$, and 0.6 at $t=200$. Solid and dashed lines represent positive and negative vorticity, respectively. 


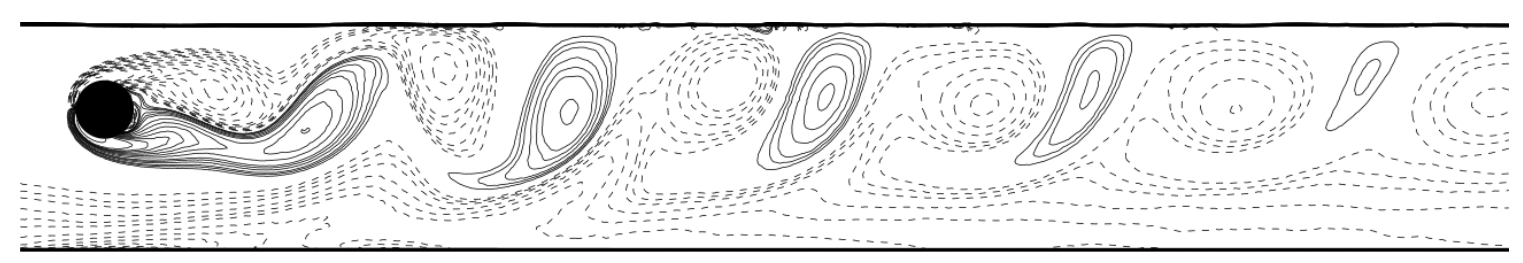

(a) $\mathrm{Fr}=0.2$

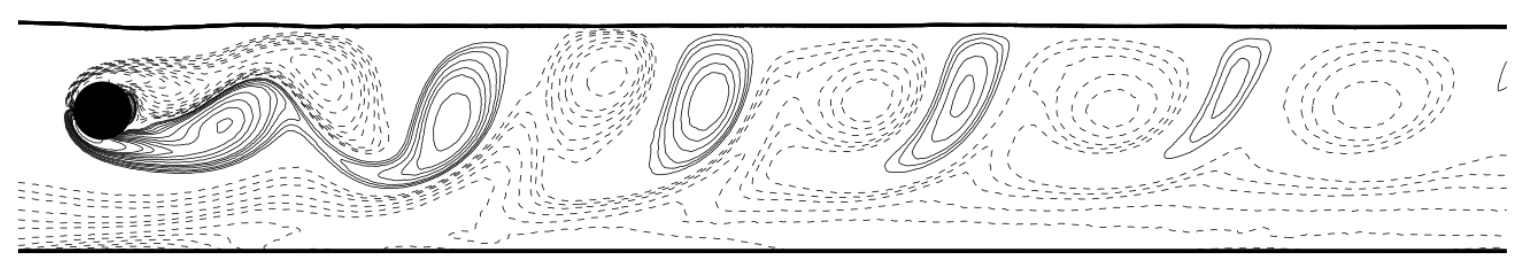

(b) $\mathrm{Fr}=0.3$

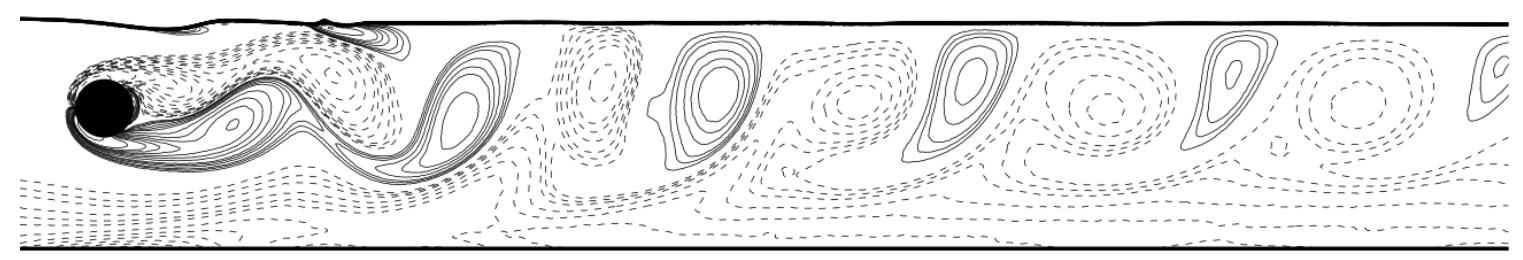

(c) $\mathrm{Fr}=0.4$

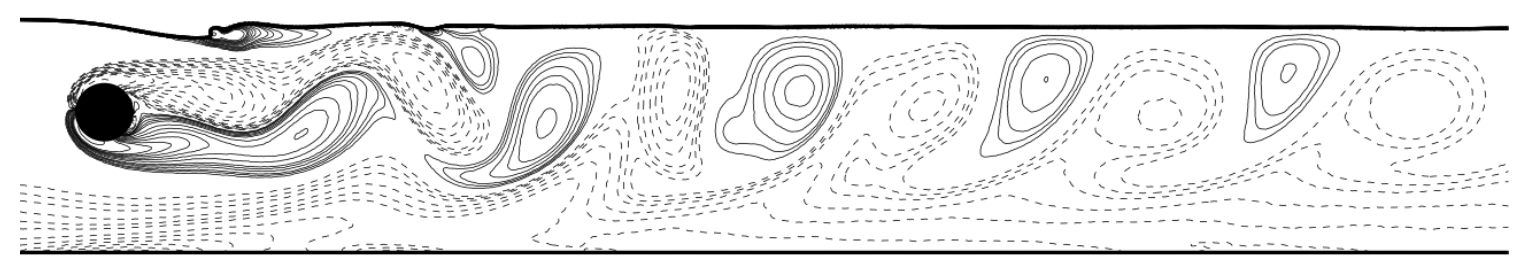

(d) $\mathrm{Fr}=0.5$

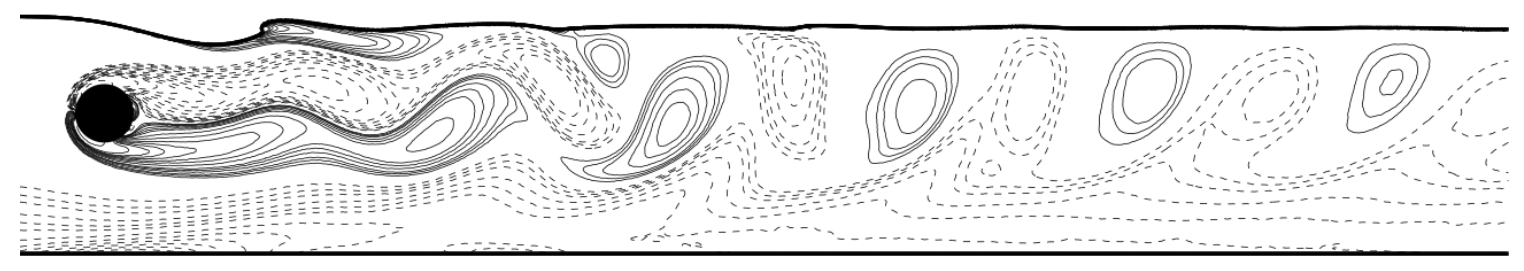

(e) $\mathrm{Fr}=0.6$

Fig. 18 Free-surface flow past a submerged horizontal cylinder: instantaneous free-surface profile and vorticity distribution for $G / D=2, H / D=1$, and $\mathrm{Fr}=0.2,0.3,0.4,0.5$, and 0.6 at $t=200$. Solid and dashed lines represent positive and negative vorticity, respectively. 


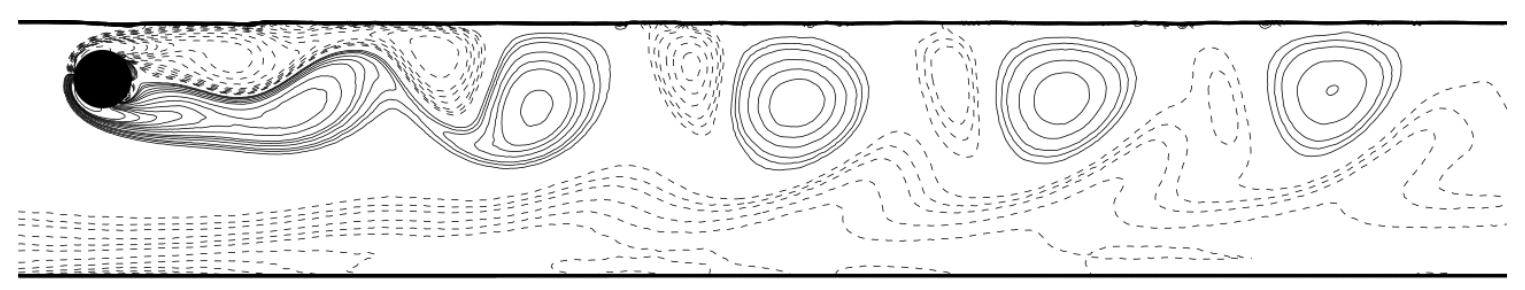

(a) $\operatorname{Fr}=0.2$

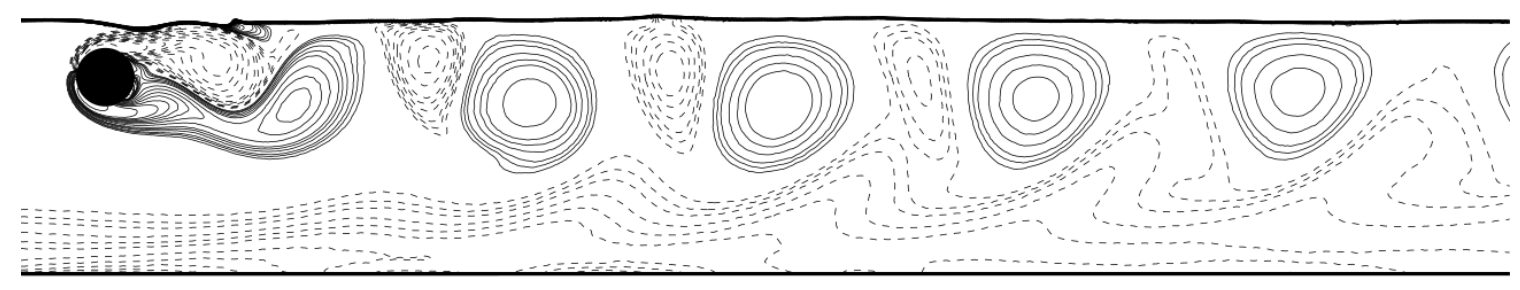

(b) $\mathrm{Fr}=0.3$

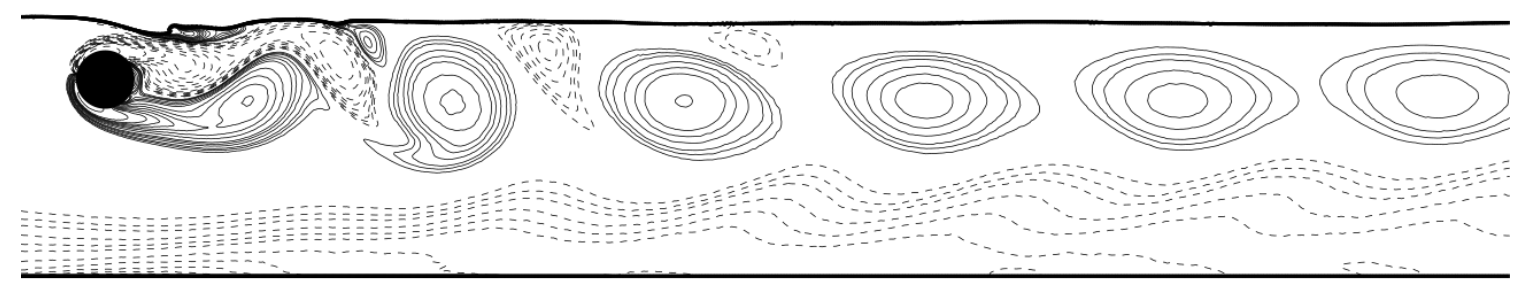

(c) $\mathrm{Fr}=0.4$

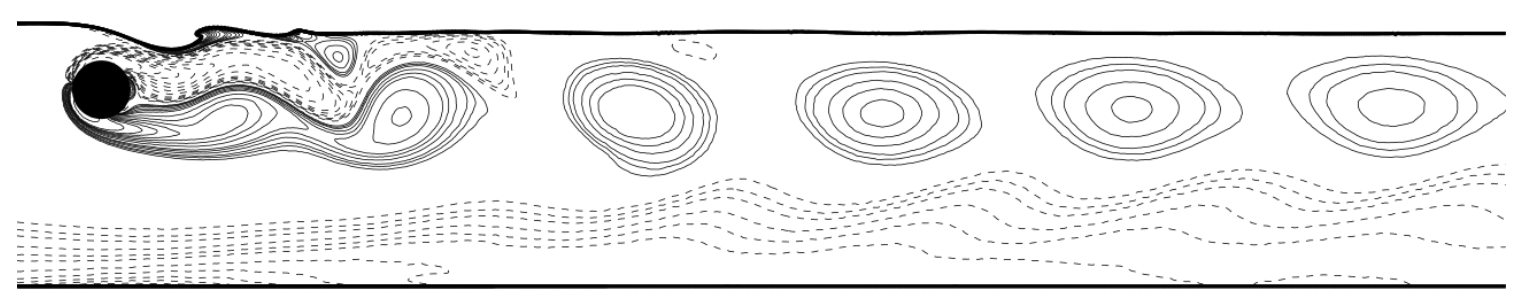

(d) $\mathrm{Fr}=0.5$

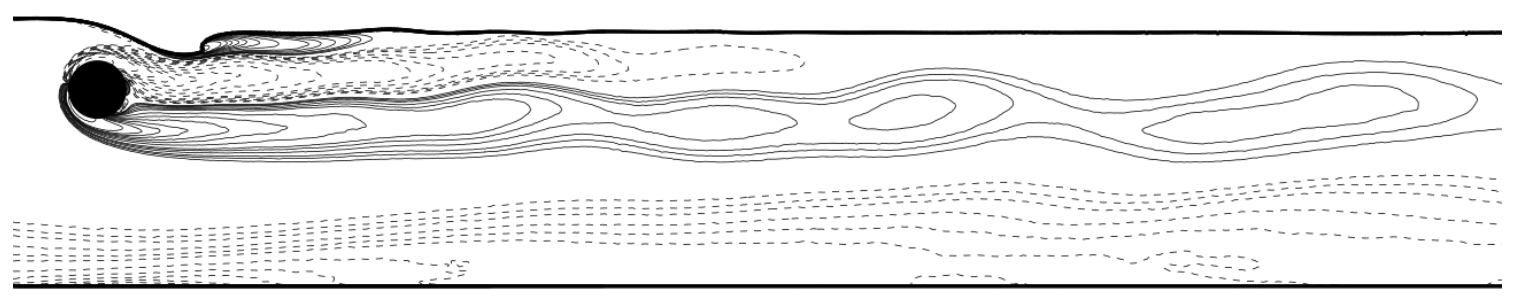

(e) $\mathrm{Fr}=0.6$

Fig. 19 Free-surface flow past a submerged horizontal cylinder: instantaneous free-surface profile and vorticity distribution for $G / D=3, H / D=0.5$, and $F r=0.2,0.3,0.4,0.5$, and 0.6 at $t=200$. Solid and dashed lines represent positive and negative vorticity, respectively. 


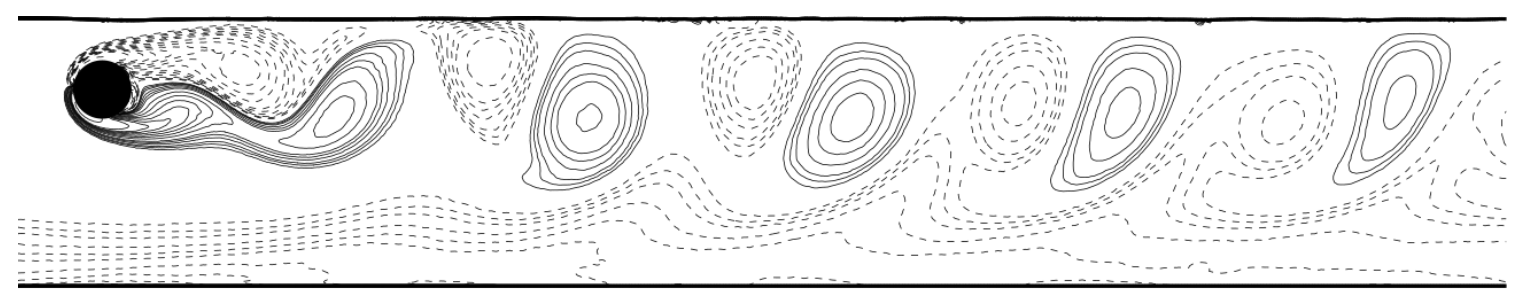

(a) $\mathrm{Fr}=0.2$

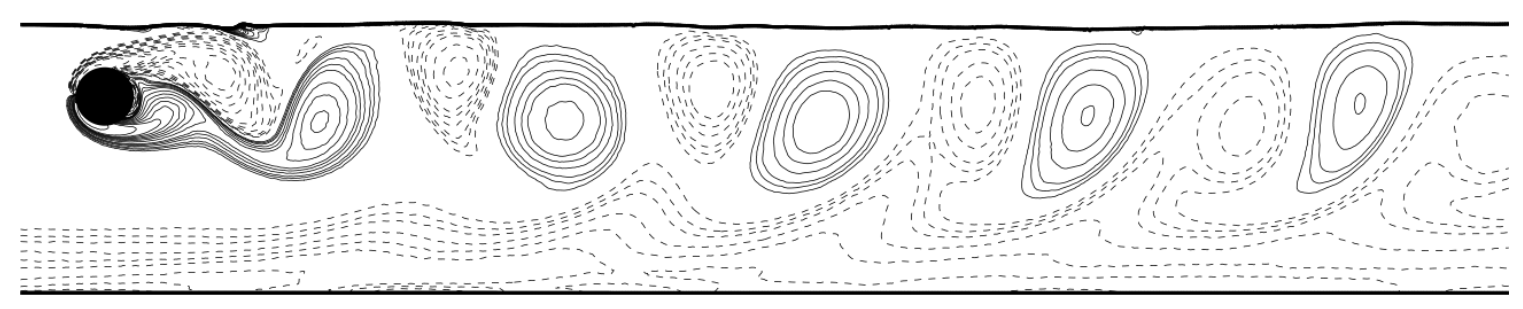

(b) $\mathrm{Fr}=0.3$

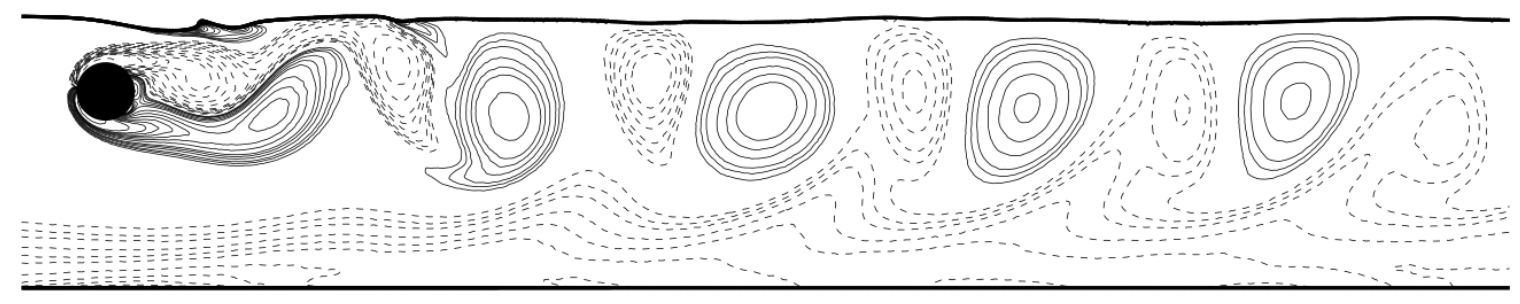

(c) $\mathrm{Fr}=0.4$

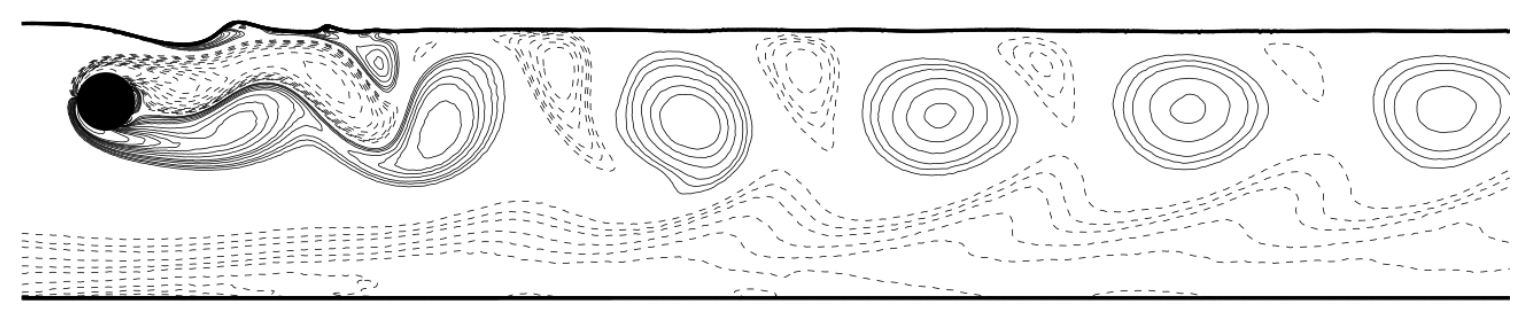

(d) $\mathrm{Fr}=0.5$

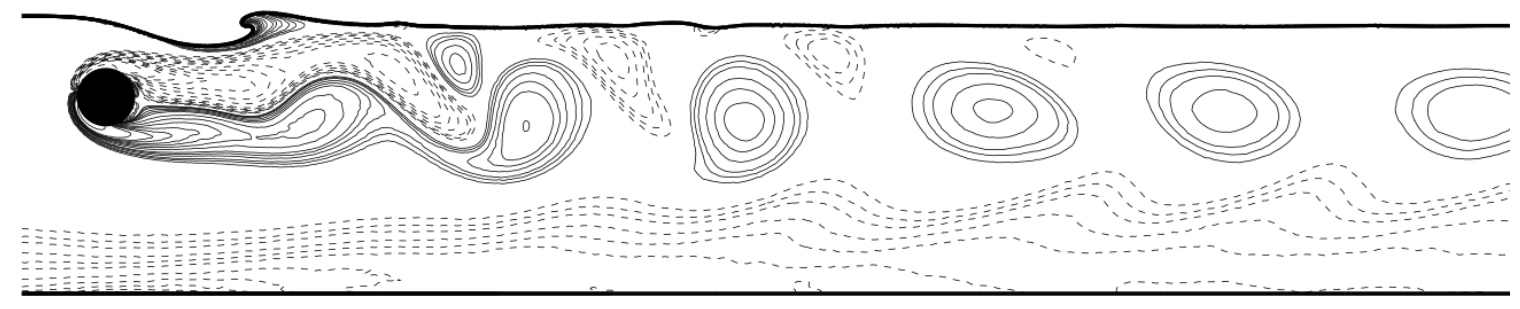

(e) $\mathrm{Fr}=0.6$

Fig. 20 Free-surface flow past a submerged horizontal cylinder: instantaneous free-surface profile and vorticity distribution for $G / D=3, H / D=0.75$, and $\mathrm{Fr}=0.2,0.3,0.4,0.5$, and 0.6 at $t=200$. Solid and dashed lines represent positive and negative vorticity, respectively. 


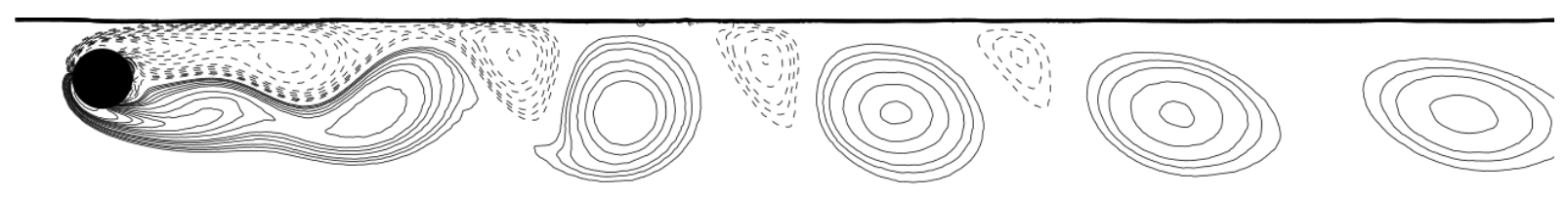

(a) $\mathrm{Fr}=0.2$

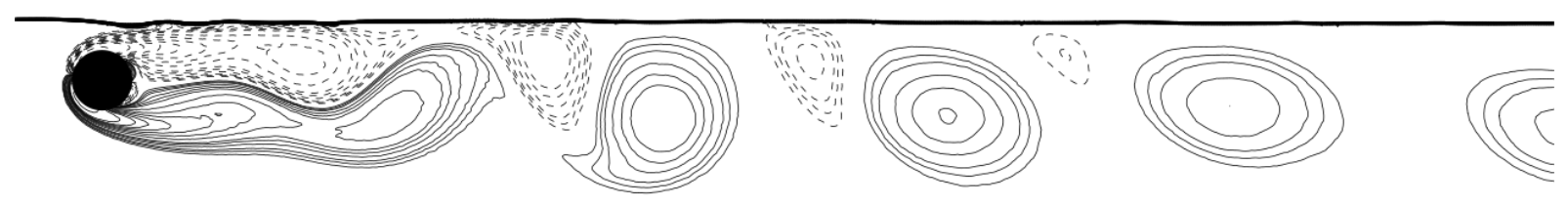

(b) $\mathrm{Fr}=0.3$

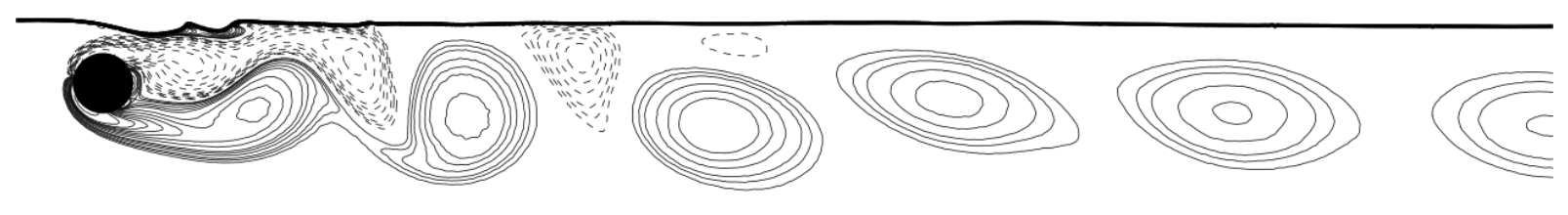

(c) $\mathrm{Fr}=0.4$

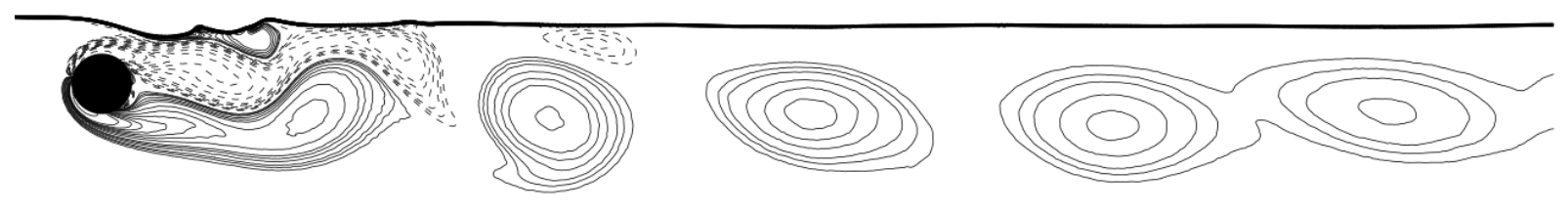

(d) $\mathrm{Fr}=0.5$

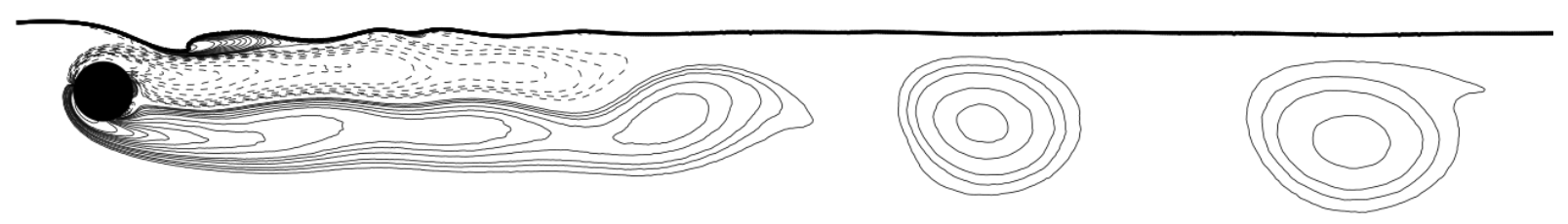

(e) $\mathrm{Fr}=0.6$

Fig. 21 Free-surface flow past a submerged horizontal cylinder: instantaneous free-surface profile and vorticity distribution for $G / D=5, H / D=0.5$, and $\mathrm{Fr}=0.2,0.3,0.4,0.5$, and 0.6 at $t=200$. Solid and dashed lines represent positive and negative vorticity, respectively. 


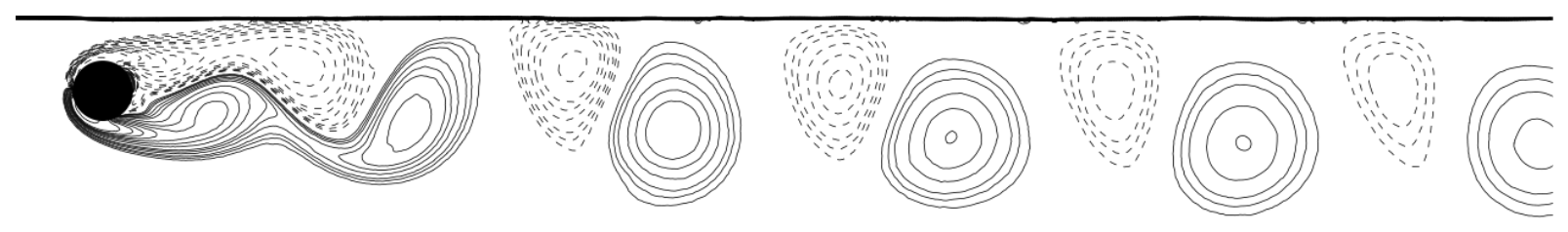

(a) $\mathrm{Fr}=0.2$

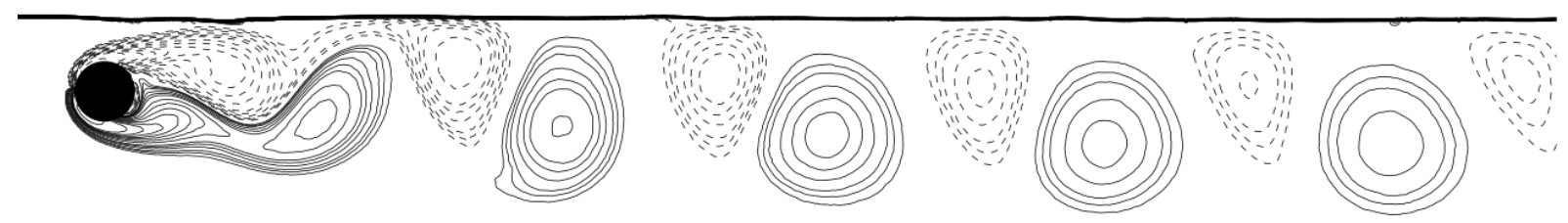

(b) $\mathrm{Fr}=0.3$

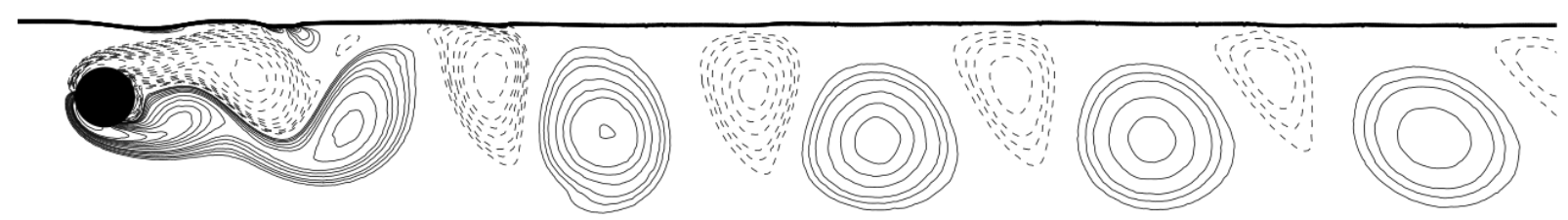

(c) $\mathrm{Fr}=0.4$

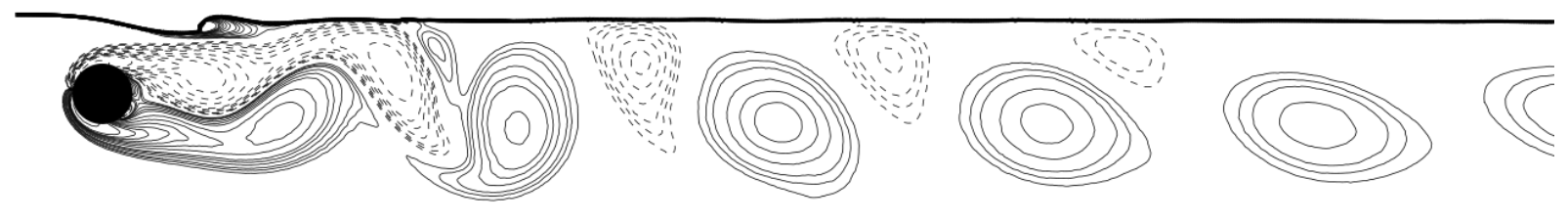

(d) $\mathrm{Fr}=0.5$

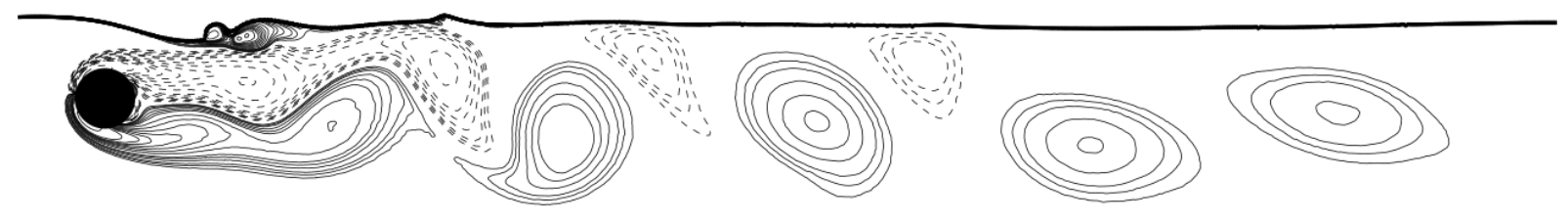

(e) $\mathrm{Fr}=0.6$

Fig. 22 Free-surface flow past a submerged horizontal cylinder: instantaneous free-surface profile and vorticity distribution for $G / D=5, H / D=0.75$, and $\mathrm{Fr}=0.2,0.3,0.4,0.5$, and 0.6 at $t=200$. Solid and dashed lines represent positive and negative vorticity, respectively. 


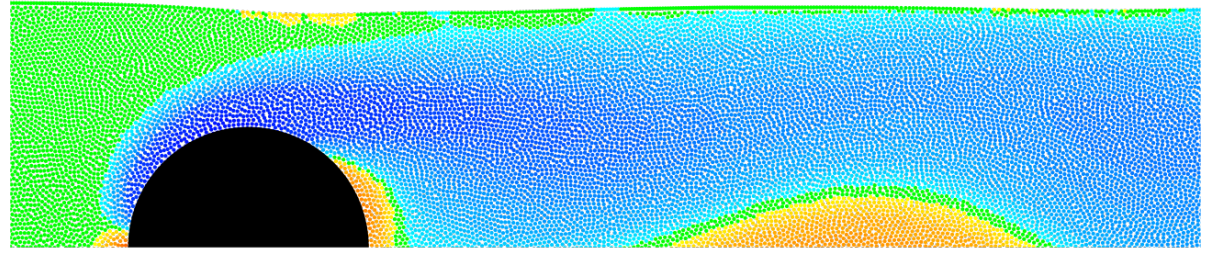

(a) $\mathrm{Fr}=0.2$

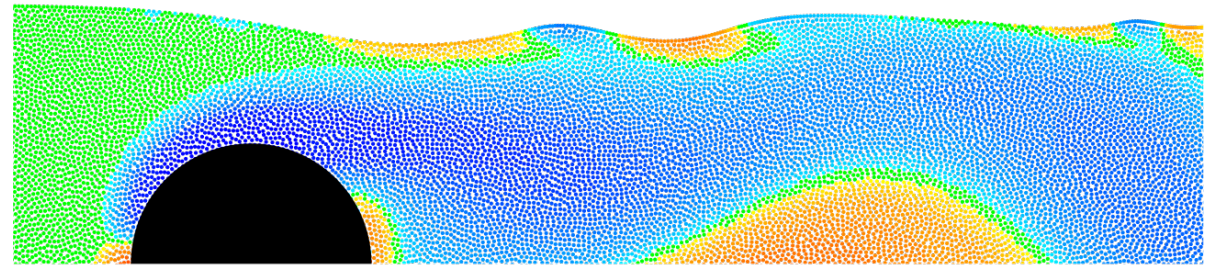

(b) $\mathrm{Fr}=0.3$

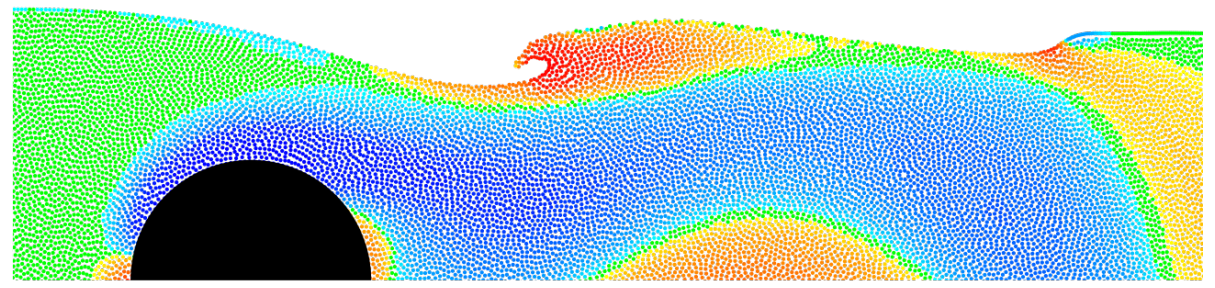

(c) $\mathrm{Fr}=0.4$

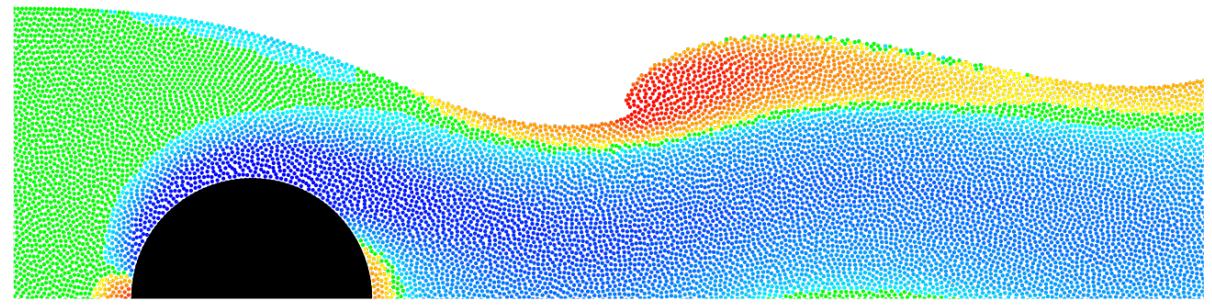

(d) $\mathrm{Fr}=0.5$

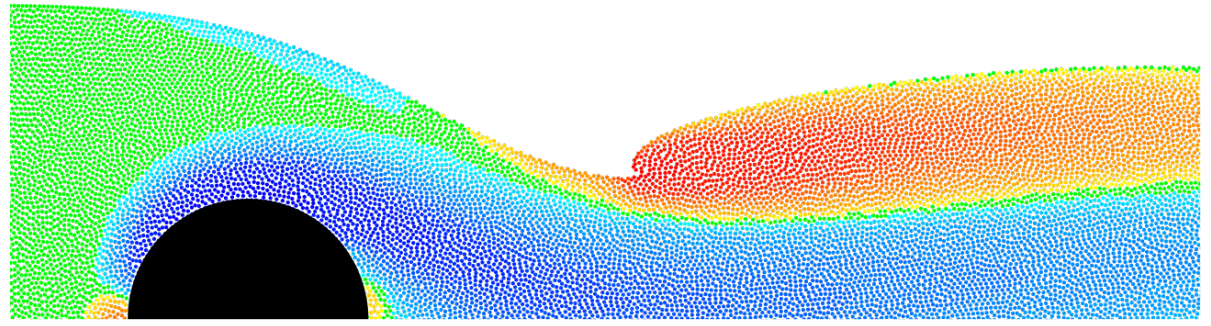

(e) $\mathrm{Fr}=0.6$

Fig. 23 Free-surface flow past a submerged horizontal cylinder: close up view of instantaneous free-surface wave profile and vorticity distribution in the vicinity of the upper part of the cylinder for $G / D=2, H / D=0.5$, and $\mathrm{Fr}=0.2,0.3,0.4,0.5$, and 0.6. Blue represents clockwise negative vorticity; red represents anticlockwise positive vorticity. 


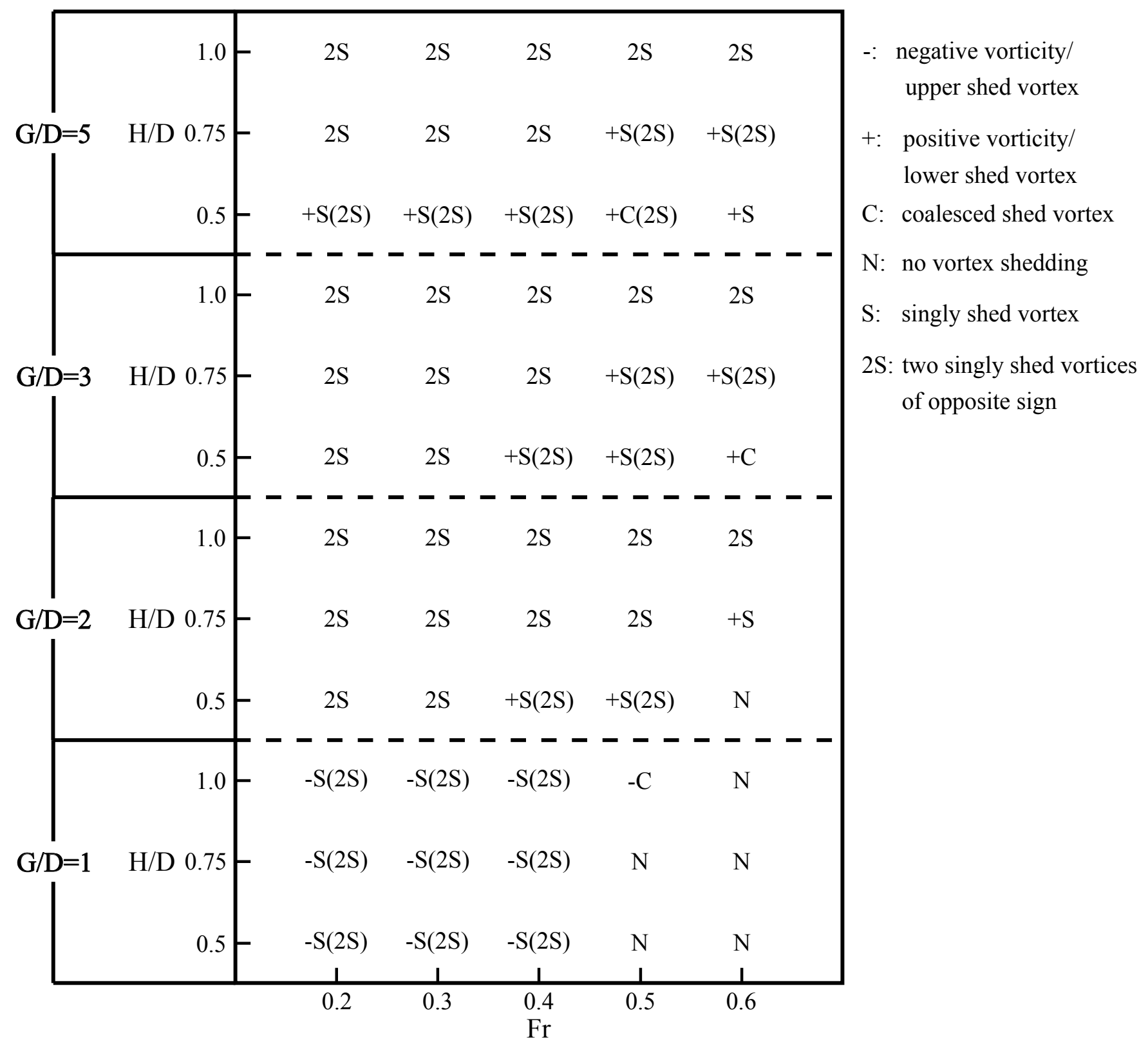

Fig. 24 Map indicating flow patterns obtained for different gap and submergence ratios as the Froude number changes from 0.2 to 0.6 . 


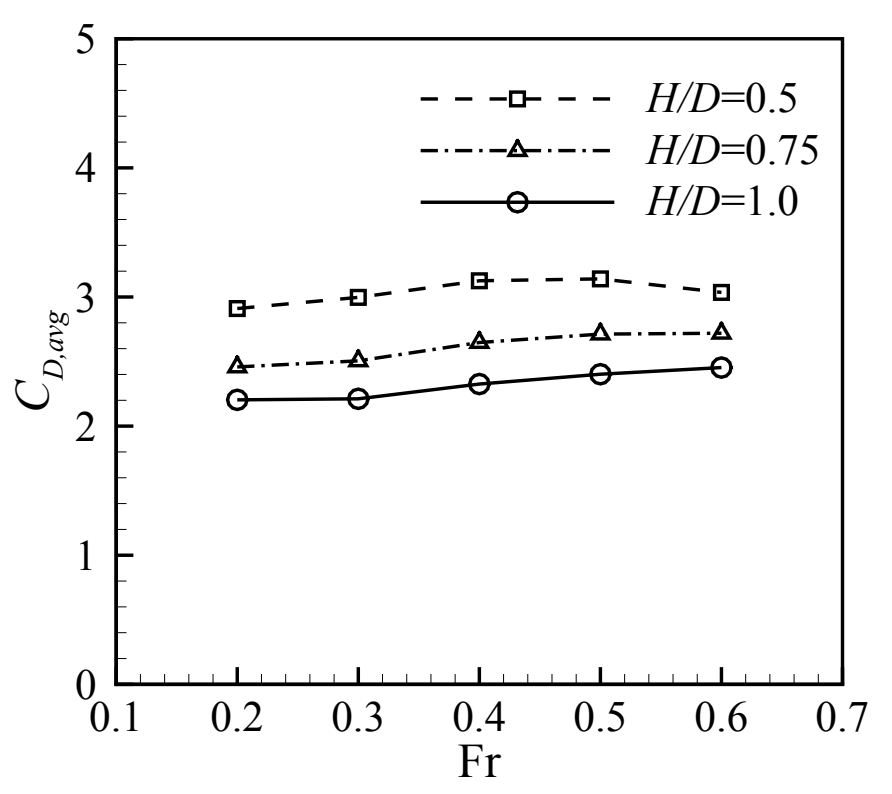

(a) $\mathrm{G} / \mathrm{D}=1$

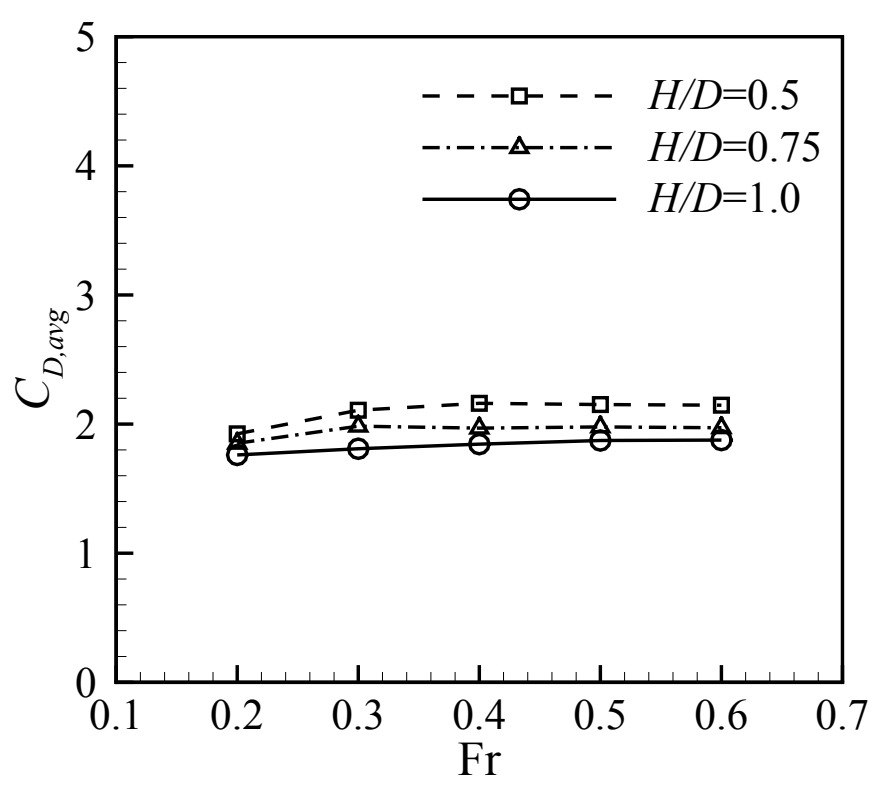

(c) $\mathrm{G} / \mathrm{D}=3$

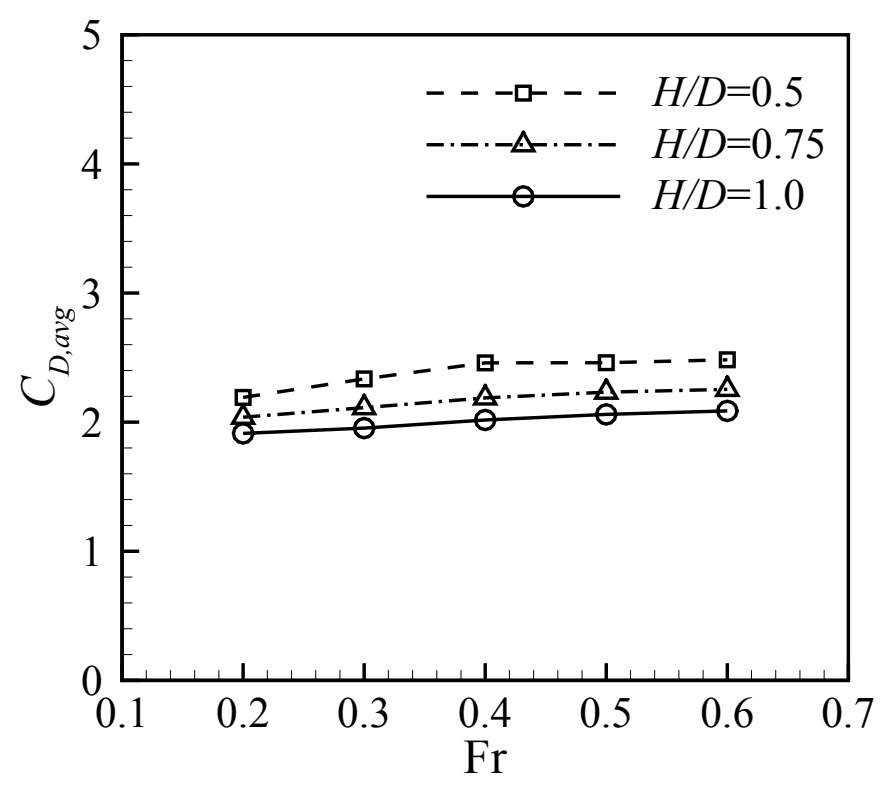

(b) $\mathrm{G} / \mathrm{D}=2$

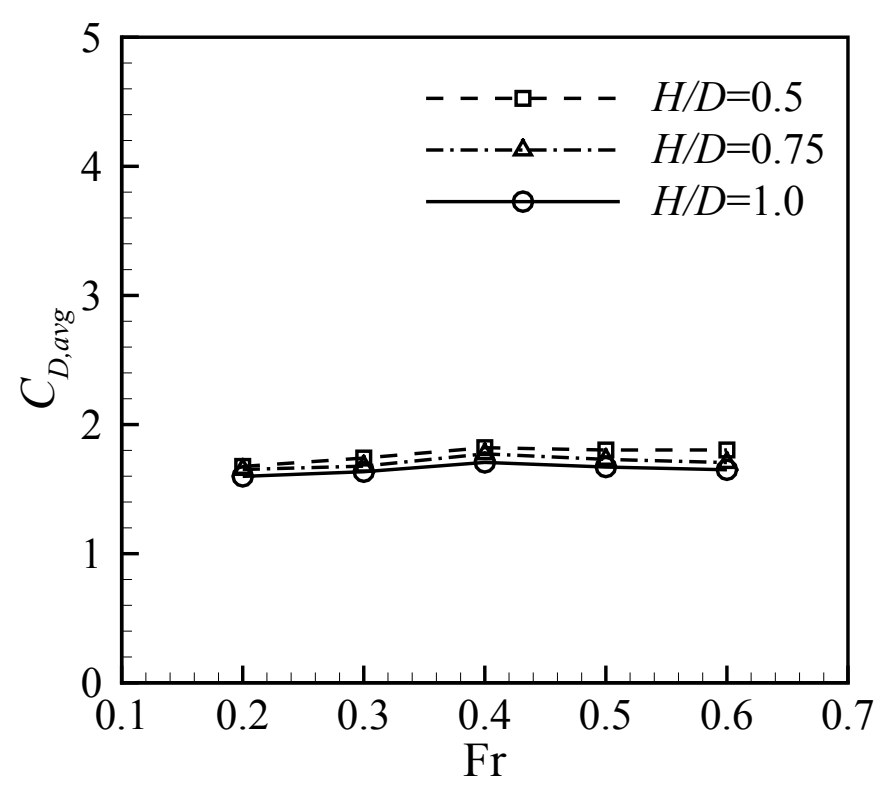

(d) $\mathrm{G} / \mathrm{D}=5$

Fig. 25 Mean drag coefficient as a function of Froude number, obtained for all ranges of submergence and gap ratios considered in the parameter study. 


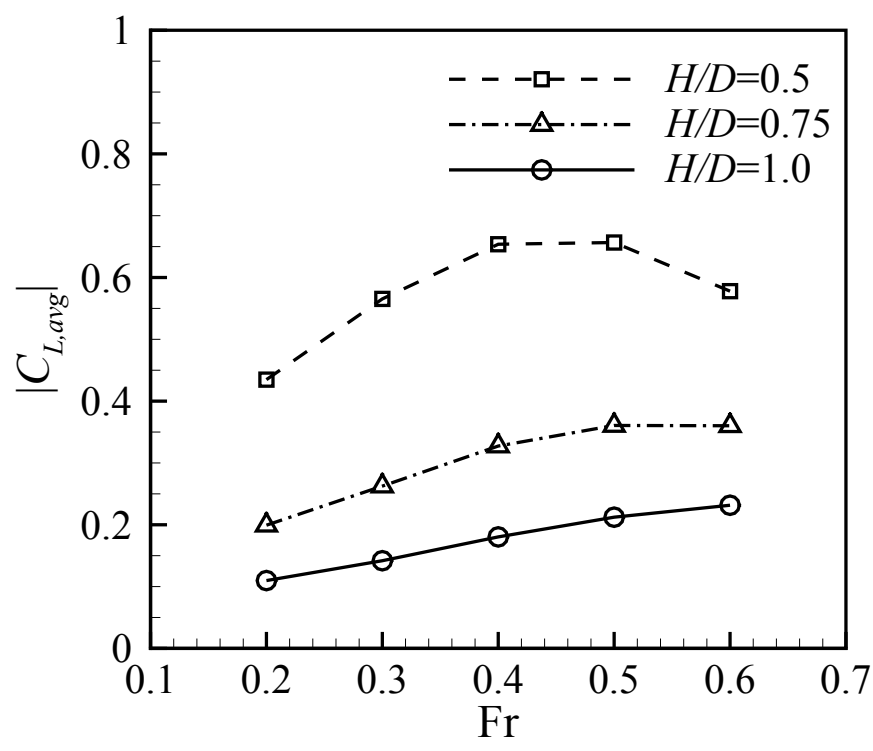

(a) $G / D=1$

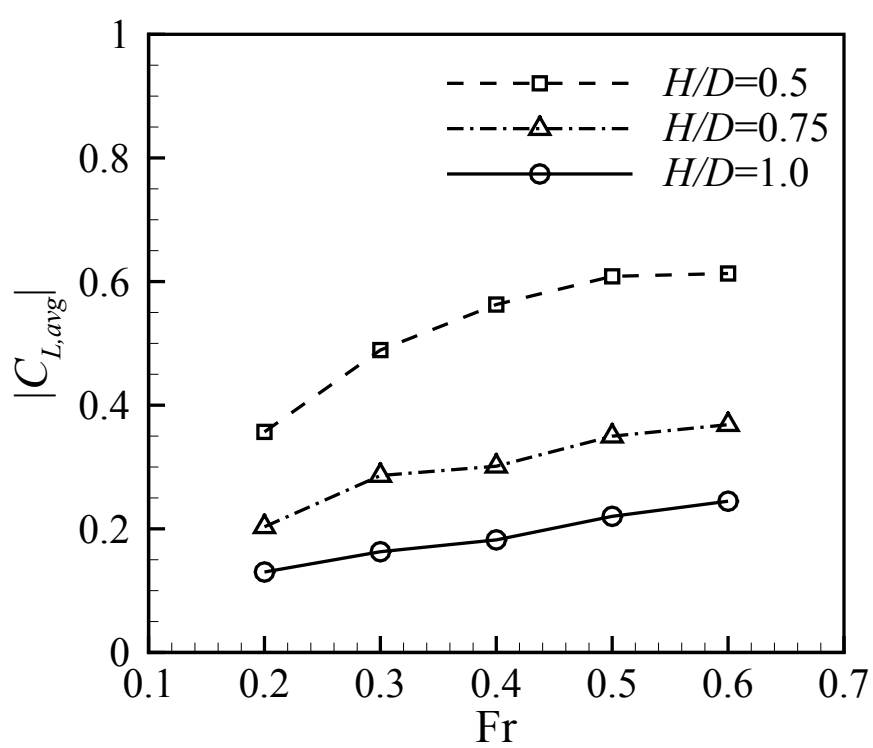

(c) $\mathrm{G} / \mathrm{D}=3$

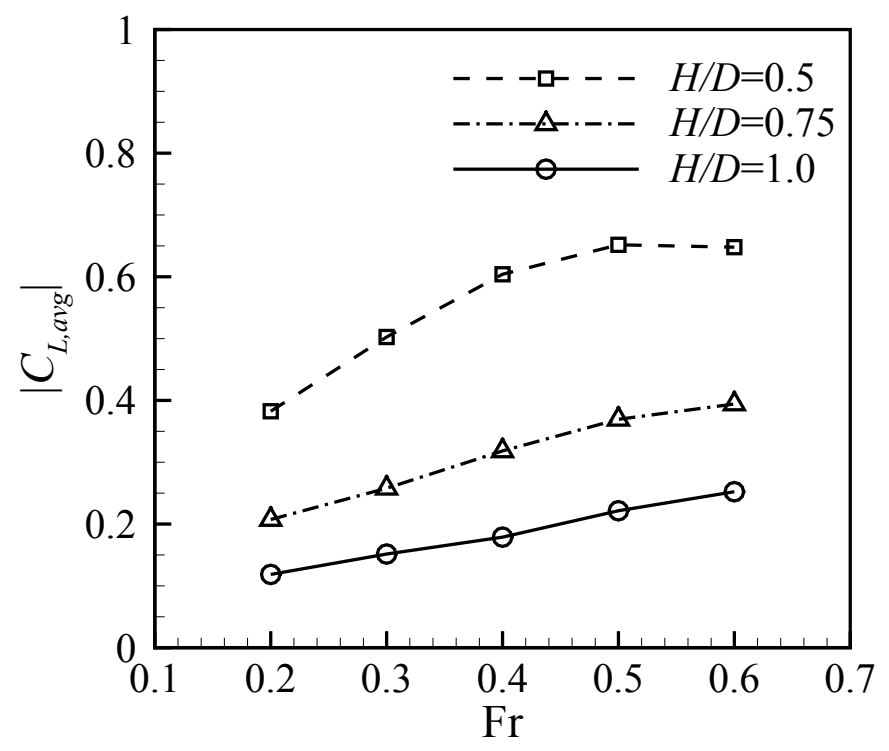

(b) $\mathrm{G} / \mathrm{D}=2$

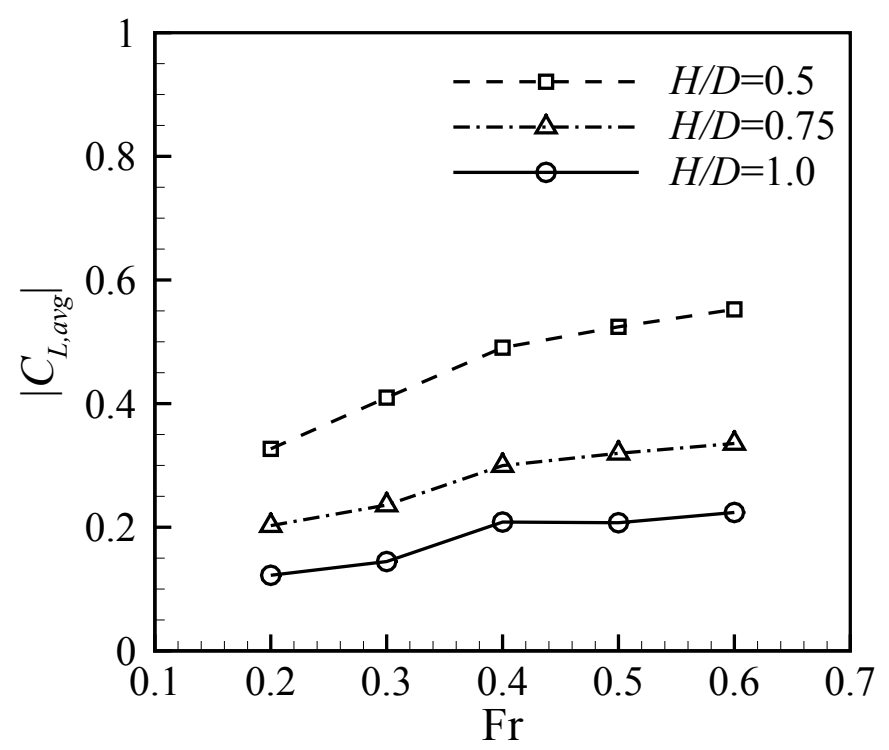

(d) $\mathrm{G} / \mathrm{D}=5$

Fig. 26 Absolute value of mean lift coefficient as a function of Froude number, obtained for all ranges of submergence and gap ratios considered in the parameter study. 


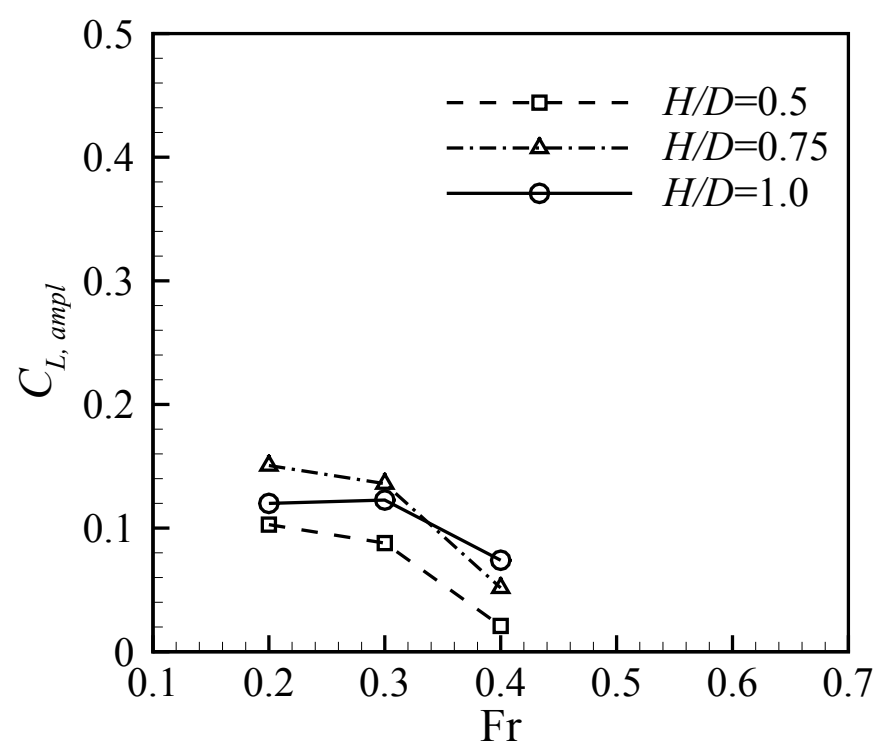

(a) $\mathrm{G} / \mathrm{D}=1$

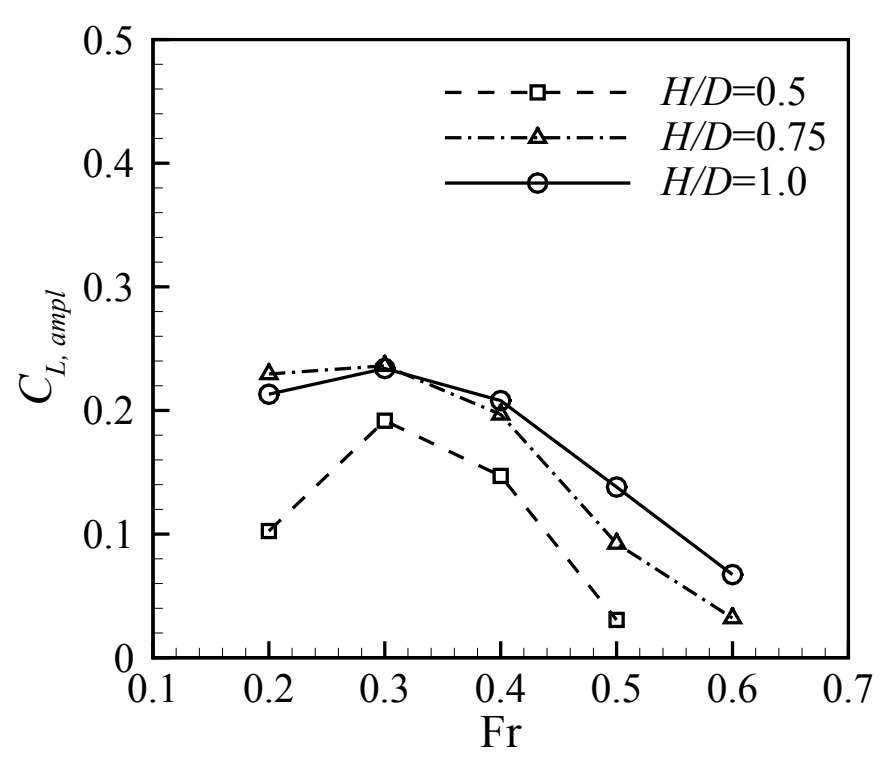

(c) $\mathrm{G} / \mathrm{D}=3$

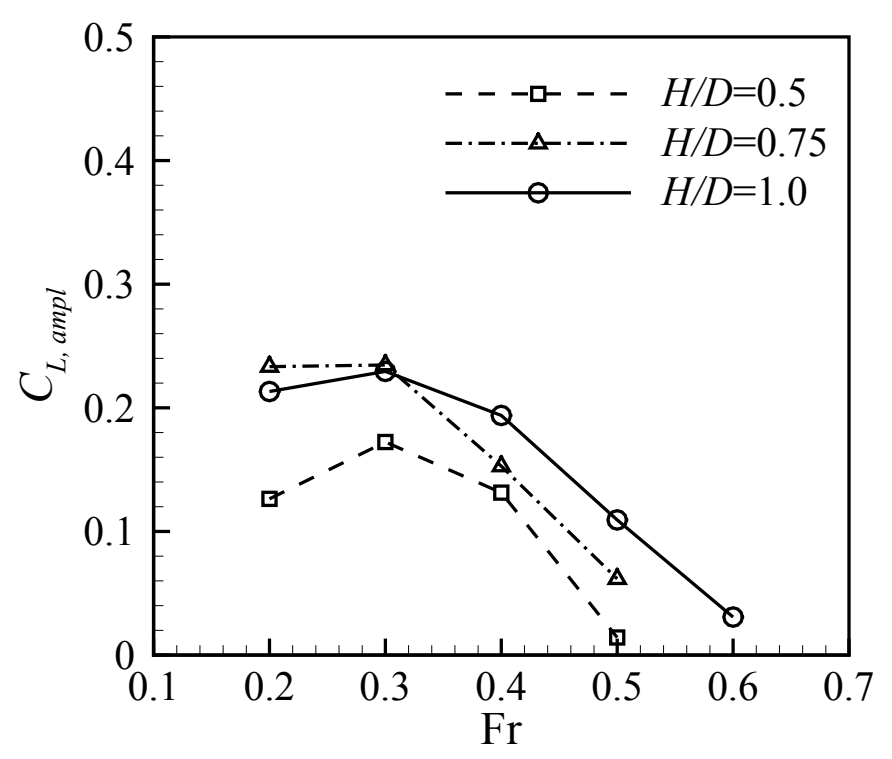

(b) $\mathrm{G} / \mathrm{D}=2$

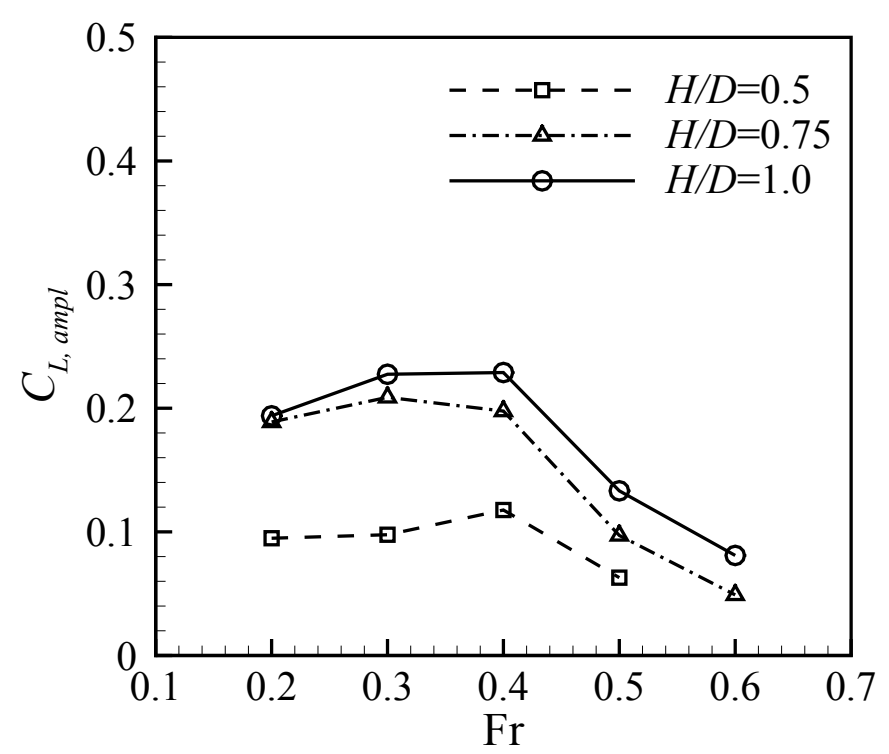

(d) $\mathrm{G} / \mathrm{D}=5$

Fig. 27 Lift oscillation amplitude as a function of Froude number, obtained for all ranges of submergence and gap ratios considered in the parameter study. 


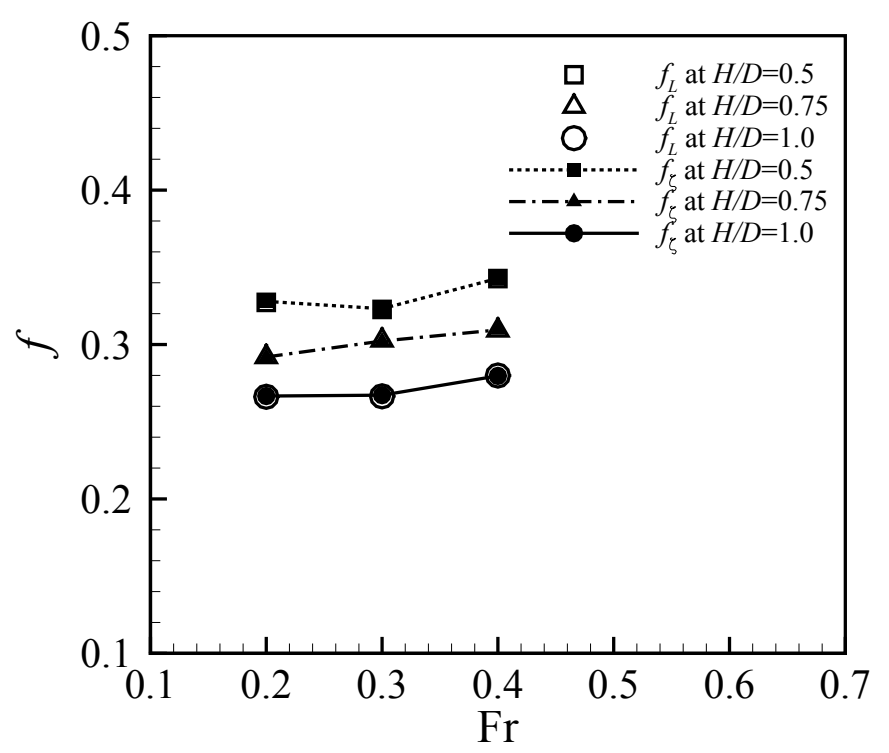

(a) $\mathrm{G} / \mathrm{D}=1$

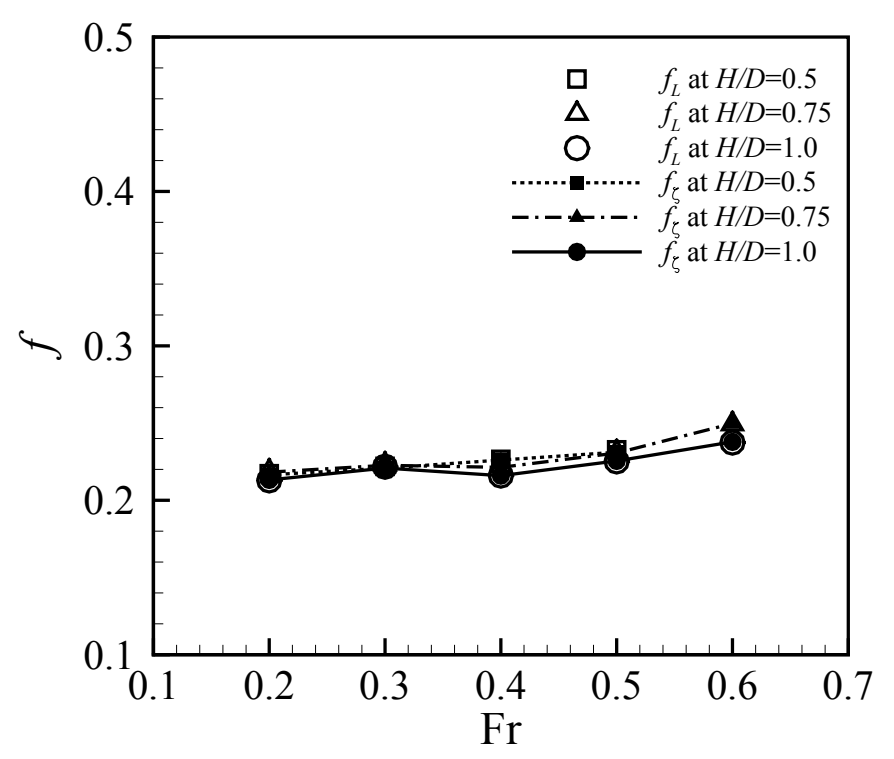

(c) $\mathrm{G} / \mathrm{D}=3$

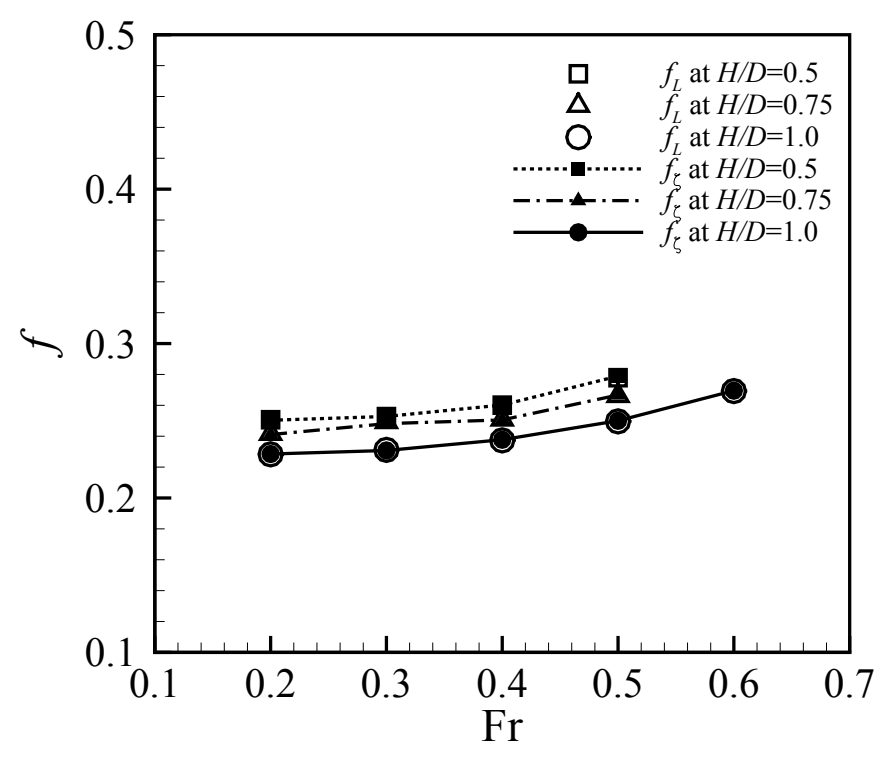

(b) $\mathrm{G} / \mathrm{D}=2$

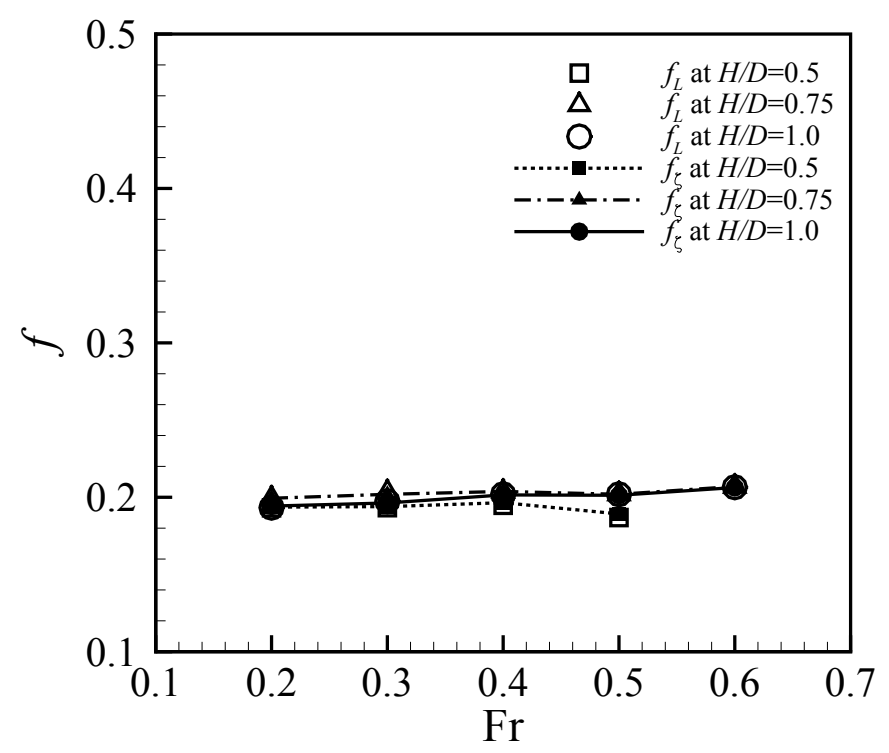

(d) $\mathrm{G} / \mathrm{D}=5$

Fig. 28 Peak frequencies in lift force and free-surface elevation spectra as a function of Froude number, obtained for all ranges of submergence and gap ratios considered in the parameter study. $f_{L}$ and $f_{\xi}$ denote the lift force and free-surface elevation frequencies, respectively. 